\title{
Grassmann Phase Space Methods for Fermions: I. Mode Theory
}

\author{
B. J Dalton ${ }^{1 *}$ J. Jeffers ${ }^{2} \&$ S. M. Barnett ${ }^{3}$ \\ 1. Centre for Quantum and Optical Science, Swinburne University of Technology, \\ Melbourne, Victoria 3122, Australia \\ 2. Dept of Physics, University of Strathclyde, Glasgow G4ONG, UK \\ 3. School of Physics and Astronomy, University of Glasgow, Glasgow G12 8QQ, UK \\ 6th September 2016
}

In both quantum optics and cold atom physics, the behaviour of bosonic photons and atoms is often treated using phase space methods, where mode annihilation and creation operators are represented by c-number phase space variables, with the density operator equivalent to a distribution function of these variables. The anti-commutation rules for fermion annihilation, creation operators suggests the possibility of using anti-commuting Grassmann variables to represent these operators. However, in spite of the seminal work by Cahill and Glauber and a few applications, the use of Grassmann phase space methods in quantum - atom optics to treat fermionic systems is rather rare, though fermion coherent states using Grassmann variables are widely used in particle physics.

The theory of Grassmann phase space methods for fermions based on separate modes is developed, showing how the distribution function is defined and used to determine quantum correlation functions, Fock state populations and coherences via Grassmann phase space integrals, how the Fokker-Planck equations are obtained and then converted into equivalent Ito equations for stochastic Grassmann variables. The fermion distribution function is an even Grassmann function, and is unique. The number of c-number Wiener increments involved is $2 n^{2}$, if there are $n$ modes. The situation is somewhat different to the bosonic c-number case where only $2 n$ Wiener increments are involved, the sign of the drift term in the Ito equation is reversed and the diffusion matrix in the Fokker-Planck equation is anti-symmetric rather than symmetric. The un-normalised $B$ distribution is of particular importance for determining Fock state populations and coherences, and as pointed out by Plimak, Collett and Olsen, the drift vector in its FokkerPlanck equation only depends linearly on the Grassmann variables. Using this key feature we show how the Ito stochastic equations can be solved numerically for finite times in terms of c-number stochastic quantities. Averages of products of Grassmann stochastic variables at the initial

${ }^{*}$ Corresponding Author: Tel: +61039214 8187; fax: +610 392145160 Email address: bdalton@swin.edu.au 
time are also involved, but these are determined from the initial conditions for the quantum state. The detailed approach to the numerics is outlined, showing that (apart from standard issues in such numerics) numerical calculations for Grassmann phase space theories of fermion systems could be carried out without needing to represent Grassmann phase space variables on the computer, and only involving processes using c-numbers. We compare our approach to that of Plimak, Collett and Olsen and show that the two approaches differ.

As a simple test case we apply the $B$ distribution theory and solve the Ito stochastic equations to demonstrate coupling between degenerate Cooper pairs in a four mode fermionic system involving spin conserving interactions between the spin $1 / 2$ fermions, where modes with momenta $-k,+k$ - each associated with spin up, spin down states, are involved. 


\section{Introduction}

This paper presents a phase space theory for many-body fermion systems. There are a range of theoretical treatments of the behaviour of many-body systems in non-relativistic quantum systems, which are found in condensed matter physics, nuclear physics, quantum optics and more recently in the physics of degenerate bosonic and fermionic atomic gases at low temperatures and density. Methods include not only the more traditional Green function [1], variational [2], [3], [4] and stochastic Schrodinger equation-Quantum Monte Carlo [5] approaches, but also phase space methods. In most modern treatments where the many-body systems invove identical particles, a second quantization approach [6] is used, including in this paper. Here the key concept of single particle states (or mode) is introduced, and basis states (Fock states) are defined via the number of particles occupying the various modes. Mode annihilation and creation operators decrease or increase the occupancy by one particle. The annihilation, creation operators satisfy commutation rules (bosons) or anti-commutation rules (fermions). For the fermion systems dealt with in this paper, the occupancy is zero or one, contrasting boson systems where the occupancy is unrestricted.

In phase space theories where the modes are considered separately, the density operator describing the quantum state is represented by a distribution function of phase space variables that are associated with the annihilation and creation operators. Measureable quantities such as quantum correlation functions and Fock state probabilities and coherences are expressed as phase space integrals involving the distribution function and other functions of the phase space variables specific to the measureable quantity. The evolution equation for the density operator is replaced by a Fokker-Planck equation [7] for the distribution function. In turn, the phase space variables are replaced by time dependent stochastic variables, and Ito stochastic equations for these variables are used to give the same results for measured quantities as would be obtained by solving the Fokker-Planck equation. There are several types of distribution function that have been used, notably the $P, Q$ and $W$ functions, associated with the names of Glauber and Sudarshan [8], [9], Husimi [10] and Wigner [11], [12] respectively, and used in conjunction with measureable quantities that involve normally ordered, anti-normally ordered and symmetrically ordered combinations of operators. Phase space theories are often distinguished by whether they employ a single phase space variable and its complex conjugate in association with the mode annihilation and creation operators respectively (single phase space), or whether mode creation and annihilation operators are associated with a pair of independent phase space variables (double phase space). For bosonic systems double phase space theories involving the $P+$ and complex $P$ distributions were first developed by Drummond and Gardiner [16]. Perhaps a more useful distinction between single and double space approaches is whether the distribution function depends on one pair of phase space variables $\left(\alpha, \alpha^{*}\right)$ or $\left(\alpha, \alpha^{+}\right)$- single phase space or on two pairs $\left(\alpha, \alpha^{*}, \alpha^{+}, \alpha^{+*}\right)$ for each mode - double phase space.

For cases involving only a few modes the phase space method has been widely used for bosonic systems, such as photon systems in quantum optics and Bose-Einstein condensates in degenerate quantum gases. Here only a small number of c-number phase space variables are involved either one or two for each of the small nunber of modes. That the phase variables commute mirrors the commutation rules satisfied by the operators. The Fokker-Planck equations for the standard bosonic $P, Q$ and $W$ functions involve derivatives with respect to the independent variables $\alpha$ and $\alpha^{*}$ for each mode. The approach is extensively covered in many text books, such as [13], [14], [15]. The usual approach for few mode fermionic systems - which are restricted to small fermion numbers - is to associate atomic spin operators or pairs of fermion annihilation, creation operators with c-number phase space variables. This approach is described in the textbook [17] and used in the recent Gaussian fermion representation treatment of Corney and 
Drummond [18]- which follows from their previous Gaussian treatment for bosons [19]. However, here the commutation of the phase variables does not mirror the rules for the operators, for which commutation rules more like those for angular momentum operators occur.

This paper presents the basic theory for an alternative phase space distribution function theory for fermions in the separate mode case, based on Grassmann phase space variables. Rather than associating combined pairs of fermion annihilation and creation operators with c-number phase space variables as in the usual approach, the fermion annihilation and creation operators themselves will be directly associated with phase space variables. However, the anti-commutation rules for the operators suggest using phase space variables that mirror these anti-commutation rules. Such phase space variables cannot be c-numbers, but Grassmann numbers are algebraic entities that satisfy anti-commutation rules. Hence in this paper the phase space variables will be Grassmann numbers (g-numbers) and a phase space approach of the $P$ type (or the related un-normalised $B$ distribution) will be used, with independent phase space variables $g$ and $g^{+}$associated with the mode annihilation and creation operators respectively. In the above terminology this would be categorised as a single phase space approach. Grassmann variables have already found application in physics, an early textbook using them was that of Berezin [20] in 1966. More recently, Grassmann variables are widely used in quantum field theory and particle physics, see for example [21]. However, there are few treatments of phase space theories based on Grassmann variables and their applications in quantum-atom optics. An early work on the subject was that of Cahill and Glauber [22], based around fermion coherent states and the standard $P, Q$ and $W$ distributions. Applications to problems such as interacting Fermi gases have been presented by Plimak, Collett and Olsen [23], who introduced the key idea of using the un-normalised $B$ distribution. These two papers involved phase space variables $g$ and $g^{*}$ associated with the mode annihilation and creation operators (which can also be regarded as independent phase space variables). More recently, the present authors [24] have shown that Grassmann phase space theory provides a valid treatment of the Jaynes-Cummings model. Other work on Grassman phase space theory is presented in [25], [26], [27] and [28].

The importance of two of these previous papers involving Grassmann phase space methods for fermions deserves special recognition. The seminal paper of Cahill and Glauber [22] first introduced fermion coherent states based on Grassmann variables and showed how the density operator could be represented in a phase space theory approach involving Grassmann variables. However, this paper did not treat dynamical evolution problems - either time evolution under a Liouville-von Neumann or master equation or temperature evolution under a Matsubara equation. Thermal evolution (imaginary time dynamics) in interacting Fermi gases was treated in the key paper by Plimak, Collett and Olsen [23], again using a phase space approach, but now based on the un-normalised $B$ distribution - which they recognised as a more suitable basis for numerical work. Fokker-Planck and Ito stochastic equations were employed in their work and numerical studies were also carried out. Their approach could also be extended to treat real-time dynamics. They recognised that the $B$ distribution had the important feature of leading to linear transformations of stochastic Grassmann variables when evolution occurs. In the present paper we also treat evolution probems via a phase space approach with two independent variables $g$ and $g^{+}$associated with each mode, again using an un-normalised $B$ distribution function based on Bargmann coherent states. Our focus is on real-time dynamics. However, although using the $B$ distribution first applied in Ref [23], our theoretical approach is different to that of Plimak, Collett and Olsen in terms of the basic principle used for deriving the important Ito stochastic equations that are needed for numerical work. Consequently, the equations we obtain are different to those based on the approach in Ref. [23]. We believe that our approach is logical and internally consistent. In Section 7 we compare our approach to that of Ref. [23], but as technical arguements based on details to be presented below are involved, 
this Section is placed after the main part of the paper.

The formal theory set out in this paper is aimed at introducing the distribution in a correct but simple fashion, starting from the canonical representation of the density operator in terms of Bargmann states for fermions, which are defined in terms of fermion creation operators and Grassmann variables for the modes. The canonical form of the density operator involves a Grassmann distribution function that depends on two independent phase space variables $g$ and $g^{+}$for each mode, these being related to the annihilation and creation operators, respectively. We consider Grassmann distribution functions of the normalised $P$ and un-normalised $B$ type, though focusing more on the $B$ distribution because of its greater utility for numerical work. These are both even Grassmann functions, and we show they are unique. We establish the key expressions for measureable quantities such as quantum correlation functions in terms of phase space integrals over the $g$ and $g^{+}$involving the distribution functions. The derivation of the correspondence rules and the Fokker-Planck equation is carefully spelt out, and here the Fokker-Planck equation involves Grassmann derivatives with respect to phase space variables $g$ and $g^{+}$. For the thermal evolution considered in Ref [23], only Grassmann derivatives with respect to $g$ are involved. We find that in the fermion case the diffusion matrix is found to be anti-symmetric - rather than symmetric as in the boson case. Detailed expressions for the drift vector and diffusion matrix are presented for both $P$ and $B$ cases, the latter having drift terms that depend linearly on the Grassmann phase space variables. The drift, diffusion terms are odd, even Grassmann functions respectively. Furthermore, we provide a detailed proof that Ito stochastic equations which are equivalent to the Fokker-Planck equation can be obtained. We find that the relationship between the classical term in the Ito equations and the drift term in the Fokker-Planck equation has the reversed sign to that for the boson case, whilst the noise term is related to the diffusion matrix in the same way as in the boson case, in spite of the diffusion matrix being anti-symmetric in the fermion case. However, the number of c-number Wiener increments involved is $2 n^{2}$, if there are $n$ modes. The situation is somewhat different to the bosonic c-number case where only $2 n$ Wiener increments are involved. Finally, we demonstrate the important general result for the $B$ distribution case - also occuring in Plimak et al [23] - that the stochastic Grassmann phase space variables at later times are related linearly to these variables at an earlier time and where the linear relationships only involve c-numbers and not Grassmann variables. Although these c-numbers involve Wiener increments we have justified the important conclusion that numerical calculations for measureable quantities involving stochastic Grassmann phase space variables can be carried out, only the stochastic averages of products of the initial Grassmann phase space variables being required - with these being obtained from the initial conditions. This resolves an important issue regarding the applicability of Grassmann phase space theories for numerical calculations. Our approach to applying the theory numerically is set out in some detail in Section 5.9. The impracticability of representing Grassmann variables on a computer was actually thought to preclude such numerical work [29]. To quote their words "Representations for fermionic density operators were introduced by Cahill and Glauber using fermionic coherent states. These provide a means of defining quasiprobabilities for fermionic states analogous to the well-known bosonic distributions. However, the resulting quasiprobabilities are functions of noncommuting Grassmann variables and are thus not directly computationally accessible. Nevertheless, fermion coherent states and Grassmann algebra are useful for deriving analytical results in Fermi systems.". There are of course many other key issues such as convergence, sampling error etc that also must be dealt with in applying our work in actual numerical calculations. We do not claim to have dealt with these issues in the present paper.

In phase space theories where fields rather than separate modes are considered, the density operator describing the quantum state is represented by a distribution functional involving 
phase space field functions that are associated with the field annihilation and creation operators. This extension is important even for bosonic cases where sometimes large numbers of modes that have non-zero occupancy are involved, such as for spatial squeezing in quantum optics [30]. Measureable quantities such as quantum correlation functions and Fock state probabilities and coherences are expressed as phase space functional integrals involving the distribution functional and other functions of the phase space fields specific to the measureable quantity. The evolution equation for the density operator is replaced by a functional Fokker-Planck equation for the distribution functional. In turn, the phase space fields are replaced by time dependent stochastic fields, and Ito stochastic field equations for these stochastic fields are used to give the same results for measured quantities as would be obtained by solving the functional Fokker-Planck equation. Such phase space distribution functional theories have been widely used in the bosonic case - such as for photons [30], where the field functions are c-number fields. The paper by Steele et al [31] sets out the theory for bosonic gases, and there have been many further developments such as including gauge fields [32], hybrid theories [33], [34], [35], [36] involving $P$ and $W$ distributions and double phase spaces, the last allowing for time dependent mode functions. The usual approach for fermionic systems was based on atomic spin field operators [37] or pairs of fermion field operators, these being associated with c-number field functions. However, for analogous reasons to the few mode case, the present paper involves associating the fermion field annihilation and creation operators directly with field functions. Again, the anti-commutation rules satisfied by the fermion field operators suggest using field functions with similar anti-commutation properties and hence the present theory will employ Grassmann fields rather than c-number fields. Grassmann fields can be expanded in terms of c-number mode functions, but now the expansion coefficients are Grassmann variables. This Grassmann field approach was adopted for fermion problems by Plimak, Collett and Olsen [23].

We have also developed the basic theory for an alternative phase space distribution functional theory for fermions when large numbers of modes are involved, based on Grassmann fields. This theory will be presented in a forthcoming paper [38]. The theory involved two independent phase space field functions $\psi$ and $\psi^{+}$which are associated with the field annihilation and creation operators respectively. This extension is of key importance in the common situation where large numbers of fermions are involved, since the Pauli exclusion principle guarantees that large numbers of modes are needed to accommodate them. It is then preferable to frame the theory around field annihilation and creation operators rather than separate mode operators, though we have demonstrated the two approaches are interchangeable via mode expansions for the Grassmann fields. After associating the field annihilation and creation operators with Grassmann field functions rather than modal Grassmann phase space variables, we introduced the Grassmann distribution functionals that replace the density opeator starting from the distribution functions. Both $P$ and $B$ distribution functionals have been considered. We then derived the expressions for measurable quantities such as quantum correlation functions, Fock state probabilities and coherences as Grassmann phase space functional integrals involving the distribution functional and field functions specific to the measureable quantity. A careful derivation has been given of the functional Fokker-Planck equation for the distribution functional starting from the Fokker-Planck equation, with expressions for the drift and diffusion terms in the functional Fokker-Planck equations related to the corresponding terms therein. The correspondence rules for the field annihilation and creation operators have also been established via mode expansions, but which then can be used to derive functional Fokker-Planck equations directly from the evolution equation for the density operator when the Hamiltonian is expressed in terms of field operators. After defining the time dependent stochastic Grassmann fields that replace the field functions, we then derived the Ito stochastic field equations, giving explicit expressions for the classical and noise fields in terms of the related quantities in the Ito stochastic 
equations. An important result was establishing the general relationship between the classical and noise field terms in the Ito stochastic field equations and the drift and diffusion terms in the functional Fokker-Planck equation. This relationship was then used to determine the classical and noise terms in the Ito stochastic field equations directly from the functional Fokker-Planck equation without requiring mode expansions. For the $B$ distribution case - also occuring in Plimak et al [23] - we showed that the stochastic Grassmann fields at later times are related linearly to these fields at an earlier time, and where the linear relationships only involve (stochastic) c-numbers and not Grassmann fields, thereby allowing numerical calculations based on stochastic Grassmann fields to be carried out. However, there are other important issues involved in carrying out numerical calculations for Grassmann fields - for example the position dependence of the stochastic Grassmann fields must be represented as points on a spatial grid, and in some cases non-local Ito stochastic field equations must be solved.

A much more detailed account of Grassmann phase space theory for fermions, covering both the separate mode and the field cases and the relationship to c-number phase space theory for bosons is set out in the recently published book by the present authors entitled Phase Space Methods for Degenerate Quantum Gases [39]. Readers are refered to this textbook for a more complete treatment. Here we focus only on presenting the most essential results.

The plan of this paper is as follows. The approach adopted is to focus on the phase space theory aspects in the main body of the paper, with the underlying maths and detailed derivations set out in the Appendices. In Section 2 the basic second quantisation concepts such as mode creation and annihilation operators, Fock states, super-selection rules involved in treating systems of identical fermionic atoms are outlined. Phase space theory for separate mode systems is described in Section 3, focusing on distribution functions of the normalised $P$ and un-normalised $B$ type in phase spaces and results for quantum correlation functions, Fock state populations and coherences as phase space integrals. Section 4 presents the Fokker-Planck equations that follow from the master equation for the density operator by applying the correspondence rules associated with mode operators, with detailed forms for the drift vector and diffusion matrix set out for the case of the $B$ distribution. The following Section 5 sets out the Ito stochastic equations that are equivalent to the Fokker-Planck equation, demonstrating how the classical and noise terms are related to its drift and diffusion terms and proving for the first time the key result that the equation for the noise term can be solved. This section, which focuses on the $B$ distribution case, also demonstrates that the stochastic phase variables at a later time are linearly related to those at an earlier time via stochastic coefficients which are just c-numbers, thereby showing that stochastic calculations involving a Grassmann phase space theory can still be carried out without having to represent Grassmann variables on the computer, as Plimak et al [23] had stated. The approach to applying the theory numerically is set out in some detail in SubSection 5.9. An analytic application of the separate mode theory for fermions is presented in Section 6, where a four mode system involving Cooper pairs for interacting spin $1 / 2$ atoms is studied. A comparison of our theoretical approach to that of Ref [23] is made in Section 7. A summary of the paper and the key results obtained follows in Section 8.

Online supplementary material and a website version of this paper [RefNumber] contains the Appendices which cover both the basic maths and details for the derivations of results in this paper which are too lengthy to present in the journal version. The key features of Grassmann algebra and calculus are set out in Appendix A. In Appendix B fermion coherent states and their main properties are outlined. The derivation of the canonical form for the density operator in terms of Bargmann state projectors is set out in Appendix C. Phase space integral expressions for quantum correlation functions, Fock state populations and coherences are derived in Appendix D. The derivation of the correspondence rules for fermion Fokker-Planck equations is presented in Appendix E for the $B$ distribution case and the analogous rules and 
Fokker-Planck equations for the $P$ distribution case are recorded in Appendix F. The derivation of the time dependence of stochastic averages of Grassmann functions is carried out in Appendix G. Details of the comparison with the approach of Plimak et al are presented in Appendix H.

\section{Systems of Identical Fermions}

\subsection{Modes, Annihilation and Creation Operators, Fock States.}

In this paper we consider system of identical fermions, which in quantum-atom optics could be fermionic atoms or molecules. The fermions will be associated with position and momentum operators describing their centre of mass motion, but also may exist in different internal states such as those with differing hyperfine quantum numbers. The approach used here is that of second quantisation (see for example [1], [6]), which is based on the existence of modes (or single particle states) $\left|\phi_{i}\right\rangle$ - listed in a prescribed order $1,2, \cdots, i, \cdots, n$ - which the identical fermions may occupy. Modes associated with different internal states $|\alpha\rangle$ may be designated $\left|\phi_{\alpha i}\right\rangle=\left|\phi_{i}^{(\alpha)}\right\rangle|\alpha\rangle$ in which $\left|\phi_{i}^{(\alpha)}\right\rangle$ describes the centre of mass motion, but for the moment we will ignore the internal state since experiments can be carried out with all fermions in the same internal state. The position representation of the mode $\left|\phi_{i}\right\rangle$ is $\phi_{i}(\mathbf{r})=\left\langle\mathbf{r} \mid \phi_{i}\right\rangle$ are is referred to as the mode function. The modes are chosen to be orthonormal and complete.

$$
\begin{aligned}
\int d \mathbf{r} \phi_{i}^{*}(\mathbf{r}) \phi_{j}(\mathbf{r}) & =\delta_{i ; j} \quad\left\langle\phi_{i} \mid \phi_{j}\right\rangle=\delta_{i ; j} \\
\sum_{i} \phi_{i}^{*}(\mathbf{r}) \phi_{i}\left(\mathbf{r}^{\prime}\right) & =\delta\left(\mathbf{r}-\mathbf{r}^{\prime}\right) \quad \sum_{i}\left|\phi_{i}\right\rangle\left\langle\phi_{i}\right|=\hat{1}
\end{aligned}
$$

The Pauli exclusion principle precludes the existence of multi-fermion states where more than one fermion occupies any particular mode. Multi-fermion Fock states (which are represented in first quantisation as anti-symmetrised products of the occupied single particle state) can be specified by just stating the number of fermions $\nu_{i}=0,1$ that occupy each mode $\left|\phi_{i}\right\rangle$. Fock states can be written in second quantisation form by introducing fermion creation and annihilation operators. These are denoted $\hat{c}_{i}^{\dagger}, \hat{c}_{i}$ and satisfy the anticommutation rules

$$
\begin{aligned}
& \left\{\hat{c}_{i}, \hat{c}_{j}^{\dagger}\right\} \equiv \hat{c}_{i} \hat{c}_{j}^{\dagger}+\hat{c}_{j}^{\dagger} \hat{c}_{i}=\delta_{i j}, \\
& \left\{\hat{c}_{i}, \hat{c}\right\}=0=\left\{\hat{c}_{i}^{\dagger}, \hat{c}_{j}^{\dagger}\right\} .
\end{aligned}
$$

We note that $\hat{c}_{i}^{2}=\left(\hat{c}_{i}^{\dagger}\right)^{2}=\hat{0}$ as may also be seen from the anti-commutation rules. This similarity to the Grassmann variables is the reason why Grassmann phase space variables are chosen for fermions. The Fock states in second quantisation form are

$$
|\nu\rangle=\left(\hat{c}_{\phi_{1}}^{\dagger}\right)^{\nu_{1}}\left(\hat{c}_{\phi_{2}}^{\dagger}\right)^{\nu_{2}} \cdots\left(\hat{c}_{\phi_{n}}^{\dagger}\right)^{\nu_{n}}|0\rangle=\left|\Phi_{\{\nu\}}\right\rangle
$$

where the occupancy notation $\nu \equiv\left\{\nu_{1}, \nu_{2}, \cdots, \nu_{i}, \cdots \nu_{n}\right\}$ includes all the modes. The vacuum state $|0\rangle$ is an extra state introduced in second quantisation and represents the situation in which no fermions are present. The convention in (4) is that the $\hat{c}_{\phi_{i}}^{\dagger}$ are arranged in order with $\hat{c}_{\phi_{1}}^{\dagger}$ on the left and $\hat{c}_{\phi_{n}}^{\dagger}$ on the right, and where $\left(\hat{c}_{\phi_{i}}^{\dagger}\right)^{0}=\hat{1},\left(\hat{c}_{\phi_{i}}^{\dagger}\right)^{1}=\hat{c}_{\phi_{i}}^{\dagger}$. The modes are listed in the prescribed order to avoid duplication of the Fock states. The Fock states are orthogonal and normalised

$$
\langle\nu \mid \xi\rangle=\delta_{\nu_{1} \xi_{1}} \cdots \delta_{\nu_{n} \xi_{n}}
$$


and are eigenstates of the fermion number operator $\hat{N}=\sum_{i} \hat{n}_{i}$, where $\hat{n}_{i}=\hat{c}_{i}^{\dagger} \hat{c}_{i}$ is the number operator for mode $\left|\phi_{i}\right\rangle$. It is easy to show from the anticommutation rules that the eigenvalues for the fermionic $\hat{n}_{i}$ are restricted to 0,1 . We have

$$
\begin{aligned}
\hat{N}|\nu\rangle & =N|\nu\rangle \\
N & =\sum_{i} \nu_{i}
\end{aligned}
$$

The Fock states form a basis for a Hilbert space, as we will see.

From the anticommuation rules we can then see that

$$
\begin{aligned}
\hat{c}_{i}\left|\nu_{1} ; \cdots ; 0 ; \cdots, \nu_{n}\right\rangle & =0 \\
\hat{c}_{i}\left|\nu_{1} ; \cdots ; 1 ; \cdots, \nu_{n}\right\rangle & =(-1)^{\eta_{i}}\left|\nu_{1} ; \cdots ; 0 ; \cdots, \nu_{n}\right\rangle
\end{aligned}
$$

where $(-1)^{\eta_{i}}=+1$ or -1 according to whether there are an even or odd number of modes listed preceding the mode $\left|\phi_{i}\right\rangle$ which are occupied $\left(\eta_{i}=\sum_{j<i} \nu_{j}\right)$.

\subsection{Quantum States and Super-selection Rules}

The general physical pure state $|\Psi\rangle$ for $N$ identical fermions can be written as a quantum superposition of the basis Fock states.

$$
\begin{aligned}
|\Phi\rangle_{n} & =\sum_{\nu_{1} \cdots \nu_{n}} B_{n}(\nu)|\nu\rangle \\
\sum_{\nu}\left|B_{n}(\nu)\right|^{2} & =1
\end{aligned}
$$

where the sum runs over the occupancy numbers $\nu \equiv\left\{\nu_{1} \cdots \nu_{n}\right\}$, the $B_{n}(\nu)$ are complex coefficients and where $\sum_{i=1}^{n} \nu_{i}=N$. Note that the sum is only over occupation numbers, subject to the constraint that the total occupancy is $N$. The last equation ensures that the state is normalised. If we consider all the states $|\nu\rangle$ arranged in order of total occupancy $\sum_{i} \nu_{i}=0,1,2,3 \cdots$ a heirarchy of basis states for identical particle systems with various total particle numbers $N=0,1,2 \cdots$ can be listed. It is then convenient to mathematically define a Hilbert space (Fock space) describing identical fermion systems with all possible total particle numbers $N$ containing all superpositions of the form

$$
|\Phi\rangle=\sum_{n} C_{n}|\Phi\rangle_{n}=\sum_{\nu_{1} \cdots \nu_{n}} B(\nu)|\nu\rangle
$$

where the $C_{n}$ are complex coefficients. In the last expression the restriction $\sum_{i=1}^{n} \nu_{i}=N$ does not apply to the $B(\nu)$. Such a state $|\Phi\rangle$ is not physical, as super-selection rules (SSR) [40] do not allow superpositions of states with differing total particle numbers (see [41] and references therein for recent discussions on SSR). However states of this form are useful mathematically, even though the pure physical states are restricted to subspaces of this Hilbert space. An example of non-physical states are the fermion coherent states (see Appendix 11), where not only are there a linear combinations of Fock states with differing fermion number, but also the expansion coefficients are Grassmann numbers instead of c-numbers.

However, the physical states for identical particle systems are not restricted to pure states of the form (9). Even when the number of particles $N$ is prescribed, for closed systems there are mixed states described by a quantum density operator $\hat{\rho}_{n}$ of the form

$$
\hat{\rho}_{n}=\sum_{\nu} \sum_{\xi} \rho_{n}(\nu, \xi)|\nu\rangle\langle\xi|
$$


rather than a state vector. The complex coefficients $\rho_{n}(\nu, \xi)$ are the density matrix elements. The requirements that the density operator is hermitean, $\hat{\rho}_{n}=\hat{\rho}_{n}^{\dagger}$, has unit trace $\operatorname{Tr}\left(\hat{\rho}_{n}\right)=1$, and for a mixed state satisfies the condition $\operatorname{Tr}\left(\hat{\rho}_{n}^{2}\right)<1$ lead to well-known constraints on the density matrix elements. For pure states we can write $\hat{\rho}_{n}=|\Phi\rangle_{n}\left\langle\left.\Phi\right|_{n}\right.$ and $\operatorname{Tr} \hat{\rho}_{n}^{2}=1$ and in this case the density matrix elements are $\rho_{n}(\nu, \xi)=B_{n}(\nu) B_{n}^{*}(\xi)$.

For open systems (which particles can enter or leave), physical states can be prepared in which the number of identical fermions is not prescribed. A generalisation of the system state is required to incorporate such cases where the system composition is indefinite. Such states are mixed and also described by a density operator $\hat{\rho}$. However, super-selection rules require that the density operator can only be of the form

$$
\hat{\rho}=\sum_{n} f_{n} \hat{\rho}_{n}
$$

where $\sum_{n} f_{n}=1, f_{n} \geq 0$, which only involves component density operators $\hat{\rho}_{n}$ for systems with specified total fermion numbers $N$, each weighted by real, positive $f_{n}$. The density operator satisfies the earlier requirements for mixed states. Pure states have only one $f_{n}=1$ and all others vanish. More general density operators such as

$$
\hat{\rho}=\sum_{n} \sum_{M} \rho_{N, M}|\Phi\rangle_{n}\left\langle\left.\Phi\right|_{M}\right.
$$

with non-zero $\rho_{N, M}$ do not represent physical states, even though they are mathematical operators in the general mathematical Hilbert space. The physically allowed density operators both for pure or mixed states all commute with the number operator

$$
[\hat{N}, \hat{\rho}]=0
$$

As we will see, the super-selection rule leads to restrictions on the phase space distribution function, so a brief discussion of its origin is appropriate. One basic justification of the SSR is that in the non-relativistic quantum physics of many atom systems that we are considering, there are no available processes that could prepare a SSR non-compliant state. The Hamiltonian commutes with the total particle number and hence if $|\Phi\rangle$ in Eq.(11) is a solution of the Schrodinger equation it follows that the $\left|C_{n}\right|^{2}$ are time independent. Hence the quantum superposition - if it existed as a physical state- would need to have been prepared at the initial time. Such a superposition is of states whose energies differ by relativistic energies of order the rest mass energy $m c^{2}$ - the single atom and atom-atom interaction energies are non-relativistic and there are no non-relativistic processes available that could create such a state. For example, processes such as the dissociation of up to $n$ diatomic molecules into pairs of fermionic atoms can be described via entangled molecule-atom states. However the state for the fermionic atoms alone will be a mixed state described by a (reduced) density operator which is diagonal in fermion numbers - these ranging from 0 up to $2 n$. Furthermore, even if such a superposition as in Eq.(11) existed a second justification for invoking the SSR is that there is no observable that could measure the phase difference between states with differing total fermion numbers - this phase difference would be associated with a relativistic frequency, and this phase difference is needed if the SSR non-compliant state is to be specified. Only measurements on total fermion number are feasible - which for a pure state would be proportional to $\left|C_{n}\right|^{2}$. A third justication is that all the measurements on multi-fermion states can be interpreted without requiring SSR non-complying states to exist. The situation is mirrored in the boson case where as Leggett [42] has pointed out (see also Bach et al [43], Dalton and Ghanbari [44]), a highly occupied number state for a single mode with $N$ bosons has coherence properties of high order $n$, as long as $n \ll N$. The introduction of a coherent state is not required to account for coherence and interference effects in systems such as Bose-Einstein condensates. 


\subsection{Normal Ordering}

Many expressions (such as the quantum correlation functions or the Hamiltonians) involving annihilation and creation operators are written with these operators already placed in normal order, with creation operators on the left of the annihilation operators. However, not all expressions are normally ordered and it is useful to define the so-called normally ordered forms of other expressions involving the annihilation, creation operators [45]. This applies for both mode and field operators. As operator forms of most interest can be written as linear combinations of products of operators with c-number coefficients, the normally ordered forms of products will be defined first. Thus for two operators

$$
\mathcal{N}\left(\hat{c}_{i} \hat{c}_{j}\right)=\hat{c}_{i} \hat{c}_{j}, \quad \mathcal{N}\left(\hat{c}_{i}^{\dagger} \hat{c}_{j}^{\dagger}\right)=\hat{c}_{i}^{\dagger} \hat{c}_{j}^{\dagger}, \quad \mathcal{N}\left(\hat{c}_{i}^{\dagger} \hat{c}_{j}\right)=\hat{c}_{i}^{\dagger} \hat{c}_{j}, \quad \mathcal{N}\left(\hat{c}_{i} \hat{c}_{j}^{\dagger}\right)=-\hat{c}_{j}^{\dagger} \hat{c}_{i}
$$

The normally ordered form of an operator $\hat{A}$ is typically denoted : $\hat{A}:$ or $\mathcal{N}(\hat{A})$. Note the last result for fermions - the minus sign is becase a permutation of the operators is needed to place the creation operator on the left. In all cases the expressions have all creation operators to the left of all annihilation operators. This property survives for products involving more that two operators, with the creation and annihilation operators separately in their original order. In the fermion case the expression is multiplied by +1 or -1 depending on whether an odd or even permutation of the operators is needed to obtain the normally ordered form. The normally ordered form of a linear combination of products $\hat{P}_{i}$ is defined as the linear combination of the normally ordered products. Thus

$$
\mathcal{N}\left(\gamma_{1} \hat{P}_{1}+\gamma_{2} \hat{P}_{2}\right)=\gamma_{1} \mathcal{N}\left(\hat{P}_{1}\right)+\gamma_{2} \mathcal{N}\left(\hat{P}_{2}\right)
$$

where the $\gamma_{i}$ are c-numbers.

Normally ordered forms have useful features. The vacuum expectation value of any normally ordered form vanishes

$$
\langle 0|\mathcal{N}(\hat{F})| 0\rangle=0
$$

where $\hat{F}$ is a function of the annihilation, creation operators. In the case where the annihilation, creation operators for different modes are involved the following useful theorem can be established

$$
\mathcal{N}\left(F\left(\hat{c}_{i}^{\dagger} \hat{c}_{i}\right) G\left(\hat{c}_{j}^{\dagger} \hat{c}_{j}\right)\right)=\mathcal{N}\left(F\left(\hat{c}_{i}^{\dagger} \hat{c}_{i}\right)\right) \mathcal{N}\left(G\left(\hat{c}_{j}^{\dagger} \hat{c}_{j}\right)\right) \quad(i \neq j)
$$

where $F, G$ are arbitrary functions. This result depends only on the annihilation and creation operators anticommuting for $i \neq j$.

The vacuum state $|0\rangle$, which contains no particles in any mode, is associated with the vacuum projector $|0\rangle\langle 0|$. This projector can be written using normally ordered forms of products of exponential operators based on number operators. We have

$$
\begin{aligned}
|0\rangle\langle 0| & =\mathcal{N}\left(\prod_{i} \exp \left(-\hat{c}_{i}^{\dagger} \hat{c}_{i}\right)\right) \\
& =\mathcal{N}\left[\exp \left(-\sum_{i} \hat{c}_{i}^{\dagger} \hat{c}_{i}\right)\right]
\end{aligned}
$$

in terms of mode operators .

\section{Phase Space Theory}

\subsection{Distribution Function}

In the present phase space theory, each fermion mode is associated with a pair of Grassmann numbers $g_{i}, g_{i}^{+}$- which act as phase space variables. The density operator $\hat{\rho}$.is represented by 
a distribution function $P\left(g, g^{+}\right)$, which is determined from the density operator. The phase space variable $g_{i}$ is associated with the fermion mode annihilation operator $\hat{c}_{i}$ and the phase space variable $g_{i}^{+}$is associated with the fermion mode creation operator $\hat{c}_{i}^{\dagger}$, as will be seen when quantum correlation functions are expressed as phase space integrals involving the distribution function. Note however that Cahill et al [22] employed a phase space which represented the mode creation operators $\hat{c}_{i}^{\dagger}$ by the complex conjugate $g_{i}^{*}$ rather than an unrelated Grassmann variable $g_{i}^{+}$, so their distribution function $P\left(g, g^{*}\right)$ was analogous to the standard $P$ distribution. A similar phase space distribution $B\left(g, g^{*}\right)$ was also used by Plimak et al [23].

It is convenient to introduce the distribution function via the canonical form of the density operator written in terms of Bargmann state projectors, rather than first defining the characteristic function though that approach can also be used. The canonical form of density operator is given in terms of fermion Bargmann states (see Appendix 11).

$$
\hat{\rho}=\int \prod_{i} d g_{i}^{+} \prod_{i} d g_{i} P_{\text {canon }}\left(g, g^{+}\right) \Lambda\left(g, g^{+}\right)
$$

The fermion Bargmann states are defined in Eq. (174) and their relation to the Glauber coherent states is given in Eq. (175). As Grassmann differentials anti-commute we need to specify the convention for their products in (21). This is $\prod_{i} d g_{i}^{+}=d g_{1}^{+} d g_{2}^{+} \cdots d g_{n}^{+}$and $\prod_{i} d g_{i}=d g_{n} \cdots d g_{2} d g_{1}$ - note the reverse order. The quantity $\Lambda\left(g \cdot g^{+}\right)$is the normalised fermion Bargmann state projector

$$
\Lambda\left(g, g^{+}\right)=\frac{|g\rangle_{B B}\left\langle g^{+*}\right|}{\operatorname{Tr}\left(|g\rangle_{B B}\left\langle g^{+*}\right|\right)}=e^{-\mathbf{g} \cdot \mathbf{g}^{+}}|g\rangle_{B B}\left\langle g^{+*}\right|
$$

and the canonical distribution function is given by

$$
P_{\text {canon }}\left(g, g^{+}\right)=\int \prod_{i} d g_{i}^{+*} \prod_{i} d g_{i}^{*}\left\langle\left. g\right|_{B} \hat{\rho} \mid g^{+*}\right\rangle_{B} \exp \left(g \cdot g^{*}+g^{+*} \cdot g^{+}+g \cdot g^{+}\right)
$$

which is obtained from matrix elements of the density operator between Bargmann states. Note the complex conjugates. Here we have $\prod_{i} d g_{i}^{+*}=d g_{1}^{+*} d g_{2}^{+*} \cdots d g_{n}^{+*}$ and $\prod_{i} d g_{i}^{*}=d g_{n}^{*} \cdots d g_{2}^{*} d g_{1}^{*}$ again the order is reversed. The derivation of the canonical form of the density operator is set out in Appendix 12. We point out that the $|g\rangle_{B}$ only depends on the $g_{i}$, and the bra vector $\left\langle g^{+*}\right|$ only depends on the $g_{i}^{+}$. Both the distribution function $P_{\text {canon }}\left(g, g^{+}\right)$and the fermionic Bargmann state projector only depend on the $g_{i}$ and the $g_{i}^{+}$. The complex congugates $g_{i}^{*}$ and the $g_{i}^{+*}$ only appear in the phase space integral (23) giving the distribution function - for example the matrix elements $\left\langle\left. g\right|_{B} \hat{\rho} \mid g^{+*}\right\rangle_{B}$ depend on these complex conjugates. For bosonic systems similar equations are involved in introducing the canonical forms of the density operator for the $P+$ distribution function [16]. However in this case although the $P+$ distribution functions (which are not unique) depend on two independent c-number phase space variables $\alpha_{i}$ and $\alpha_{i}^{+}$for each mode, they also depend on their complex conjugates $\alpha_{i}^{*}$ and $\alpha_{i}^{+*}$. The bosonic Bargmann state projector only depends on the $\alpha_{i}$ and the $\alpha_{i}^{+}$.

The distribution function $P\left(g, g^{+}\right)$is an even Grassmann function of the set of Grassmann variables $\left\{g_{i}\right\},\left\{g_{i}^{+}\right\}$and is of order $2^{n}$. Unlike the positive $P$ function [16] for the boson case, $P\left(g, g^{+}\right)$is unique, as we will demonstrate below via considering the characteristic function. Henceforth we therefore drop the subscript "canon" as it is superfluous. The fermion dstribution function is more analogous to the complex $P$ function for the boson case. For fermion number conserving physical states the distribution function is of the form

$$
P\left(g, g^{+}\right)=P_{0}+\sum_{i, j} P_{2}^{i ; j} g_{i} g_{j}^{+}+\cdots \cdots+P_{2 n}^{12 . . n ; n .21} g_{1} g_{2} . . g_{n} g_{n}^{+} \cdots g_{2}^{+} g_{1}^{+}
$$


where each term contains equal numbers of $g_{i}$ and $g_{i}^{+}$. A total of $2 n ! /(n ! n !)$ c-number coefficients are needed to define the distribution function $P\left(g, g^{+}\right)$.

\subsection{Un-Normalised Distribution Function}

As in Plimak et al [23], it is important for the present work to define the un-normalised $B\left(g, g^{+}\right)$ distribution function, which is related to normalised distribution function $P\left(g, g^{+}\right)$via

$$
B\left(g, g^{+}\right)=P\left(g, g^{+}\right) \exp \left(-\mathbf{g} \cdot \mathbf{g}^{+}\right)
$$

so now the density operator is given by

$$
\hat{\rho}=\int \prod_{i} d g_{i}^{+} \prod_{i} d g_{i} B\left(g, g^{+}\right)\left(|g\rangle_{B}\left\langle\left. g^{+*}\right|_{B}\right)\right.
$$

The use of the un-normalised $B\left(g, g^{+}\right)$function is key to carrying out numerical work with Grassmann phase space methods, as pointed out in [23]. Note again that Plimak et al [23] represented the mode creation operators $\hat{c}_{i}^{\dagger}$ by the complex conjugate $g_{i}^{*}$ rather than an independent Grassmann variable $g_{i}^{+}$.

The normalisation integrals for the $P\left(g, g^{+}\right)$and $B\left(g, g^{+}\right)$distribution function are given in Appendix 13 as Eqs. (206) and (207).

\subsection{Quantum Correlation Functions}

An important aim in quantum-atom optics theory is in determining the quantum correlation functions, which are expectation values of normally ordered products of creation and annihilation operators. These are defined in terms of the density operator $\hat{\rho}$ by

$$
\begin{aligned}
G\left(l_{1}, \cdots, l_{p} ; m_{q}, \cdots, m_{1}\right) & =\left\langle\hat{c}_{l_{1}}^{\dagger} \cdots \hat{c}_{l_{P}}^{\dagger} \hat{c}_{m_{q}} \cdots \hat{c}_{m_{1}}\right\rangle \\
& =\operatorname{Tr}\left\{\left(\hat{c}_{m_{q}}\right) . .\left(\hat{c}_{m_{2}}\right)\left(\hat{c}_{m_{1}}\right) \hat{\rho}\left(\hat{c}_{l_{1}}^{\dagger}\right)\left(\hat{c}_{l_{2}}^{\dagger}\right) . .\left(\hat{c}_{l_{p}}^{\dagger}\right)\right\}
\end{aligned}
$$

for fermions. The quantities $l_{1}, l_{2}, \cdots, l_{p} ; m_{q}, \cdots, m_{2}, m_{1}$. are quantum modes as usual, but now in any order. Normally ordered means that creation operators appear on the left of annihilation operators when the density operator is placed on the left (or right) of all these operators via applying the cyclic property of the trace. The form for the quantum correlation functions - with the density operator placed in the middle - occur in theories of multi particle detection and links the quantum correlation functions to experiment. For physical states of simple fermionic systems - where fermions are not created or destroyed - the quantum correlation functions are zero unless $p=q$.

In phase space theory quantum correlation functions are given by phase space integrals

$$
\begin{aligned}
& \left\langle\left(\hat{c}_{l_{1}}^{\dagger}\right)\left(\hat{c}_{l_{2}}^{\dagger}\right) . .\left(\hat{c}_{l_{p}}^{\dagger}\right)\left(\hat{c}_{m_{q}}\right) . .\left(\hat{c}_{m_{2}}\right)\left(\hat{c}_{m_{1}}\right)\right\rangle \\
= & \int \prod_{i} d g_{i}^{+} \prod_{i} d g_{i} \times\left(g_{m_{q}}\right) . .\left(g_{m_{2}}\right)\left(g_{m_{1}}\right) P\left(g, g^{+}\right)\left(g_{l_{1}}^{+}\right)\left(g_{l_{2}}^{+}\right) . .\left(g_{l_{p}}^{+}\right) \\
= & \int \prod_{i} d g_{i}^{+} \prod_{i} d g_{i} \times\left(g_{m_{q}}\right) . .\left(g_{m_{2}}\right)\left(g_{m_{1}}\right) B\left(g, g^{+}\right) \exp \left(\mathbf{g} \cdot \mathbf{g}^{+}\right)\left(g_{l_{1}}^{+}\right)\left(g_{l_{2}}^{+}\right) . .\left(g_{l_{p}}^{+}\right)
\end{aligned}
$$

The derivation of this important result is set out in Appendix 13. Note that can replace the exponential via $\prod_{i \neq\{l\},\{m\}}\left(1+g_{i} g_{i}^{+}\right)$for the $B$ distribution result. For physical states the phase space integrals give zero unless $p=q$ as required, since for these states the distribution function has the form (24), and the same number of $g_{m_{i}}$ as $g_{l_{j}}^{+}$are needed to give a non-zero integral. 


\subsection{Fock State Populations, Coherences}

Another set of important quantities that need to be determined are the populations of and coherences between the fermion Fock states. We consider two Fock states - each with $p$ occupied modes - given by

$$
|\Phi\{l\}\rangle=\left(\hat{c}_{l_{1}}^{\dagger}\right)\left(\hat{c}_{l_{2}}^{\dagger}\right) . .\left(\hat{c}_{l_{p}}^{\dagger}\right)|0\rangle \quad|\Phi\{m\}\rangle=\left(\hat{c}_{m_{1}}^{\dagger}\right)\left(\hat{c}_{m_{2}}^{\dagger}\right) . .\left(\hat{c}_{m_{p}}^{\dagger}\right)|0\rangle
$$

The population of the Fock state $|\Phi\{l\}\rangle$ is given by the expectation value of the projector $|\Phi\{l\}\rangle\langle\Phi\{l\}|$ onto this state

$$
\begin{aligned}
P(\Phi\{l\}) & =\operatorname{Tr}(|\Phi\{l\}\rangle\langle\Phi\{l\}| \hat{\rho}) \\
& =\int \prod_{i} d g_{i}^{+} \prod_{i} d g_{i}\left(g_{l_{p}}\right) . .\left(g_{l_{2}}\right)\left(g_{l_{1}}\right) B\left(g, g^{+}\right)\left(g_{l_{1}}^{+}\right)\left(g_{l_{2}}^{+}\right) . .\left(g_{l_{p}}^{+}\right)
\end{aligned}
$$

and the coherence between the Fock states $|\Phi\{l\}\rangle$ and $|\Phi\{m\}\rangle$ is given by the expectation value of the transition operator $|\Phi\{m\}\rangle\langle\Phi\{l\}|$ between the two states

$$
\begin{aligned}
C(\Phi\{m\}, \Phi\{l\}) & =\operatorname{Tr}(|\Phi\{m\}\rangle\langle\Phi\{l\}| \hat{\rho}) \\
& =\int \prod_{i} d g_{i}^{+} \prod_{i} d g_{i}\left(g_{l_{p}}\right) . .\left(g_{l_{2}}\right)\left(g_{l_{1}}\right) B\left(g, g^{+}\right)\left(g_{m_{1}}^{+}\right)\left(g_{m_{2}}^{+}\right) . .\left(g_{m_{p}}^{+}\right)
\end{aligned}
$$

In both cases the results are obtained as Grassmann phase space integrals, which are in simple form when un-normalised $B$ distribution function is used to represent the density operator. The proof of these important results is similar to that given in Appendix 13 for the quantum correlation functions, but now we use Eq.(26) for the density operator rather than (21) and the evaluation of the trace is more complicated. The derivation is given in Appendix 13.

\subsection{Characteristic Function}

For completeness, we also define the characteristic function

$$
\begin{aligned}
\chi\left(h, h^{+}\right) & =\operatorname{Tr}\left(\hat{\Omega}^{+}\left(h^{+}\right) \hat{\rho} \hat{\Omega}^{-}(h)\right) \\
\hat{\Omega}^{+}\left(h^{+}\right) & =\exp i \sum_{i} \hat{c}_{i} h_{i}^{+}=\prod_{i}\left(1+i \hat{c}_{i} h_{i}^{+}\right) \\
\hat{\Omega}^{-}(h) & =\exp i \sum_{i} h_{i} \hat{c}_{i}^{\dagger}=\prod_{i}\left(1+i h_{i} \hat{c}_{i}^{\dagger}\right)
\end{aligned}
$$

The characteristic function is also defined in terms of the density operator and is a function of Grassmann variables $\left\{h_{i}, h_{i}^{+}\right\}$. Its definition involves Grassmann operators $\hat{\Omega}^{+}\left(h^{+}\right)$and $\hat{\Omega}^{-}(h)$, which are exponential forms involving the fermion mode annihilation and creation operators. For fermion number conserving physical states the characteristic function has a form analogous to $(24)$.

The distribution function $P\left(g, g^{+}\right)$in Eq. (23) is related to the characteristic function via.

$$
\begin{aligned}
& \chi\left(h, h^{+}\right) \\
= & \int \prod_{i} d g_{i}^{+} \prod_{i} d g_{i} \exp i \sum_{i}\left\{g_{i} h_{i}^{+}\right\} P\left(g, g^{+}\right) \exp i \sum_{i}\left\{h_{i} g_{i}^{+}\right\} \\
= & \int \prod_{i} d g_{i}^{+} \prod_{i} d g_{i} \prod_{i}\left(1+i\left\{g_{i} h_{i}^{+}\right\}\right) P\left(g, g^{+}\right) \prod_{i}\left(1+i\left\{h_{i} g_{i}^{+}\right\}\right)
\end{aligned}
$$


where the convention is $\prod_{i} d g_{i}^{+} \prod_{i} d g_{i}=d g_{1}^{+} d g_{2}^{+} \cdots d g_{n}^{+} d g_{n} c d o t s d g_{2} d g_{1}$, as before. This result is easily obtained by substituting the canonical form of the density operator into the definition of the characteristic function, and then evaluating the effect of the operators $\hat{\Omega}_{f}^{+}\left(h^{+}\right)$and $\hat{\Omega}_{f}^{-}(h)$ on the Bargmann states, following a similar proceedure as in Appendix 13.

From its definition, it is apparent that the characteristic function is uniquely determined by the density operator - hence its name. In an alternative approach to introducing the distribution function, the requirement that it is linked to the chararacteristic function as in (34) is taken its basic definition. However, having established that the canonical distribution function is related to the characteristic function as in (34), this result implies that the fermion distribution function is in fact unique. Using (34) with a different distribution function $P_{f}\left(\mathbf{g}, \mathbf{g}^{+}\right)$it is easy to show that

$$
0=\int d \mathbf{g}^{+} d \mathbf{g} \exp \left(i \mathbf{g} \cdot \mathbf{h}^{+}\right)\left[P_{f}\left(\mathbf{g}, \mathbf{g}^{+}\right)-P_{\text {canon }}\left(\mathbf{g}, \mathbf{g}^{+}\right)\right] \exp \left(i \mathbf{h} \cdot \mathbf{g}^{+}\right)
$$

for all $\mathbf{h}, \mathbf{h}^{+}$. It then follows - for example by writing $P_{f}\left(\mathbf{g}, \mathbf{g}^{+}\right)-P_{\text {canon }}\left(\mathbf{g}, \mathbf{g}^{+}\right)$in the form $(24)$ - that

$$
P_{f}\left(\mathbf{g}, \mathbf{g}^{+}\right)=P_{\text {canon }}\left(\mathbf{g}, \mathbf{g}^{+}\right)
$$

which establishes the uniqueness of the fermion distribution function.

Having established the link in (34) between the characteristic function and the distribution function, the expression for quantum correlation functions as phase space integrals can be derived in a second way. We first apply Grassmann differentiation to the characteristic function (32) the appropriate number of times $(p)$ from the left with respect to the $h_{i}^{+}$and from the right (q) with respect to the $h_{j}$, and then let the $h_{i}, h_{i}^{+}$all become zero. The differentiation of the expression under the trace operation requires $p-q$ to be an even number. Applying the same differentiation processes to the characteristic function in (34) and letting the $h_{i}, h_{i}^{+}$all become zero, then establishes the phase space integral result (28) involving the $P$ distribution.

\section{Fokker-Planck Equation}

Here we obtain the Fokker-Planck equations for the distribution function that are equivalent to the Liouville von-Neumann, master or Matsubara equations [46] for the density operator for fermions. This is accomplished via the application of a standard set of rules known as the Correspondence Rules, which relate the effect of replacing the density operator by its product with annihilation or creation operators. The Fokker-Planck equations are established for both the un-normalised and normalised distribution functions. If the Fokker-Planck equation contains only first and second order derivatives of the phase variables, we show in Section 5 that the Fokker-Planck equation can be replaced by Ito stochastic equations for stochastic variables that replace the phase space Grassmann variables. Stochastic averages of products of the stochastic phase space variables then can be used to determine the quantum correlation functions, Fock state populations and coherences instead of using phase space averages based on the distribution function. From the point of view of efficient numerical calculations of the quantum correlation functions or Fock state populations and coherences, the use of stochastic methods is preferable, as they in effect avoid having to determine the form of the distribution function over the whole of Grassmann phase space. 


\subsection{Hamiltonian}

The Hamiltonian for a fermion system involving one and two particle interactions may be written in terms of one particle and two particle operators $\hat{h}(a)$ and $\hat{V}(a, b)=\hat{V}(b, a)$ as

$$
\hat{H}=\sum_{A} \hat{h}(a)+\frac{1}{2} \sum_{a, b} \hat{V}(a, b)
$$

where the sums are over the $N$ identical particles and where the expressions are invariant under any permutation of the identical particles. In terms of annihilation and creation operators the Hamiltonian given in first quantisation in the form (37) can be written in the second quantisation form as

$$
\hat{H}_{f}=\sum_{i, j} h_{i j} \hat{c}_{i}^{\dagger} \hat{c}_{j}+\frac{1}{2} \sum_{i, j, k, l} v_{i j k l} \hat{c}_{i}^{\dagger} \hat{c}_{j}^{\dagger} \hat{c}_{l} \hat{c}_{k}
$$

where the coefficients are given in terms of single particle states (modes) via one or two particle matrix elements as

$$
h_{i j}=\left\langle\phi_{i}(a)|\hat{h}(a)| \phi_{j}(a)\right\rangle \quad v_{i j k l}=\left\langle\phi_{i}(a)\left|\left\langle\phi_{j}(b)|\hat{V}(a, b)| \phi_{k}(a)\right\rangle\right| \phi_{l}(b)\right\rangle
$$

\subsection{Master Equation}

The evolution of the quantum state allowing for both Hamiltonian dynamics and Markovian coupling to an external reservoir is determined from the master equation

$$
\frac{\partial}{\partial t} \hat{\rho}=\frac{1}{i \hbar}[\hat{H}, \hat{\rho}]+\frac{1}{2} \sum_{a, b} \Gamma_{a b}\left(2 \hat{S}_{b}^{\dagger} \hat{\rho} \hat{S}_{a}-\hat{\rho} \hat{S}_{a} \hat{S}_{b}^{\dagger}-\hat{S}_{a} \hat{S}_{b}^{\dagger} \hat{\rho}\right)
$$

where in the relaxation terms the fermion system transition operators $\hat{S}_{a}, \hat{S}_{b}$ are associated with pairs fermion modes and are denoted $a \equiv i, j$ and $b \equiv k, l$

$$
\begin{aligned}
\hat{S}_{a} & =\hat{c}_{j}^{\dagger} \hat{c}_{i} \quad \hat{S}_{b}=\hat{c}_{l}^{\dagger} \hat{c}_{k} \\
\Gamma_{a b} & \equiv \Gamma_{i j ; k l}
\end{aligned}
$$

From the matrix elements in (39) and from the hermitiancy requirement for the evolved density operator, certain symmetries

$$
h_{i j}=h_{j i}^{*} \quad v_{i j k l}=v_{j i l k}=v_{k l j i}^{*} \quad \Gamma_{i j ; k l}=\Gamma_{k l ; i j}^{*}
$$

apply to the Hamiltonian and relaxation quantities occuring in the mater equation. The Liouvillevon Neumann equation for Hamiltonian dynamics is obtained by ignoring the relaxation terms. The Matsubara equation [46] allows for temperature evolution of the density operator that describes thermal equilibrium states, but will not be treated here.

\subsection{Correspondence Rules - B Distribution Function}

The correspondence rules are derived from the canonical forms for density operator and Grassmann differentiation results for Bargmann states

$$
\begin{aligned}
& \hat{c}_{i}^{\dagger}|g\rangle_{B}=\left(-\frac{\vec{\partial}}{\partial g_{i}}\right)|g\rangle_{B} \quad\left\langle\left. g^{+*}\right|_{B} \hat{c}_{i}=\left\langle\left. g^{+*}\right|_{B}\left(-\frac{\overleftarrow{\partial}}{\partial g_{i}^{+}}\right)\right.\right. \\
& \hat{c}_{i}|g\rangle_{B}=g_{i}|g\rangle_{B} . \quad\left\langle\left. g^{+*}\right|_{B} \hat{c}_{i}^{\dagger}=\left\langle\left. g\right|_{B} g_{i}^{+}\right.\right.
\end{aligned}
$$


together with the eigenvalue equations for Bargmann states from (179). The differentiation rules are easily derived from the Bargmann state definition (174).

The correspondence rules applying the the fermionic $B$ distribution function are:

$$
\begin{aligned}
& \hat{\rho} \Rightarrow \hat{c}_{i} \hat{\rho} \quad B\left(g, g^{+}\right) \Rightarrow g_{i} B\left(g, g^{+}\right) \\
& \hat{\rho} \Rightarrow \hat{\rho} \hat{c}_{i} \quad B\left(g, g^{+}\right) \Rightarrow B\left(g, g^{+}\right)\left(+\frac{\overleftarrow{\partial}}{\partial g_{i}^{+}}\right) \\
& \hat{\rho} \Rightarrow \hat{c}_{i}^{\dagger} \hat{\rho} \quad B\left(g, g^{+}\right) \Rightarrow\left(+\frac{\vec{\partial}}{\partial g_{i}}\right) B\left(g, g^{+}\right) \\
& \hat{\rho} \Rightarrow \hat{\rho} \hat{c}_{i}^{\dagger} \quad B\left(g, g^{+}\right) \Rightarrow B\left(g, g^{+}\right) g_{i}^{+}
\end{aligned}
$$

Thus if the density operator $\hat{\rho}$ is replaced by $\hat{c}_{i} \hat{\rho}$, then the $B$ distribution function is replaced by $g_{i} B\left(g, g^{+}\right)$, whereas if the density operator $\hat{\rho}$ is replaced by $\hat{\rho} \hat{c}_{i}$, then the $B$ distribution function is replaced by $B\left(g, g^{+}\right)\left(+\frac{\overleftarrow{\partial}}{\partial g_{i}^{+}}\right)$. The derivation of the correspondence rules is set out in Appendix 14 and involves using the canonical form (26) for the density operator in conjunction with Grassmann integration by parts rules and the evenness feature of the quantities involved. The first step involves applying (44) to the Bargmann state projectors $|g\rangle_{B}\left\langle\left. g^{+*}\right|_{B}\right.$ so only derivatives with respect to $g_{i}, g_{i}^{+}$occur. Alternative correspondence rules such as

$$
\hat{\rho} \Rightarrow \hat{\rho} \hat{c}_{i} \quad B\left(g, g^{+}\right) \Rightarrow\left(-\frac{\vec{\partial}}{\partial g_{i}^{+}}\right) B\left(g, g^{+}\right)
$$

can be obtained using (144), but these alternatives can lead to incorrect results when the correspondence rules are applied in succession, so will not be used here. The correspondence rules can also be derived via the characteristic function, but that proceedure is more complicated.

The correspondence rules can be applied in succession, for example

$$
\begin{aligned}
\hat{\rho} & \Rightarrow \hat{c}_{i}^{\dagger} \hat{\rho} \hat{c}_{j} \\
B\left(g, g^{+}\right) & \Rightarrow\left(\left(+\frac{\vec{\partial}}{\partial g_{i}}\right) B\left(g, g^{+}\right)\right)\left(+\frac{\overleftarrow{\partial}}{\partial g_{j}^{+}}\right) \\
& =\left(+\frac{\vec{\partial}}{\partial g_{i}}\right)\left(B\left(g, g^{+}\right)\left(+\frac{\overleftarrow{\partial}}{\partial g_{j}^{+}}\right)\right)
\end{aligned}
$$

The two equal results - which are based on the mixed derivative formula (152) - show that the replacements can be carried out in either order.

Note that combinations of Grassmann variables and derivatives absent in the correspondence rules for the $B$ distribution function, such combinations being present in the rules for the $P$ distribution. As Plimak et al [23] pointed out, the absence of such combinations is vital for the drift term in the Fokker-Planck equation to depend linearly on the Grassmann variables, a key feature enabling numerically solvable forms for the related Ito stochastic equations to be obtained. For completeness, the correspondence rules for the $P$ distribution function are listed in Appendix 14.

Plimak et al [23] treated a distribution function $B\left(g, g^{*}\right)$ and Bargman state projectors 
$|g\rangle_{B}\left\langle\left. g^{*}\right|_{B}\right.$ that depended on $g, g^{*}$ so their correspondence rules are

$$
\begin{array}{ll}
\hat{\rho} \Rightarrow \hat{c}_{i} \hat{\rho} & B\left(g, g^{*}\right) \Rightarrow g_{i} B\left(g, g^{*}\right) \\
\hat{\rho} \Rightarrow \hat{\rho} \hat{c}_{i} & B\left(g, g^{*}\right) \Rightarrow B\left(g, g^{*}\right)\left(+\frac{\overleftarrow{\partial}}{\partial g_{i}^{*}}\right) \\
\hat{\rho} \Rightarrow \hat{c}_{i}^{\dagger} \hat{\rho} & B\left(g, g^{*}\right) \Rightarrow\left(+\frac{\vec{\partial}}{\partial g_{i}}\right) B\left(g, g^{*}\right) \\
\hat{\rho} \Rightarrow \hat{\rho} \hat{c}_{i}^{\dagger} & B\left(g, g^{*}\right) \Rightarrow B\left(g, g^{*}\right) g_{i}^{*}
\end{array}
$$

\subsection{Fokker-Planck Equation - B Distribution Function}

By applying the correspondence rules (45) the master equation for the density operator is equivalent to the Fokker-Planck equation for the $B$ distribution function

$$
\frac{\partial}{\partial t} B\left(g, g^{+}\right)=-\sum_{p=1}^{2 n}\left(A_{p} B\left(g, g^{+}\right)\right) \frac{\overleftarrow{\partial}}{\partial g_{p}}+\frac{1}{2} \sum_{p, q=1}^{2 n}\left(D_{p q} B\left(g, g^{+}\right)\right) \frac{\overleftarrow{\partial}}{\partial g_{q}} \frac{\overleftarrow{\partial}}{\partial g_{p}}
$$

Here we have listed the $g_{i}, g_{i}^{+}$as $\left\{g_{p}\right\} \equiv\left\{g_{1}, g_{2}, \cdots, g_{n}, g_{1}^{+}, g_{2}^{+}, \cdots, g_{n}^{+}\right\}$. Note that only derivatives with respect to $g_{i}, g_{i}^{+}$occur (and not the complex conjugates $g_{i}^{*}, g_{i}^{+*}$ ). The FokkerPlanck equation can be expressed in several ways using (144), (151), (152) and (153), but here we have chosen the form that only involves right derivatives, as this facilitates the derivation of the Ito stochastic equations. The term involving first order derivatives is the drift term and that involving second order derivatives is the diffusion term.

We note that for a distribution function $B\left(g, g^{*}\right)$ (as considered by Plimak et al [23]) the Fokker-Planck equations for time evolution would involve derivatives with respect to $g_{i}, g_{i}^{*}$. However, as Plimak et al [23] only considered Matsubara equations for thermal evolution rather than a master equation, only terms of the form $\hat{H} \hat{\rho}$ (and not $\hat{\rho} \hat{H}$ ) are involved, so that only the first and third correspondence rule in (48) would be needed. Hence their Fokker-Planck equation would only involve derivatives with respect to $g_{i}$ and not the complex conjugates $g_{i}^{*}$. Similarly, the drift, diffusion matrices would also not involve $g_{i}^{*}$.

The drift vector $A$ and the diffusion matrix $D$ can be written in terms of sub-matrices as ( $T$ is the transpose).

$$
[A]=\left[\begin{array}{l}
C^{-} \\
C^{+}
\end{array}\right] \quad[D]=\left[\begin{array}{cc}
-F^{--} & +F^{-+} \\
-\left(F^{-+}\right)^{T} & +F^{++}
\end{array}\right]
$$

where the coefficients giving drift vector and diffusion matrix elements are

$$
\begin{gathered}
C_{i}^{-}=-\frac{1}{i \hbar} \sum_{j} h_{i j} g_{j}+\sum_{j k}\left(\frac{1}{2} \Gamma_{k i k j}\right) g_{j} \\
C_{i}^{+}=+\frac{1}{i \hbar} \sum_{j} h_{i j}^{*} g_{j}^{+}+\sum_{j k} g_{j}^{+}\left(\frac{1}{2} \Gamma_{k i k j}^{*}\right) \\
F_{i j}^{--}=\frac{1}{i \hbar} \sum_{k l} \nu_{i j k l} g_{l} g_{k}+\sum_{k l} \frac{1}{2}\left\{\Gamma_{l i j k}+\Gamma_{k j i l}\right\} g_{l} g_{k}=-F_{j i}^{--} \\
F_{i j}^{++}=-\frac{1}{i \hbar} \sum_{k l} \nu_{i j k l}^{*} g_{k}^{+} g_{l}^{+}+\sum_{k l} \frac{1}{2}\left\{\Gamma_{l i j k}^{*}+\Gamma_{k j i l}^{*}\right\} g_{k}^{+} g_{l}^{+}=-F_{j i}^{++} \\
F_{i j}^{-+}=\sum_{k l}\left\{\Gamma_{j k i l}\right\} g_{l} g_{k}^{+} \quad F_{i j}^{+-}=\sum_{k l}\left\{\Gamma_{j k i l}^{*}\right\} g_{l}^{+} g_{k}=-F_{j i}^{-+}
\end{gathered}
$$


In these submatrices $i, j=1,2, \cdots, n$ and the c-number quantities $h_{i j}, \Gamma_{k i k j}, \nu_{i j k l}$ etc are defined in (39) and (42).

We see that the drift vector is an odd Grassmann function, and depends linearly dependent on Grassmann variables. The diffusion matrix is an even Grassmann function and using (43) we can show that it is anti-symmetric $D_{p q}=-D_{q p}$. The diffusion matrix elements depend quadratically on the Grassmann variables.

For completeness, the Fokker-Planck equation and the drift vector, diffusion matrix elements for the $P$ distribution function are set out in Appendix 15. We note that here the drift vector includes terms that depend cubically on the Grassmann variables. Although the $P$ distribution function is still equivalent to Ito stochastic equations, the third order feature of the drift vector results in the Ito equations not being of use in numerical calculations. However, the $P$ distribution function and its equivalent Ito stochastic equations are still useful for formal theory, hence they are recorded in the present paper.

\section{Ito Stochastic Equations}

\subsection{Key Idea}

The basis idea for establishing Ito stochastic equations that are equivalent to the Fokker-Planck equations is to consider an arbitrary function $F\left(g, g^{+}\right)$of the phase space variables $g_{p}$ and its phase space average $\left\langle F\left(g, g^{+}\right)\right\rangle_{t}$ as defined by a phase space integral of the product of $F\left(g, g^{+}\right)$ with the distribution function $B\left(g, g^{+}, t\right)$. The time evolution of the phase space average $\left\langle F\left(g, g^{+}\right)\right\rangle$is then determined from the time dependence of the distribution function, which is governed by the Fokker-Planck equation. The time dependent behaviour of $\left\langle F\left(g, g^{+}\right)\right\rangle$could be obtained by solving the Fokker-Planck equation for all phase space variables $g, g^{+}$and then computing the phase space integral of the product of $F\left(g, g^{+}\right)$with $B\left(g, g^{+}, t\right)$. This is obviously difficult to carry out numerically, especially as Grassmann functions are involved. Instead, the phase space variables $g_{p}$ are replaced by time dependent stochastic variables $\tilde{g}_{p}(t)$, where $\tilde{g}_{p k}(t)$ is $k$ th member of a stochastic ensemble $k=1,2, \cdots, m$. The stochastic variables $\tilde{g}_{p}(t)$ will determined as solutions of a suitably chosen Ito stochastic equation. The stochastic average of $F\left(g, g^{+}\right)$at time $t$ is defined by substituting $g, g^{+}$with the stochastic Grassmann variables $\tilde{g}_{i}(t), \widetilde{g}_{i}{ }^{+}(t)$. to give $F\left(\tilde{g}(t), \tilde{g}^{+}(t)\right)$ and then averaging over members of the ensemble to give the stochastic average $\overline{F\left(\tilde{g}(t), \tilde{g}^{+}(t)\right)}$. The overall idea is that this ensemble of time dependent stochastic variables $\tilde{g}_{p}(t)$ provides a representative sampling of the distribution function such that the stochastic average $\overline{F\left(\tilde{g}(t), \tilde{g}^{+}(t)\right)}$.for any arbitrary function $F\left(g, g^{+}\right)$gives the same result as would be obtained from the phase space integral involving the distribution function.

Thus, the phase space average at any time $t$ of the arbitary function $F\left(g, g^{+}\right)$and stochastic average of same function are required to coincide, and for this to occur the Ito stochastic equations for $\widetilde{g}_{p}(t)$ must be suitably related to Fokker-Planck equation for distribution function $B\left(g, g^{+}, t\right)$.

$$
\left\langle F\left(g, g^{+}\right)\right\rangle_{t}=\overline{F\left(\tilde{g}(t), \tilde{g}^{+}(t)\right)}
$$

This approach is based on a treatment by Gardiner (see pp 95-96 in [47]) which established the relationship between Fokker-Planck and Ito stochastic equations for bosons. It needs to be emphasised that both sides of (53) are c-numbers - the left side involves Grassmann phase space integrals of the product of a Grassmann function $F$ and the Grassmann distribution function (and the result depends just on the c-numbers specifying these Grassmann functions of the phase space variables), whereas the right side involves a stochastic average over the same function 
$F$, but now of stochastic Grassmann variables determined from Ito stochastic equations (and the result depends just on the stochastic averages of products of c-number Gaussian-Markov random noise terms, on stochastic averages of products of the initial stochastic Grassmann phases space variables obtained from the initial conditions and on the c-numbers specifying $F$ ). That the right side is a c-number is further seen in Section 5.9.

\subsection{Phase Space Averages- B Distribution Function}

As indicated before, the phase space average of $F\left(g, g^{+}\right)$is defined by the phase space integal

$$
\left\langle F\left(g, g^{+}\right)\right\rangle_{t}=\iint d g^{+} d g F\left(g, g^{+}\right) B\left(g, g^{+}, t\right)
$$

Typical examples of such expressions are the phase space integrals (28), (30) and (31) giving the quantum correlation functions, Fock state populations and coherences, from which the particular forms for $F\left(g, g^{+}\right)$can be read off.

It is straight-forward to derive an expression for the time derivative of the phase space average, noting that the time dependence is embodied in the $B$ distribution function - which satisfies the Fokker-Planck equation (49). Using integration by parts we find that

$$
\begin{aligned}
\frac{d}{d t}\left\langle F\left(g, g^{+}\right)\right\rangle_{t} & =\left(\frac{d}{d t}\left\langle F\left(g, g^{+}\right)\right\rangle_{t}\right)_{\text {Drift }}+\left(\frac{d}{d t}\left\langle F\left(g, g^{+}\right)\right\rangle_{t}\right)_{\text {Diff } n} \\
\left(\frac{d}{d t}\left\langle F\left(g, g^{+}\right)\right\rangle_{t}\right)_{\text {Drift }} & =\left\langle\left\{-\sum_{p=1}^{2 n}\left[F\left(g, g^{+}\right) \frac{\overleftarrow{\partial}}{\partial g_{p}}\right]\left(A_{p}\right)\right\}\right\rangle_{t} \\
\left(\frac{d}{d t}\left\langle F\left(g, g^{+}\right)\right\rangle_{t}\right)_{D i f f n} & =\left\langle\left\{+\frac{1}{2} \sum_{p, q=1}^{2 n}\left[F\left(g, g^{+}\right) \frac{\overleftarrow{\partial}}{\partial g_{p}} \frac{\overleftarrow{\partial}}{\partial g_{q}}\right]\left(D_{q p}\right)\right\}\right\rangle_{t}
\end{aligned}
$$

The time derivative of the phase space average is the sum of two terms, one associated with the drift term in the Fokker-Planck equation, the other with the diffusion term. This expression for the time derivative of the phase space average will be equated to the time derivative of the stochastic average.

\subsection{Stochastic Averages}

As indicated before the stochastic average of $F\left(g, g^{+}\right)$is defined by

$$
\overline{F\left(\tilde{g}(t), \tilde{g}^{+}(t)\right)}=\frac{1}{m} \sum_{k=1}^{m} f\left(\tilde{g}_{p k}(t)\right)
$$

where $F\left(\tilde{g}_{i k}(t), \tilde{g}_{i k}^{+}(t)\right)=f\left(\tilde{g}_{p k}(t)\right)$, and we have averaged $F\left(\tilde{g}_{i k}(t), \tilde{g}_{i k}^{+}(t)\right)$ at time $t$ over all $m$ members of the ensemble of stochastic variables. Some important properties regarding the stochastic averages of sums and products, together with the concept of uncorrelated stochastic averages are set out in Appendix 16.

We now can write the Fock state populations, coherences as stochastic average of the relevant product of stochastic Grassmann variables

$$
\begin{aligned}
P(\Phi\{l\})_{t} & =\overline{\left(\tilde{g}_{l_{p}}(t) \cdot \tilde{g}_{l_{1}}(t)\right)\left(\tilde{g}_{l_{1}}^{+}(t) \cdots \tilde{g}_{l_{p}}^{+}(t)\right)} \\
C(\Phi\{m\}, \Phi\{l\})_{t} & =\overline{\left(\tilde{g}_{l_{p}}(t) \cdot \tilde{g}_{l_{1}}(t)\right)\left(\tilde{g}_{m_{1}}^{+}(t) \cdots \tilde{g}_{m_{p}}^{+}(t)\right)}
\end{aligned}
$$


Similarly, for the quantum correlation function.

$$
=\frac{\left\langle\left(\hat{c}_{l_{1}}^{\dagger}\right)\left(\hat{c}_{l_{2}}^{\dagger}\right) . .\left(\hat{c}_{l_{p}}^{\dagger}\right)\left(\hat{c}_{m_{q}}\right) . .\left(\hat{c}_{m_{2}}\right)\left(\hat{c}_{m_{1}}\right)\right\rangle}{\left(\tilde{g}_{m_{q}}\right) . .\left(\tilde{g}_{m_{2}}\right)\left(\tilde{g}_{m_{1}}\right) \exp \left(\tilde{g} \cdot \tilde{g}^{+}\right)\left(\tilde{g}_{l_{1}}^{+}\right)\left(\tilde{g}_{l_{2}}^{+}\right) . .\left(\tilde{g}_{l_{p}}^{+}\right)}
$$

Once the Ito stochastic equations for the Grassmann stochastic variables are obtained and solved, we can then replace the phase space integral results by the equivalent stochastic averages to determine the results for the quantum correlation functions, Fock state populations and coherences.

\subsection{Ito Stochastic Eqns for Grassmann Variables}

The Ito stochastic equations are assumed to be of the form

$$
\frac{d}{d t} \tilde{g}_{p}(t)=C^{p}(\tilde{g}(t))+\sum_{a} B_{a}^{p}(\tilde{g}(t)) \Gamma_{a}\left(t_{+}\right)
$$

where $C^{p}(\tilde{g}(t))$ and $B_{a}^{p}(\tilde{g}(t))$ are odd Grassmann functions which are to be determined. The quantities $\Gamma_{a}\left(t_{+}\right)$are c-number Gaussian-Markoff random noise terms [13].

The basic stochastic average properties of the Gaussian-Markoff random noise terms are

$$
\begin{aligned}
\overline{\Gamma_{a}\left(t_{1}\right)}= & 0 \\
\overline{\Gamma_{a}\left(t_{1}\right) \Gamma_{b}\left(t_{2}\right)}= & \delta_{a b} \delta\left(t_{1}-t_{2}\right) \\
\overline{\Gamma_{a}\left(t_{1}\right) \Gamma_{b}\left(t_{2}\right) \Gamma_{c}\left(t_{3}\right)}= & 0 \\
\overline{\Gamma_{a}\left(t_{1}\right) \Gamma_{b}\left(t_{2}\right) \Gamma_{c}\left(t_{3}\right) \Gamma_{d}\left(t_{4}\right)}= & \overline{\Gamma_{a}\left(t_{1}\right) \Gamma_{b}\left(t_{2}\right)} \overline{\Gamma_{c}\left(t_{3}\right) \Gamma_{d}\left(t_{4}\right)}+\overline{\Gamma_{a}\left(t_{1}\right) \Gamma_{c}\left(t_{3}\right)} \overline{\Gamma_{b}\left(t_{2}\right) \Gamma_{d}\left(t_{4}\right)} \\
& +\overline{\Gamma_{a}\left(t_{1}\right) \Gamma_{d}\left(t_{4}\right)} \overline{\Gamma_{b}\left(t_{2}\right) \Gamma_{c}\left(t_{3}\right)}
\end{aligned}
$$

showing that the stochastic averages of a single $\Gamma$ is zero and the stochastic average of the product of two $\Gamma$ 's is zero if they are different and delta function correlated in the time difference if they are the same. In addition, the stochastic averages of products of odd numbers of $\Gamma$ are zero and stochastic averages of products of even numbers of $\Gamma$ are the sums of products of stochastic averages of pairs of $\Gamma$. At this stage we just list the $\Gamma_{a}$ via $a=1,2, \cdots, i(n)$, where the total number $i(n)$ is expected to depend on the number of modes $n$. It will turn out that $i(n)=2 n^{2}$.

A further important property is that any $F(\tilde{g}(t))$ and the products of any $\Gamma_{a}\left(t_{+}\right)$at later times $t_{+}$are uncorrelated

$$
=\frac{\overline{F\left(\tilde{g}\left(t_{1}\right)\right) \Gamma_{a}\left(t_{2}\right) \Gamma_{b}\left(t_{3}\right) \Gamma_{c}\left(t_{4}\right) . . \Gamma_{k}\left(t_{l}\right)}}{F\left(\tilde{g}\left(t_{1}\right)\right)} \overline{\Gamma_{a}\left(t_{2}\right) \Gamma_{b}\left(t_{3}\right) \Gamma_{c}\left(t_{4}\right) . . \Gamma_{k}\left(t_{l}\right)} \quad t_{1}<t_{2}, t_{3}, \cdots, t_{l}
$$

Together with the stochastic averaging properties, the uncorrelation property and stochastic properties of sums and products, an expression for the time derivative of the stochastic average of $F\left(\tilde{g}, \widetilde{g}^{+}\right)$can be derived, which involves the $C^{p}(\tilde{g}(t))$ and $B_{a}^{p}(\tilde{g}(t))$ Grassman functions in the Ito stochastic equations.

We can also write the Ito stochastic equation for $\tilde{g}_{p}(t)$ in the integral form

$$
\begin{aligned}
\tilde{g}_{p}(t+\delta t)-\tilde{g}_{p}(t) & =C^{p}\left(\tilde{g}_{q}(t)\right) \delta t+\sum_{a} B_{a}^{p}\left(\tilde{g}_{q}(t)\right) \int_{t}^{t+\delta t} d t_{1} \Gamma_{a}\left(t_{1}\right) \\
\delta \tilde{g}_{p}(t) & =C^{p}\left(\tilde{g}_{q}(t)\right) \delta t+\sum_{a} B_{a}^{p}\left(\tilde{g}_{q}(t)\right) \delta \tilde{w}_{a}\left(t_{+}\right)
\end{aligned}
$$


where

$$
\delta \tilde{g}_{p}(t)=\tilde{g}_{p}(t+\delta t)-\tilde{g}_{p}(t)
$$

is a Grassmann stochastic increment and the Wiener stochastic variable $\tilde{w}_{a}(t)$ and the Wiener increment are

$$
\tilde{w}_{a}(t)=\int_{t_{0}}^{t} d t_{1} \Gamma_{a}\left(t_{1}\right) \quad \delta \tilde{w}_{a}\left(t_{+}\right)=\int_{t+}^{t+\delta t} d t_{1} \Gamma_{a}\left(t_{1}\right)
$$

An important result for the stochastic average of the product of two Wiener increments is

$$
\overline{\delta \tilde{w}_{a}\left(t_{+}\right) \delta \tilde{w}_{b}\left(t_{+}\right)}=\delta_{a, b} \delta t
$$

which is easily shown using (61).

We find that the time derivative of the stochastic average of $F\left(\tilde{g}, \tilde{g}^{+}\right)$is given by

$$
\frac{\frac{d}{d t} \overline{F\left(\tilde{g}(t), \tilde{g}^{+}(t)\right)}=\overline{\sum_{p}\left[F\left(g, g^{+}\right) \frac{\overleftarrow{\partial}}{\partial g_{p}}\right] C^{p}(\tilde{g}(t))}}{+\frac{1}{2} \sum_{p} \sum_{q}\left[F\left(g, g^{+}\right) \frac{\overleftarrow{\partial}}{\partial g_{p}} \frac{\overleftarrow{\partial}}{\partial g_{q}}\right]\left[B(\tilde{g}(t)) B^{T}(\tilde{g}(t))\right]_{q p}}
$$

The detailed derivation of this important equation is set out in Appendix 16.

\subsection{Key Result}

By comparing the expressions in (55) and (68) we see that the time derivatives of the stochastic and phase space averages same if relationships between $A$ and $D$ in the Fokker-Planck equation, and $C$ and $B$ occurring in Ito stochastic equation are

$$
\begin{aligned}
C^{p}\left(g, g^{+}\right) & =-A_{p}\left(g, g^{+}\right) \\
{\left[B\left(g, g^{+}\right) B^{T}\left(g, g^{+}\right)\right]_{q p} } & =D_{q p}\left(g, g^{+}\right)
\end{aligned}
$$

If the phase space and stochastic averages are chosen to be equal at an initial time - by choosing the initial stochastic averages of all products of the stochastic Grassmann variables to be the same as the corresponding phase space variables as determined from the $B$ distribution function at the initial time - then the phase space and stochastic averages will remain equal at later times.

In the case of the drift term, we can always choose $C=-A$, so the drift term condition is satisfied Note the minus sign in the relationship in the case of fermions - for bosons there is a plus sign. In the case of the diffusion term we need to show that a matrix $B$ can be found such that $B B^{T}=D$. In the boson case where $B$ and $D$ are c-functions and $D$ is symmetric, with $D^{T}=+D$ the existence of $B$ follows from the Takagi factorisation [48] of complex symmetric matrices (see [49] for proof of the factorisation). In the fermion case $B$ and $D$ are Grassmann functions and $D$ is anti-symmetric with $D^{T}=-D$. The existence of such a matrix $B$ needs to be established. 


\subsection{Solution for Matrix B}

To show that the required matrix $B$ can be found we first write the matrix elements for the sub-matrices (52) of $D$ as

$$
\begin{aligned}
-F_{i j}^{--} & =\sum_{k l} M_{i k j l}^{--} g_{k} g_{l} & F_{i j}^{++} & =\sum_{k l} M_{i k j l}^{++} g_{k}^{+} g_{l}^{+} \\
F_{i j}^{-+} & =\sum_{k l} M_{i k j l}^{-+} g_{k} g_{l}^{+} & F_{i j}^{+-} & =\sum_{k l} M_{i k j l}^{+-} g_{k}^{+} g_{l}
\end{aligned}
$$

where $M^{--}, M^{++}, M^{-+}$and $M^{+-}$are four $n^{2} \times n^{2}$ c-number matrices . From the explicit expressions in (52) we see that $M^{--}, M^{++}$are symmetric and $M^{-+}, M^{+-}$are hermitean.

In fact we can write the diffusion matrix elements in the form

$$
D_{p q}=\sum_{r, s=1}^{2 n} Q_{r s}^{p q} g_{r} g_{s}
$$

where the Grassmann phase space variables $g_{r}$ or $g_{s}$ are now listed $g_{1}, g_{2}, \cdots, g_{n}, g_{1}^{+}, g_{2}^{+}, \cdots, g_{n}^{+}$ as before. The quantity $Q$ is a $2 n^{2} \times 2 n^{2}$ complex and symmetric matrix (from $D_{p q}=-D_{q p}$ ), with its rows listed as $p, r$ and its columns listed as $q, s$. Note that for a given $p, r, q, s$ the elements $Q_{r, s}^{p, q}$ that are non-zero elements are obtained from the following Table

\begin{tabular}{|l|l|l|l|l|}
\hline $\mathbf{g}_{p}$ & $\mathbf{g}_{q}$ & $\mathbf{g}_{r}$ & $\mathbf{g}_{s}$ & $\mathbf{Q}_{r, s}^{p, q}$ \\
\hline$g_{i}$ & $g_{j}$ & $g_{k}$ & $g_{l}$ & $M_{i k}^{-} \overline{j l}$ \\
\hline$g_{i}$ & $g_{j}^{+}$ & $g_{k}$ & $g_{l}^{+}$ & $M_{i k}^{-}+\dot{j l}$ \\
\hline$g_{i}^{+}$ & $g_{j}$ & $g_{k}^{+}$ & $g_{l}$ & $M_{i k}^{+} \overline{j l}$ \\
\hline$g_{i}^{+}$ & $g_{j}^{+}$ & $g_{k}^{+}$ & $g_{l}^{+}$ & $M_{i k}^{+}+$ \\
\hline
\end{tabular}

Note that in each $n^{2} \times n^{2}$ sub-matrix of $Q$, the rows $p, r$ are in one-one correspondence with the various $i, k$ and the columns $q, s$ are in one-one correspondence with the various $j, l$. The $i, k, j, l$ only run from $1,2, \cdots, n$. The reason that $Q$ does not have $(2 n)^{2}=4 n^{2}$ rows or columns is that with the rows given by $p, r$ and columns listed as $q, s$, the rows $g_{p}, g_{r} \equiv g_{i}, g_{k}$ are only associated with columns $g_{q}, g_{s} \equiv g_{j}, g_{l}$ or $g_{q}, g_{s} \equiv g_{j}^{+}, g_{l}^{+}$and not $g_{q}, g_{s} \equiv g_{j}^{+}, g_{l}$ or $g_{q}, g_{s} \equiv g_{j}, g_{l}^{+}$. Similarly the rows $g_{p}, g_{r} \equiv g_{i}^{+}, g_{k}^{+}$are only associated with columns $g_{q}, g_{s} \equiv g_{j}, g_{l}$ or $g_{q}, g_{s} \equiv g_{j}^{+}, g_{l}^{+}$and not $g_{q}, g_{s} \equiv g_{j}^{+}, g_{l}$ or $g_{q}, g_{s} \equiv g_{j}, g_{l}^{+}$.

Using Takagi factorisation [48] we can write the complex symmetric matrix $Q$ as

$$
\begin{aligned}
Q & =K(K)^{T} \\
Q_{r s}^{p q} & =\sum_{a} K_{r, a}^{p} K_{s, a}^{q}
\end{aligned}
$$

where $K$ is a square c-number matrix with the same number of rows or columns as in $Q$. Since $Q$ is $2 n^{2} \times 2 n^{2}$ then $a=1,2, \cdots, 2 n^{2}$ lists the columns of $K$. Note the $K_{r, a}^{p}$ are c-numbers.

Using (70) the expression for $B_{a}^{p}$ in the Ito stochastic equation will be given by

$$
B_{a}^{p}\left(g, g^{+}\right)=\sum_{r=1}^{2 n} K_{r, a}^{p} g_{r}
$$

since we now show that $B B^{T}=D$. For we have

$$
\begin{aligned}
\sum_{a} B_{a}^{p}(g) B_{a}^{q}(g) & =\sum_{a} \sum_{r} K_{r, a}^{p} g_{r} \sum_{s} K_{s, a}^{q} g_{s} \\
& =\sum_{r, s} Q_{r s}^{p q} g_{r} g_{s}=D_{p q}(g)
\end{aligned}
$$


Hence we have now found a matrix $B$ such that $B B^{T}=D$. As there are $2 n^{2}$ columns for $K$ it follows that the number of Gaussian-Markoff or Wiener stochastic variables in the Ito equations is $2 n^{2}$ also. This contrasts to the smaller number $2 n$ for the boson case.

\subsection{Solution for Vector C}

We can also write the solution for $C$ in a more convenient form. We first note that the sub-matrices for the drift vector $(52)$ can be written as

$$
C_{i}^{-}=-\sum_{j} L_{i j}^{-} g_{j} \quad C_{i}^{+}=-\sum_{j} L_{i j}^{+} g_{j}^{+}
$$

Combining the last pair of equations into a single expression we write the drift vector in the form

$$
A_{p}(g)=-\sum_{r} L_{r}^{p} g_{r}
$$

where $L$ is a $2 n \times 2 n$ c-number matrix with rows $p$ and columns $r$ listed as $1,2, \cdots, 2 n$. the matrix $L$ has non-zero elements obtained from the following Table

\begin{tabular}{|l|l|l|}
\hline $\mathbf{g}_{p}$ & $\mathbf{g}_{r}$ & $\mathbf{L}_{r}^{p}$ \\
\hline$g_{i}$ & $g_{j}$ & $L_{i j}^{-}$ \\
\hline$g_{i}^{+}$ & $g_{j}^{+}$ & $L_{i j}^{+}$ \\
\hline
\end{tabular}

Note that many elements of $L$ are zero, for example with row $g_{p}=g_{i}$ there are non zero entries in columns $g_{r}=g_{j}$ but not in columns $g_{r}=g_{j}^{+}$.

Using (69) the expression for $C^{p}$ in the Ito stochastic equation will be given by

$$
C^{p}\left(g, g^{+}\right)=\sum_{r=1}^{2 n} L_{r}^{p} g_{r}
$$

\subsection{Form of Ito Stochastic Equation}

Combining the results for $C^{p}(\tilde{g}(t))$ and $B_{a}^{p}(\tilde{g}(t))$ we can finally write the Ito stochastic equations in the form

$$
\begin{aligned}
\tilde{g}_{p}(t+\delta t) & =\sum_{r}\left\{\delta_{p, r}+L_{r}^{p} \delta t+\sum_{a} K_{r, a}^{p} \delta \tilde{w}_{a}\left(t_{+}\right)\right\} \tilde{g}_{r}(t) \\
& =\sum_{r} \Theta_{p, r}\left(t^{+}\right) \tilde{g}_{r}(t)
\end{aligned}
$$

where $\delta \tilde{w}_{a}\left(t_{+}\right)$is a Wiener increment as in (66) and the c-number stochastic matrix $\Theta_{p, r}\left(t^{+}\right)$is defined in (82).

We see that the quantities in brackets are stochastic c-numbers, shows that $\tilde{g}_{p}(t+\delta t)$ is related linearly to the $\tilde{g}_{r}(t)$ via non-Grassmann quantities $\Theta_{p, r}\left(t^{+}\right)$. This feature is only present for the $B$ distribution - for the $P$ distribution the drift vector involves cubic terms. A feature similar to $\Theta_{p, r}^{-1}\left(t^{+}\right)$is presented in Plimak et al [23], though no detailed proof was provided.

Note that the number of stochastic c-number Wiener increments involved is $2 n^{2}$. This number increases only as the square of number of modes, making numerics possible. A similar number of of Wiener increments also occurs in the Gaussian phase-space treatment developed by Corney and Drummond [18]. 


\subsection{Numerics}

The last equation indicates how numerical calculations could be carried out to determine stochastic Grassmann phase variables at a final time $t_{f}$ as linear combinations of stochastic Grassmann phase variables at an earlier time $t_{0}$ using stochastic quantities that are c-numbers We can divide the period between $t_{0}$ and $t_{f}=t_{n+1}$ into small intervals $t_{i} \rightarrow t_{i+1}(i=0, \cdots, n)$ and a repeated application of (81) gives

$$
\begin{aligned}
\tilde{g}_{p}\left(t_{f}\right) & =\sum_{r, s, \cdots, z} \Theta_{p, r}\left(t_{n}^{+}\right) \Theta_{r, s}\left(t_{n-1}^{+}\right) . . \Theta_{y, z}\left(t_{0}^{+}\right) \tilde{g}_{z}\left(t_{0}\right) \\
& =\sum_{z} \Lambda_{p, z}\left(t_{f}, t_{0}\right) \tilde{g}_{z}\left(t_{0}\right)
\end{aligned}
$$

so the quantity $\Lambda_{p, z}\left(t_{f}, t_{0}\right)$ is a c-number.

Hence the stochastic average of products of the $\tilde{g}_{p}\left(t_{f}\right)$ at time $t_{f}$ will be given by sums over stochastic averages of products of c-number stochastic quantities $\Lambda_{p, z}\left(t_{f}, t_{0}\right)$ times stochastic averages of products of $\tilde{g}_{z}\left(t_{0}\right)$ at time $t_{0}$ - these occuring in the same numbers as for $\tilde{g}_{p}$. The stochastic averages of products of $\tilde{g}_{z}\left(t_{0}\right)$ at time $t_{0}$ are determined from the initial density operator at time $t_{0}$, which can be used to evaluate all the Fock state populations and coherences at $t_{0}$ based on the definitions in (30) and (31). From Eqs. (57) and (57) these are given by stochastic averages of the $\tilde{g}_{z}\left(t_{0}\right)$. Overall, none of the actual numerical calculations involve computations with Grassmann variables, so numerical calculations for dynamical and thermal evolution of fermion systems are possible based on Grassmann phase space theory in its stochastic form.

To apply the key result (83) for the simple case of a large number of equal small intervals $t_{i+1}=t_{i}+\delta t$, the following results for stochastic averages of products of Wiener increments are used.

$$
\begin{aligned}
\overline{\delta \tilde{w}_{a}\left(t_{i}\right)}= & 0 \\
\overline{\delta \tilde{w}_{a}\left(t_{i}\right) \delta \tilde{w}_{b}\left(t_{j}\right)}= & \delta_{a b} \delta_{i j}(\delta t) \\
\overline{\delta \tilde{w}_{a}\left(t_{i}\right) \delta \tilde{w}_{b}\left(t_{j}\right) \delta \tilde{w}_{c}\left(t_{k}\right)}= & 0 \\
\overline{\delta w_{a}\left(t_{i}\right) \delta w_{b}\left(t_{j}\right) \delta w_{c}\left(t_{k}\right) \delta w_{d}\left(t_{l}\right)}= & \overline{\delta \tilde{w}_{a}\left(t_{i}\right) \delta \tilde{w}_{b}\left(t_{j}\right)} \overline{\delta \tilde{w}_{c}\left(t_{k}\right) \delta \tilde{w}_{d}\left(t_{l}\right)}+\overline{\delta \tilde{w}_{a}\left(t_{i}\right) \delta \tilde{w}_{c}\left(t_{k}\right)} \overline{\delta \tilde{w}_{b}\left(t_{j}\right) \delta \tilde{w}_{d}\left(t_{l}\right)} \\
& +\overline{\delta \tilde{w}_{a}\left(t_{i}\right) \delta \tilde{w}_{d}\left(t_{l}\right)} \overline{\delta \tilde{w}_{b}\left(t_{j}\right) \delta \tilde{w}_{c}\left(t_{k}\right)} \\
= & \left\{\delta_{a b} \delta_{i j} \delta_{c d} \delta_{k l}+\delta_{a c} \delta_{i k} \delta_{b d} \delta_{j l}+\delta_{a d} \delta_{i l} \delta_{b c} \delta_{j k}\right\}(\delta t)^{2} \\
& \text { etc }
\end{aligned}
$$

These results are easily derived from (66) and (61).

As an example, we determine the stochastic average $\overline{\tilde{g}_{p}\left(t_{f}\right) \tilde{g}_{q}\left(t_{f}\right)}$ using (83) and (81) for the case of two intervals $t_{1}=t_{0}+\delta t, t_{2}=t_{1}+\delta t=t_{f}$. We find that

$$
\begin{aligned}
& \overline{\tilde{g}_{p}\left(t_{f}\right) \tilde{g}_{q}\left(t_{f}\right)} \\
= & \sum_{r, z} \sum_{s, y}\left[\begin{array}{r}
\left\{\delta_{p, r}+L_{r}^{p} \delta t+\sum_{a} K_{r, a}^{p} \delta \tilde{w}_{a}\left(t_{1+}\right)\right\}\left\{\delta_{q, s}+L_{s}^{q} \delta t+\sum_{b} K_{s, b}^{q} \delta \tilde{w}_{b}\left(t_{1+}\right)\right\} \\
\left.\times\left\{\delta_{r, z}+L_{z}^{r} \delta t+\sum_{c} K_{z, c}^{r} \delta \tilde{w}_{c}\left(t_{0+}\right)\right\}\left\{\delta_{s, y}+L_{y}^{s} \delta t+\sum_{d} K_{y, d}^{s} \delta \tilde{w}_{d}\left(t_{0+}\right)\right\}\right]_{\text {stoch aver }}
\end{array}\right. \\
& \times \frac{\tilde{g}_{z}\left(t_{0}\right) \tilde{g}_{y}\left(t_{0}\right)}{}
\end{aligned}
$$

noting that the c-number quantities $\{\cdots\}$ can be commuted. The quantities $L_{r}^{p}$, $K_{r, a}^{p}$ etc are c-numbers obtained from the drift and diffusion matrices in the Fokker-Planck equations as in 
(78), (72) and (74). The initial stochastic averages $\overline{\tilde{g}_{z}\left(t_{0}\right) \tilde{g}_{y}\left(t_{0}\right)}$ are obtained using (53) and the equivalent expression for the phase space average obtained from the initial density operator via (28). The stochastic averages of products of Wiener increments are given by (85), resulting in the stochastic average $\overline{\tilde{g}_{p}\left(t_{f}\right) \tilde{g}_{q}\left(t_{f}\right)}$ being given to $O\left((\delta t)^{2}\right)$.

We now describe how numerical calculations of the stochastic averages of final stochastic products of the $\tilde{g}_{p}\left(t_{f}\right)$ of a given order might be carried out. We start from Eq. (81) with $t \rightarrow t_{i}$ and $t_{i+1}=t_{i}+\delta t$. Considering a product $\tilde{g}_{p}\left(t_{i}+\delta t\right) \tilde{g}_{q}\left(t_{i}+\delta t\right) \cdots \tilde{g}_{s}\left(t_{i}+\delta t\right)$ of a given order we get

$$
\begin{aligned}
& \tilde{g}_{p}\left(t_{i}+\delta t\right) \tilde{g}_{q}\left(t_{i}+\delta t\right) \cdots \tilde{g}_{s}\left(t_{i}+\delta t\right) \\
= & \sum_{x y \cdots u}\left\{\delta_{p, x}+L_{x}^{p} \delta t+\sum_{a} K_{x, a}^{p} \delta \tilde{w}_{a}\left(t_{i+}\right)\right\} \tilde{g}_{x}\left(t_{i}\right) \\
& \times\left\{\delta_{q, y}+L_{y}^{q} \delta t+\sum_{b} K_{y, b}^{q} \delta \tilde{w}_{b}\left(t_{i+}\right)\right\} \tilde{g}_{y}\left(t_{i}\right) \\
& \times \cdots \\
& \times\left\{\delta_{s, u}+L_{u}^{s} \delta t+\sum_{e} K_{u, e}^{s} \delta \tilde{w}_{e}\left(t_{i+}\right)\right\} \tilde{g}_{u}\left(t_{i}\right)
\end{aligned}
$$

Placing all the stochastic Grassmann variables for time $t_{i}$ together in order and taking the stochastic average of both sides we then find that

$$
\begin{gathered}
\quad \overline{\tilde{g}_{p}\left(t_{i}+\delta t\right) \tilde{g}_{q}\left(t_{i}+\delta t\right) \cdots \tilde{g}_{s}\left(t_{i}+\delta t\right)} \\
=\sum_{x y \cdots u}\left[\begin{array}{c}
\left\{\delta_{p, x}+L_{x}^{p} \delta t+\sum_{a} K_{x, a}^{p} \delta \tilde{w}_{a}\left(t_{i+}\right)\right\} \\
\times\left\{\delta_{q, y}+L_{y}^{q} \delta t+\sum_{b} K_{y, b}^{q} \delta \tilde{w}_{b}\left(t_{i+}\right)\right\} \\
\cdots \\
\times\left\{\delta_{s, u}+L_{u}^{s} \delta t+\sum_{e} K_{u, e}^{s} \delta \tilde{w}_{e}\left(t_{i+}\right)\right\}
\end{array}\right]_{\text {stoch aver }} \\
\times \frac{\left[\tilde{g}_{x}\left(t_{i}\right) \tilde{g}_{y}\left(t_{i}\right) \cdots \tilde{g}_{u}\left(t_{i}\right)\right.}{}
\end{gathered}
$$

using the non-correlation property in Eq. (62). This shows that the stochastic average of a product $\overline{\tilde{g}_{p}\left(t_{i}+\delta t\right) \tilde{g}_{q}\left(t_{i}+\delta t\right) \cdots \tilde{g}_{s}\left(t_{i}+\delta t\right)}$ of a given order at time $t_{i+1}$ can be obtained from the stochastic averages of all the products $\overline{\tilde{g}}_{x}\left(t_{i}\right) \tilde{g}_{y}\left(t_{i}\right) \cdots \tilde{g}_{u}\left(t_{i}\right)$ of the same order at time $t_{i}$ via the stochastic average of a c-number sum involving all the $2 n^{2}$ Wiener increments $\delta \tilde{w}_{a}\left(t_{i+}\right)$ at time $t_{i}$ along with quantities such as the $L_{x}^{p}$ and the $K_{x, a}^{p}$ that are obtained from the drift and diffusion terms in the Fokker-Planck equation. Thus, the process of determining the stochasic averages of products of the stochastic Grassmann variables at the later time $t_{i+1}=t_{i}+\delta t$ from those at earlier time $t_{i}$ only involves c-numbers - which overcomes one of the objections to using Grassmann distribution functions.

Applied further, the result (88) would enable such as stochastic average $\overline{\tilde{g}_{p}\left(t_{f}\right) \tilde{g}_{q}\left(t_{f}\right) \cdots \tilde{g}_{s}\left(t_{f}\right)}$ at final time $t_{f}$ to be determined in steps starting from the stochastic averages $\frac{\tilde{g}_{x}\left(t_{0}\right) \tilde{g}_{y}\left(t_{0}\right) \cdots \tilde{g}_{u}\left(t_{0}\right)}{t_{0}}$ at initial time $t_{0}$. The latter are obtained as before from the initial density operator. This approach would reduce the computer storage requirements, as intermediate sets of stochastic aver-

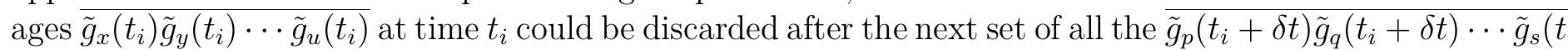
at time $t_{i+1}$ has been calculated. Furthermore, the Wiener increments for each time point $t_{i}$ would only be required for that time point, so sets of Wiener increments for all the time points need not be stored at once - again reducing computer storage requirements. We believe that a numerical approach based on Eq. (88) would enable calculations of quantum correlation functions, Fock state populations and coherences to be carried out without the need to represent the Grassmann phase space variables on the computer. 


\subsection{Case of $P$ Distribution}

In the case of the $P$ distribution the Fokker-Planck equation is set out as (214) in Appendix 15 and has the same general features as that for the $B$ distribution, the key difference being that the drift term involves cubic functions of the Grassmann variables. Ito stochastic equations also apply for the $P$ distribution, and these also are set out as (218) in Appendix 15. As the diffusion matrix is unchanged, the same solution for the noise term $B_{a}^{p}$ as in (75) still applies. However for the classical term $C^{p}$ now depends cubically on the Grassmann variables, so the linear relation (81) between $\tilde{g}_{p}(t+\delta t)$ and the $\tilde{g}_{r}(t)$ no longer applies. Hence there is also no linear relation (83) just involving c-numbers between stochastic Grassmann phase variables at a final time $t_{f}$ and stochastic Grassmann phase variables at an earlier time $t_{0}$, so numerical calculations based on the $P$ distribution version of Grassmann phase space theory do not seem possible. It was of course the insight of Plimak, Collett and Olsen [23] to use the $B$ distribution that enables numerical work to be carried out.

\section{Application to Fermion Mode Model}

\subsection{Model}

As an illustration of the results we consider a simple four mode model showing onset of coupling between Cooper pair states due to spin conserving collisions between pairs of spin 1/2 fermionic atoms. The atoms are untrapped and relaxation is ignored. A field operator version of this system was considered by Plimak et al [23] using Matsubara equations [46] to determine the evolution of correlations between states with momenta $-\mathbf{k},+\mathbf{k}$ and spin components,-+ as the temperature was lowered with the fermion system remaining in thermal equilibrium the density operator being that for the grand canonical ensemble. Four peaks were seen in the correlation function $\left\langle\hat{n}_{\mathbf{m},+}, \hat{n}_{\mathbf{p},-}\right\rangle=\left\langle\hat{n}_{\mathbf{m},+} \hat{n}_{\mathbf{p},-}\right\rangle-\left\langle\hat{n}_{\mathbf{m},+}\right\rangle\left\langle\hat{n}_{\mathbf{p},-}\right\rangle$ around momentum pairs $\{\mathbf{m}, \mathbf{p}\}=\{\mathbf{k},-\mathbf{k}\},\{-\mathbf{k}, \mathbf{k}\},\{\mathbf{k}, \mathbf{k}\}$ and $\{-\mathbf{k},-\mathbf{k}\}$. The first two peaks are expected as they correspond to two Cooper pair states $|\mathbf{k},+;-\mathbf{k},-\rangle$ and $|\mathbf{k},-;-\mathbf{k},+\rangle$ in which pairs of fermions with opposite momenta are involved, but the last two are anomalous since Cooper pairs do not involve fermions with the same momenta. Since Matsubara equations are associated with energy eigenstates and the two distinct Cooper pair states would be degenerate unless there is coupling between the fermions, the presenceof the anomalous peaks indicates there must be coupling between the two Cooper pair states. As we will see, the spin conserving collisions between pairs of spin $1 / 2$ fermionic atoms can account for this coupling, and its effects would also be seen in the creation of coherence between the two Cooper pair states if the system was initially prepared in one of the Cooper pair states. We will now determine the short time evolution of the coherence to illustrate the Grassmann phase space approach via solving the Ito stochastic equations.

The modes involved are free space modes

$$
\left|\phi_{1}\right\rangle=\left|\phi_{\mathbf{k},+}\right\rangle \quad\left|\phi_{2}\right\rangle=\left|\phi_{\mathbf{k},-}\right\rangle \quad\left|\phi_{3}\right\rangle=\left|\phi_{-\mathbf{k},+}\right\rangle \quad\left|\phi_{4}\right\rangle=\left|\phi_{-\mathbf{k},-}\right\rangle
$$

with momenta $-\mathbf{k},+\mathbf{k}$ and spin components,-+ . The spatial mode functions are plane waves $\exp ( \pm i \mathbf{k} \cdot \mathbf{r})$, normalised in a box of volume $V$.

The Hamiltonian is given in (38). A straight-forward evaluation of the Hamiltonian parameters 
in Eqs.(39) gives

$$
\begin{aligned}
h_{11} & =h_{22}=h_{33}=h_{44}=\hbar \omega \\
\nu_{1212} & =\nu_{2121}=\nu_{1414}=\nu_{4141}=\nu_{3232}=\nu_{2323}=\nu_{3434}=\nu_{4343}=\kappa \\
\nu_{1432} & =\nu_{4123}=\nu_{3214}=\nu_{2341}=\kappa
\end{aligned}
$$

with $\hbar \omega=\hbar^{2} k^{2} / 2 m$ and $\kappa=g / V$. All other one and two body matrix elements are zero.

\subsection{Initial Conditions}

For the case of $N=2$ fermions there are six different Fock states, designated $\left|\Phi_{a}\right\rangle$

$$
\begin{aligned}
& \left|\Phi_{1}\right\rangle=\hat{c}_{1}^{\dagger} \hat{c}_{2}^{\dagger}|0\rangle \quad\left|\Phi_{2}\right\rangle=\hat{c}_{3}^{\dagger} \hat{c}_{4}^{\dagger}|0\rangle \quad\left|\Phi_{3}\right\rangle=\hat{c}_{1}^{\dagger} \hat{c}_{4}^{\dagger}|0\rangle \quad\left|\Phi_{4}\right\rangle=\hat{c}_{2}^{\dagger} \hat{c}_{3}^{\dagger}|0\rangle \\
& \left|\Phi_{5}\right\rangle=\hat{c}_{1}^{\dagger} \hat{c}_{3}^{\dagger}|0\rangle \quad\left|\Phi_{6}\right\rangle=\hat{c}_{2}^{\dagger} \hat{c}_{4}^{\dagger}|0\rangle
\end{aligned}
$$

The states $\left|\Phi_{1}\right\rangle$ to $\left|\Phi_{4}\right\rangle$ are non-magnetic, having one fermion in a spin + mode and one fermion in a spin - mode. States $\left|\Phi_{5}\right\rangle$ and $\left|\Phi_{6}\right\rangle$ are magnetic, with both fermions in either a spin + mode or a spin - mode. The states $\left|\Phi_{3}\right\rangle$ and $\left|\Phi_{4}\right\rangle$ are Cooper pair type states, each having one fermion with momentum $+\mathbf{k}$ and the other with momentum $-\mathbf{k}$. These have the same energy if fermion-fermion interactions are ignored.

We consider the case where the initial state is the pure Cooper pair state $\left|\Phi_{3}\right\rangle$

$$
\hat{\rho}(0)=\left|\Phi_{3}\right\rangle\left\langle\Phi_{3}\right|
$$

with one fermion in mode $\phi_{\mathbf{k}(+)}$ the other in mode $\phi_{-\mathbf{k}(-)}$.

The interaction term $\nu_{1432} \hat{c}_{3}^{\dagger} \hat{c}_{2}^{\dagger} \hat{c}_{4} \hat{c}_{1}$ couples the Cooper pair state $\left|\Phi_{3}\right\rangle$ to the other Cooper pair state $\left|\Phi_{4}\right\rangle$, which has one fermion in the mode $\phi_{\mathbf{k}(-)}$ and the other in mode $\phi_{-\mathbf{k}(+)}$, so a non-zero coherence between state $\left|\Phi_{3}\right\rangle$ and state $\left|\Phi_{4}\right\rangle$ should develop. We will determine this coherence, whose presence indicates coupling between Cooper pair states each with two fermions with opposite momenta and spins is taking place.

\subsection{Ito Stochastic Equations}

The Ito equations are obtained from the general result (81), after determining the $L_{r}^{p}$ and $K_{r, a}^{p}$ from (80) and (74), having first obtained the drift vector and diffusion matrices from (50), (51) and (52) using the parameters in (90) and then identifying the $L$ and $Q$ matrices via Tables (79) and (73).

The Ito equations are

$$
\begin{aligned}
& \tilde{g}_{1}(t+\delta t)=\left\{(1-i \omega \delta t)+\quad \frac{\lambda}{\sqrt{2}}\left(\delta \tilde{w}_{1}+\delta \tilde{w}_{11}\right)\right\} \tilde{g}_{1}(t)+\left\{\frac{\lambda}{\sqrt{2}}\left(\delta \tilde{w}_{3}+\delta \tilde{w}_{14}\right)\right\} \tilde{g}_{3}(t) \\
& \tilde{g}_{2}(t+\delta t)=\left\{(1-i \omega \delta t)+\frac{\lambda}{\sqrt{2}}\left(\delta \tilde{w}_{6}+\delta \tilde{w}_{16}\right)\right\} \tilde{g}_{2}(t)+\left\{\frac{\lambda}{\sqrt{2}}\left(\delta \tilde{w}_{8}+\delta \tilde{w}_{9}\right)\right\} \tilde{g}_{4}(t) \\
& \tilde{g}_{3}(t+\delta t)=\left\{(1-i \omega \delta t)+\quad \frac{\lambda}{\sqrt{2}}\left(\delta \tilde{w}_{1}+\delta \tilde{w}_{11}\right)\right\} \tilde{g}_{3}(t)+\left\{\frac{\lambda}{\sqrt{2}}\left(\delta \tilde{w}_{8}+\delta \tilde{w}_{9}\right)\right\} \tilde{g}_{1}(t) \\
& \tilde{g}_{4}(t+\delta t)=\left\{(1-i \omega \delta t)+\quad \frac{\lambda}{\sqrt{2}}\left(\delta \tilde{w}_{6}+\delta \tilde{w}_{16}\right)\right\} \tilde{g}_{4}(t)+\left\{\frac{\lambda}{\sqrt{2}}\left(\delta \tilde{w}_{3}+\delta \tilde{w}_{14}\right)\right\} \tilde{g}_{2}(t)
\end{aligned}
$$


and

$$
\begin{array}{ll}
\tilde{g}_{1}^{+}(t+\delta t)=\{(1+i \omega \delta t)+ & \left.\frac{\lambda^{*}}{\sqrt{2}}\left(\delta \tilde{w}_{1}^{+}+\delta \tilde{w}_{11}^{+}\right)\right\} \tilde{g}_{1}^{+}(t)+\left\{\frac{\lambda^{*}}{\sqrt{2}}\left(\delta \tilde{w}_{3}^{+}+\delta \tilde{w}_{14}^{+}\right)\right\} \tilde{g}_{3}^{+}(t) \\
\tilde{g}_{2}^{+}(t+\delta t)=\{(1+i \omega \delta t)+ & \left.\frac{\lambda^{*}}{\sqrt{2}}\left(\delta \tilde{w}_{6}^{+}+\delta \tilde{w}_{16}^{+}\right)\right\} \tilde{g}_{2}^{+}(t)+\left\{\frac{\lambda^{*}}{\sqrt{2}}\left(\delta \tilde{w}_{8}^{+}+\delta \tilde{w}_{9}^{+}\right)\right\} \tilde{g}_{4}^{+}(t) \\
\tilde{g}_{3}^{+}(t+\delta t)=\{(1+i \omega \delta t)+ & \left.\frac{\lambda^{*}}{\sqrt{2}}\left(\delta \tilde{w}_{1}^{+}+\delta \tilde{w}_{11}^{+}\right)\right\} \tilde{g}_{3}^{+}(t)+\left\{\frac{\lambda^{*}}{\sqrt{2}}\left(\delta \tilde{w}_{8}^{+}+\delta \tilde{w}_{9}^{+}\right)\right\} \tilde{g}_{1}^{+}(t) \\
\tilde{g}_{4}^{+}(t+\delta t)=\{(1+i \omega \delta t)+ & \left.\frac{\lambda^{*}}{\sqrt{2}}\left(\delta \tilde{w}_{6}^{+}+\delta \tilde{w}_{16}^{+}\right)\right\} \tilde{g}_{4}^{+}(t)+\left\{\frac{\lambda^{*}}{\sqrt{2}}\left(\delta \tilde{w}_{3}^{+}+\delta \tilde{w}_{14}^{+}\right)\right\} \tilde{g}_{2}^{+}(t)
\end{array}
$$

where $\lambda=\kappa / i \hbar$. Because of there being no cross-coupling between the $\tilde{g}_{i}$ and the $\tilde{g}_{j}^{+}$in the diffusion matrix, the overall number of Wiener increments involved is 16, which is less than the expected number $2 n^{2}=32$. The Wiener increments have been numbered $1,2, \cdots, 16$.

\subsection{Populations and Coherences}

Using (57) and (58) the population for Fock state $\left|\Phi_{3}\right\rangle$, and coherence between Fock states $\left|\Phi_{3}\right\rangle$ and $\left|\Phi_{4}\right\rangle$ are given by

$$
\begin{aligned}
P\left(\Phi_{3}\right) & =\overline{\tilde{g}_{4} \tilde{g}_{1} \tilde{g}_{1}^{+} \tilde{g}_{4}^{+}} \\
C\left(\Phi_{4} ; \Phi_{3}\right) & =\frac{\tilde{g}_{4} \tilde{g}_{1} \tilde{g}_{2}^{+} \tilde{g}_{3}^{+}}{}
\end{aligned}
$$

From the initial condition the population of Fock states $\left|\Phi_{3}\right\rangle$ equal to unity. Hence from (95)

$$
\left(\overline{\tilde{g}_{4} \tilde{g}_{1} \tilde{g}_{1}^{+} \tilde{g}_{4}^{+}}\right)_{t=0}=1=-\left(\overline{\tilde{g}_{4} \tilde{g}_{1} \tilde{g}_{4}^{+} \tilde{g}_{1}^{+}}\right)_{t=0}
$$

All other initial stochastic averages are zero.

To determine the coherence $C\left(\Phi_{4} ; \Phi_{3}\right)$ at time $\delta t$ we use (93) and (94) to substitute for $\tilde{g}_{4}(t+\delta t), \tilde{g}_{1}(t+\delta t), \tilde{g}_{2}^{+}(t+\delta t)$ and $\tilde{g}_{3}^{+}(t+\delta t)$. The result involves product of four factors each containiing two terms. When multiplied out there are sixteen terms, each the product of two $\tilde{g}_{i}(0)$, two $\tilde{g}_{j}^{+}(0)$ and factors involving Wiener increments. When the stochastic average is taken, the stochastic average for each of the sixteen terms factorises into a stochastic average of the product of the two $\tilde{g}_{i}(0)$ and two $\tilde{g}_{j}^{+}(0)$ and the stochastic average of the factors involving the Wiener increments. The only non-zero stochastic average of the two $\tilde{g}_{i}(0)$ and two $\tilde{g}_{j}^{+}(0)$ is $\left(\overline{\tilde{g}_{4} \tilde{g}_{1} \tilde{g}_{1}^{+} \tilde{g}_{4}^{+}}\right)_{t=0}=1$.

This leaves

$$
\begin{aligned}
C\left(\Phi_{4} ; \Phi_{3}\right)_{t=\delta t} & =\left[\left\{(1-i \omega \delta t)+\frac{\lambda}{\sqrt{2}}\left(\delta \tilde{w}_{6}+\delta \tilde{w}_{16}\right)\right\}\left\{(1-i \omega \delta t)+\frac{\lambda}{\sqrt{2}}\left(\delta \tilde{w}_{1}+\delta \tilde{w}_{11}\right)\right\}\right. \\
& \left.\times\left\{\frac{\lambda^{*}}{\sqrt{2}}\left(\delta \tilde{w}_{8}^{+}+\delta \tilde{w}_{9}^{+}\right)\right\}\left\{\frac{\lambda^{*}}{\sqrt{2}}\left(\delta \tilde{w}_{8}^{+}+\delta \tilde{w}_{9}^{+}\right)\right\}\right]_{\text {stoch aver }} \times\left(\overline{\tilde{g}_{4} \tilde{g}_{1} \tilde{g}_{4}^{+} \tilde{g}_{1}^{+}}\right)_{t=0} .
\end{aligned}
$$

We now have terms in the square bracket [..] involving stochastic average of products of two, three and four Wiener increments. Those involving three increments are always zero. Those involving four increments are sums of products of stochastic averages of two Wiener increments. 
These turn out to be zero because the stochastic averages of the particular pairs of Wiener

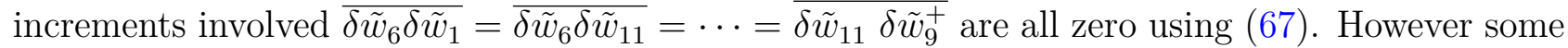
of the products involving just two Wiener increments give a non-zero contribution. These arise from products involving $\delta \tilde{w}_{8}^{+} \delta \tilde{w}_{8}^{+}$and $\delta \tilde{w}_{9}^{+} \delta \tilde{w}_{9}^{+}$in the last two factors and two $(1-i \omega \delta t)$ from the first two factors. Using (67) we see that the non-zero contributions are from

$$
\overline{\delta \tilde{w}_{8}^{+} \delta \tilde{w}_{8}^{+}}=\overline{\delta \tilde{w}_{9}^{+} \delta \tilde{w}_{9}^{+}}=\delta t
$$

We finally obtain the coherence $C\left(\Phi_{4} ; \Phi_{3}\right)$ at time $\delta t$

$$
C\left(\Phi_{4} ; \Phi_{3}\right)_{t=\delta t}=(g / i \hbar V) \delta t
$$

correct to order $\delta t$. This result denonstrates the onset of coherence between two Cooper pair states, one with a fermion in each of $\phi_{\mathbf{k},+}, \phi_{-\mathbf{k},-}$ and other with a fermion in each of $\phi_{\mathbf{k},-}, \phi_{-\mathbf{k},+}$.

\section{Comparison to Other Theoretical Approach}

In a key theory paper applying Grassmann phase space theory to fermions Plimak, Collet and Olsen [23] treated thermal evolution using a Grassmann phase space $B$ distribution based on the Bargmann fermion coherent states, and presented numerical calculations associated with a Matsubara equation. However, our work is based on a different theoretical approach even though we also used the $B$ distribution instead of the more well-known $P$ distribution. Plimak, Collet and Olsen [23] had clearly identified using the $B$ distribution as being vital for treating fermions using a Grassmann phase space distribution - in their words: "For fermions, the presence of product terms (arising from the $P$ distribution) spells disaster: Equation (70) mixes different powers of the algebra generators and hence cannot, in principle, be solved by a linear transformation of the generators." This would not of course make a Grassmann phase space theory necessarily unusable - for example for analytical purposes. In an earlier paper ([24]) for example, we successfully treated the Jaynes-Cummings model via an analytical approach based on a $P$-type Grassmann distribution function. But the real importance of having linear Ito stochastic equations for the Grassmann stochastic phase space variables occurs for numerical work - as we have emphasised in Section 5 in our paper,.

In applying such stochastic methods (both for analytical and numerical work) it is vital to derive the correct Ito stochastic equations for stochastic phase space variables so that they give identical results to those which would be obtained from the Fokker-Planck equation for the $B$ distribution function, when these equivalent approaches are applied to determining quantum correlation functions or Fock state populations and coherences. As discussed in Section 5,our approach actually starts from the physically based requirement that any quantum correlation function, Fock state population etc should be the same whether determined by solving the Fokker-Planck equation over all of phase space and then computing the phase space average of the appropriate products of phase space variables, or alternatively by stochastically averaging over the same products of what are now stochastic phase space variables. We established the link between the Ito stochastic equations and the Fokker-Planck equation via a standard method - originally applied for bosonic systems (see Gardiner, [47], pp 95 -96) - of requiring the phase space average of any arbitrary function of the phase space variables to be the same as the stochastic average of the same function when the phase space variables are replaced by stochastic variables. This leads to a relationship between the terms in the Ito stochastic equations for the latter and the drift and diffusion matrices in the Fokker-Planck equation for the distribution function. For separate modes this is clearly set out in equations (53), (69), 
(70), with the details covered in Section 5 and Appendix 16. Thus, our basic equation (53) for determining the Ito stochastic equations approach has the feature of being directly focused on the stochastic average of typical functions needed for determining quantum correlation functions. In this regard, our theoretical approach is different to that of [23], and as a consequence the Ito stochastic equations we obtain are not equivalent to those obtained in [23].

Although the approach of Plimak et al [23] also involved stochastic Grassmann variables and the $B$ distribution function, their approach to determining Ito stochastic equations was based on a different principle, focused on matching the evolution of the distribution function itself. Their approach is also quite general, applying to bothlinear and non-linear fermionic interactions. As may be seen in their equations (72), (73) they consider an ansatz requiring the distribution function $B\left(g, g^{*}, t\right)$ itself at a final (imaginary) time $t$ to be given by taking the distribution function $B\left(g, g^{*}, 0\right)$ at the initial time 0 , replacing the phase variables $g_{p}$ by time dependent stochastic variables $\tilde{g}_{p}(t)$ and then taking a stochastic average of the stochastic distribution function $B\left(\tilde{g}, g^{*}, 0\right)$ multiplied by the determinent $\operatorname{Det} \beta(t)$ associated with the stochastic c-number linear transformation (101) $\beta^{-1}$ that in matrix form relates the stochastic variables $\tilde{g}_{p}(t)$ to the original (time independent) phase variables $g_{p}$.

$$
\tilde{g}(t)=\beta^{-1} g
$$

where $g \equiv\left\{g_{p}\right\} \equiv\left\{g_{i}, g_{i}^{+}\right\}$(non-stochastic) and $\tilde{g}(t) \equiv\left\{\tilde{g}_{p}(t)\right\} \equiv\left\{\tilde{g}_{i}(t), \tilde{g}_{i}^{+}(t)\right\}$ (stochastic). Their Ito stochastic equations (74) applies to the c-number transformation matrix $\beta$ rather than the $\tilde{g}(t)$, and are of the form

$$
\delta \beta=-m \beta \delta t+\delta Q \beta
$$

where $m$ is a matrix and $\delta Q$ is a matrix whose elements are linear combinations of (Wiener) increments. For the thermal evolution they considered, the complex conjugate phase variables $g_{p}^{*}$ do not become stochastic, though this would be the case if real time evolution had been considered. As indicated in [23], their approach can also be applied to time evolution problems, so if we now consider a distribution function of the form $B\left(g, g^{+}, t\right)$, the ansatz of Plimak et al would lead to

$$
B^{\#}\left(g, g^{+}, t\right)=\overline{\operatorname{Det} \beta(t) B\left(\tilde{g}(t), \tilde{g}^{+}(t), 0\right)}
$$

where the right hand side is a Grassmann function of the phase space variables $g, g^{+}$after the stochastic average is carried out. We denote the distribution function obtained via the Plimak et al ansatz as $B^{\#}\left(g, g^{+}, t\right)$. Their approach involved choosing the matrices $m$ and $\delta Q$ so that the corresponding equation for $B^{\#}\left(g, g^{+}, t\right)$ would match the Fokker-Planck equation for the $B\left(g, g^{+}, t\right)$ distribution function, such as in their Eq. (59). This requirement is stated formally in their Eq. (83), whose form indicates that whilst $m$ is related to the drift term, the stochastic average $\overline{\delta Q^{2}}$ is related to both the drift and diffusion terms. In linking their Ito equation (102) to Fokker-Planck equation, Plimak et al do not provide explicit expressions for $m$ and $\delta Q$ in terms of the drift vector and diffusion matrix along the lines of our Eqs. (69) and (70). This is unfortunate, since if such expressions had been provided it might have been possible to confirm whether $B^{\#}\left(g, g^{+}, t\right)$ does in fact satisfy the Fokker-Planck equation. This key result should be established to validate their ansatz. Plimak et al [23] also derive a stochastic expression (96) involving $\beta$ for the normally ordered characteristic function, from which quantum correlation functions etc could be determined. The reason why (96) does not seem to depend on $B\left(g, g^{*}, 0\right)$ is that for the imaginary time problem considered in [23], the initial imaginary time corresponded to a situation of infinite temperature - where the initial distribution function is fixed by (7) via $R(0)=1$. Our approach for time dependent problems leads to expresssions such as (86) and (98) for typical quantum correlation functions, in which the initial state dependence is reflected in initial stochastic averages of products of Grassmann stochastic variables. As well as not 
proving that their $B^{\#}\left(g, g^{+}, t\right)$ actually satisfies the Fokker-Planck equation, Plimak et al do not provide a proof that the phase space average of the product of typical Grassmann functions $F\left(g, g^{+}\right)$with their $B^{\#}\left(g, g^{+}, t\right)$ gives the same result as the stochastic average of $F\left(\tilde{g}(t), \tilde{g}^{+}(t)\right)$ (which in their case would involve stochastic averages over $\beta$ ) - as is needed to verify the method for calculating quantum correlation functions. It may be possible to provide such proofs, but in view of the lack of detail in [23] regarding the key matrices $m$ and $\delta Q$ this cannot be done here.

The reason why it was thought that the ansatz (103) together with the transformation (101) and a stochastic equation of the form (102) would determine the $B\left(g, g^{+}, t\right)$ distribution function is unclear to us. As described in Appendix 10 (see (157) and (165)), a linear transformation of the form $g=\beta(t) h(t)$ (where initially the c-number matrix $\beta$ is non-stochastic) shows that a phase space integral of the form $\int d g F(g) B(g, t)$ (where $F(g)$ is a Grassmann function associated with a quantum correlation function (or Fock state population) and $B(g, t)$ is the distribution function at time $t)$ is the same as $\left(\operatorname{det}(\beta(t))^{-1} \int d h(t) F(g(h(t)) B(g(h(t)), t)\right.$. If $\left(\operatorname{det}(\beta(t))^{-1} B(g(h(t)), t)\right.$ can be shown to equal $B(h(t), 0)$ (as in (103) before stochastically averaging) then $\int d g F(g) B(g, t)=\int d h(t) F(g(h(t)) B(h(t), 0)$ would express the original phase space average in a form involving the initial distribution function, but now with the original Grassmann variables $g$ replaced by time dependent Grassmann variables $h(t)$, along with similar replacements within $F(g) \rightarrow F(g(h(t))$. It might then be possible to replace $\beta(t)$ by an ensemble of stochastic matrices and then $h(t) \rightarrow \tilde{g}(t)$ becomes an ensemble of stochastic phase space variables, with the phase space average of $F(g(h(t))$ becoming a stochastic average. This may provide some insight into the reasons why Plimak et al chose their ansatz, but as they did not elaborate we can only be speculative. Note also that we need to first show that a solution of the Fokker-Planck equation for $B(g, t)$ can be obtained in the form $B(g, t)=\operatorname{det}(\beta(t)) B(h(t), 0)$. However, we are unaware of any proof that a solution to the Fokker-Planck equation (49) can be obtained in this form based on the initial distribution function and using the linear transformation $g=\beta(t) h(t)$ involving the c-number matrix $\beta$, even for the case where the drift vector, diffusion matrix depend linearly, bilinearly on the Grassmann phase space variables. The further step of making $\beta(t)$ and $h(t) \equiv \tilde{g}(t)$ stochastic and the carrying out a stochastic average $\overline{\operatorname{det}(\beta(t)) B(\tilde{g}(t), 0)}$ in accord with the ansatz requires an additional justification.

Rather than trying to justify the Plimak et al ansatz, we now consider the alternative issue of whether our results are equivalent to those of Plimak et al in spite of the fundamentally different approaches for obtaining the Ito equations, as embodied in our Eq. (53) and their Eq. (103). If our approach and that of Plimak et al are equivalent, then certain derivable quantities or equations should be the same. The matrix $\beta(t)^{-1}$ is equivalent to $\Lambda(t, 0)$ in $(84)$, so it should be possible to derive an Ito stochastic equation for $\beta$ based on our Ito equations. This could be compared to (102). Also, the Plimak et al ansatz could be used in conjunction with our Ito equations to derive an expression for the $B$ distribution function at time $\delta t$. This could be compared to that obtained directly from the Fokker-Planck equation. As we will see, these results from the two approaches do not coincide, so we conclude that our approach and that of Plimak et al are different.

We first examine whether our Ito stochastic equations (81) for the $\tilde{g}_{p}$ are equivalent to their Ito equations (102) for $\beta$. Both reflect the same feature that the stochastic Grassmann phase variables at a later time are related linearly to such variables at an earlier time via a transformation matrix whose elements are c-numbers but also involve stochastic Wiener increments. From (101) we have $\beta(t+\delta t) \tilde{g}(t+\delta t)=\beta(t) \tilde{g}(t)=g$. Then with $\delta \beta(t)=\beta(t+\delta t)-\beta(t)$ and $\delta \tilde{g}(t)=\tilde{g}(t+\delta t)-\tilde{g}(t)$ we have

$$
\delta \beta(t) \tilde{g}(t)+\beta(t) \delta \tilde{g}(t)=0
$$

to first order in $\delta \beta(t)$ and $\delta \tilde{g}(t)$. Substituting for $\delta \tilde{g}(t)$ from our result (81) we get the following 
Grassmann function

$$
\sum_{r} \delta \beta(t)_{p r} \tilde{g}_{r}(t)+\sum_{q} \beta(t)_{p q} \sum_{r}\left\{L_{r}^{q} \delta t+\sum_{a} K_{r, a}^{q} \delta \tilde{w}_{a}\left(t_{+}\right)\right\} \tilde{g}_{r}(t)=0
$$

written in terms of matrix elements. Substituting $\tilde{g}_{r}(t)=\sum_{s}\left(\beta^{-1}(t)\right)_{r s} g_{s}$, then carrying out left integration $\int d g_{s}$ for a specific $s$ and using (141) gives

$$
\delta \beta(t)_{p r}=-\sum_{q} \beta(t)_{p q}\left\{L_{r}^{q} \delta t+\sum_{a} K_{r, a}^{q} \delta \tilde{w}_{a}\left(t_{+}\right)\right\}
$$

after eliminating the common factors $\left(\beta^{-1}\right)_{r s}$. This is of the form

$$
\delta \beta=-\beta n \delta t+\beta \delta R
$$

where $n_{q r}=L_{r}^{q}$ and $\delta R_{q r}=-\sum_{a} K_{r, a}^{q} \delta \tilde{w}_{a}\left(t_{+}\right)$. Although both $n$ and $\delta R$ have the same general features as $m$ and $\delta Q$ in Plimak et al [23], we see the form (107) for the corresponding Ito stochastic equation for the c-number matrix $\beta$ is different to that of Plimak et al in (102) - the matrices $\beta, n$ and $\beta, \delta R$ do not commute. Thus our approach and that of Plimak et al differ in the Ito stochastic equation for the same matrix $\beta$. Hence we may conclude that the approach of Plimak et al must be associated with Ito equations for the $\tilde{g}(t)$ that are different to ours.

Next, we ask whether the distribution function $B^{\#}\left(g, g^{+}, t\right)$ obtained at time $t=\delta t$ via the ansatz (103) of Plimak et al, but where the $\tilde{g}_{p}(\delta t)$ are given by our Ito stochastic equations (60) (governed by (69), (70) linking them to the Fokker-Planck equation), would be the same as the distribution function $B\left(g, g^{+}, t\right)$ obtained directly at time $t=\delta t$ from the Fokker-Planck equation (49). For convenience we calculate in both cases the changes in the distribution function from the common $B\left(g, g^{+}, 0\right)$. From the Fokker-Planck equation we have

$$
\begin{aligned}
\delta B\left(g, g^{+}, 0\right)= & B\left(g, g^{+}, \delta t\right)-B\left(g, g^{+}, 0\right)=\left(\frac{\partial}{\partial t} B\left(g, g^{+}, t\right)\right)_{0} \delta t \\
= & +\sum_{p}\left(B\left(g, g^{+}, 0\right) \frac{\overleftarrow{\partial}}{\partial g_{p}}\right) A_{p} \delta t-B\left(g, g^{+}, 0\right) \sum_{p}\left(A_{p} \frac{\overleftarrow{\partial}}{\partial g_{p}}\right) \delta t \\
& +\frac{1}{2} \sum_{p q}\left(B\left(g, g^{+}, 0\right) \frac{\overleftarrow{\partial}}{\partial g_{q}} \frac{\overleftarrow{\partial}}{\partial g_{p}}\right) D_{p q} \delta t+\frac{1}{2} B\left(g, g^{+}, 0\right) \sum_{p q}\left(D_{p q} \frac{\overleftarrow{\partial}}{\partial g_{q}} \frac{\overleftarrow{\partial}}{\partial g_{p}}\right) \delta t \\
& +\sum_{p q}\left(B\left(g, g^{+}, 0\right) \frac{\overleftarrow{\partial}}{\partial g_{q}}\right)\left(D_{p q} \frac{\overleftarrow{\partial}}{\partial g_{p}}\right) \delta t
\end{aligned}
$$

after using (49) and carrying out Grassmann differentiations. Note that this expression gives the actual change $i$ n the distributiion function over the interval $(0, \delta t)$. To apply the ansatz of Plimak et al we first consider the change to the distribution function $B\left(g, g^{+}, 0\right)$ when the phase space variables are changed from $g, g^{+}$to $g+\delta g, g^{+}+\delta g^{+}$. This change $\delta B^{(+)}\left(g, g^{+}, 0\right)$ is given by

$$
\begin{aligned}
\delta B^{(+)}\left(g, g^{+}, 0\right) & =B\left(g+\delta g, g^{+}+\delta g^{+}, 0\right)-B\left(g, g^{+}, 0\right) \\
& =\sum_{p}\left(B\left(g, g^{+}, 0\right) \frac{\overleftarrow{\partial}}{\partial g_{p}}\right) \delta g_{p}+\frac{1}{2} \sum_{p q}\left(B\left(g, g^{+}, 0\right) \frac{\overleftarrow{\partial}}{\partial g_{q}} \frac{\overleftarrow{\partial}}{\partial g_{p}}\right) \delta g_{p} \delta g_{q}
\end{aligned}
$$


to second order in $\delta g_{p}$. We will then replace $g+\delta g, g^{+}+\delta g^{+}$by the stochastic variables $\tilde{g}(\delta t), \tilde{g}^{+}(\delta t)$, so $\delta g_{p}$ will be replaced by the stochastic quantity $\delta \tilde{g}_{p}(0)=\tilde{g}_{p}(\delta t)-\tilde{g}_{p}(0)=$ $\tilde{g}_{p}(\delta t)-g_{p}$. In order to see whether our Ito stochastic equations produces the same change $\delta B\left(g, g^{+}, 0\right)$ in the distribution function that we obtained in (108), we will substitute

$$
\delta \tilde{g}_{p}(0)=C^{p}\left(g_{q}\right) \delta t+\sum_{a} B_{a}^{p}\left(g_{q}\right) \delta \tilde{w}_{a}\left(0_{+}\right)
$$

from (64) into (109). However, we also need an expression for $\operatorname{Det} \beta(\delta t)$, since before stochastically averaging the required change in the Plimak et al distribution function is

$$
\begin{aligned}
\delta B^{\#}\left(g, g^{+}, 0\right) & =\operatorname{Det} \beta(\delta t) B\left(g+\delta g, g^{+}+\delta g^{+}, 0\right)-\operatorname{Det} \beta(0) B\left(g, g^{+}, 0\right) \\
& =\operatorname{Det} \beta(\delta t) \delta B^{(+)}\left(g, g^{+}, 0\right)+(\operatorname{Det} \beta(\delta t)-1) B\left(g, g^{+}, 0\right)
\end{aligned}
$$

using $\operatorname{Det} \beta(0)=1$. We find that

$$
\begin{aligned}
\operatorname{Det} \beta(\delta t) & =1+\sum_{p} \delta \beta(0)_{p p} \\
& =1-\sum_{p}\left\{L_{p}^{p} \delta t+\sum_{a} K_{p, a}^{p} \delta \tilde{w}_{a}\left(0_{+}\right)\right\}
\end{aligned}
$$

using our previous result (106). Details are given in Appendix 17. Hence we have

$$
\begin{aligned}
\delta B^{\#}\left(g, g^{+}, 0\right)= & {\left[\left(1+\sum_{p}\left(-L_{p}^{p} \delta t-\sum_{a} K_{p, a}^{p} \delta \tilde{w}_{a}\left(0_{+}\right)\right)\right.\right.} \\
& \times\left\{\sum_{p}\left(B\left(g, g^{+}, 0\right) \frac{\overleftarrow{\partial}}{\partial g_{p}}\right) \delta \tilde{g}_{p}(0)+\frac{1}{2} \sum_{p q}\left(B\left(g, g^{+}, 0\right) \frac{\overleftarrow{\partial}}{\partial g_{q}} \frac{\overleftarrow{\partial}}{\partial g_{p}}\right) \delta \tilde{g}_{p}(0) \delta \tilde{g}_{q}(0)\right\} \\
& +\left(\sum_{p}\left(-L_{p}^{p} \delta t-\sum_{a} K_{p, a}^{p} \delta \tilde{w}_{a}\left(0_{+}\right)\right) B\left(g, g^{+}, 0\right)\right]_{\text {stoch aver }}
\end{aligned}
$$

where $\delta \tilde{g}_{p}(0)$ is given by (110) and we now have introduced stochastic variables. Note the $g, g^{+}$are non-stochastic. Carrying out the stochastic averaging using $\overline{\delta \tilde{w}_{a}\left(0_{+}\right)}=0, \overline{\delta \tilde{w}_{a}\left(0_{+}\right) \delta \tilde{w}_{b}\left(0_{+}\right)}=$ $\delta_{a b} \delta t$ and $\overline{\delta \tilde{w}_{a}\left(0_{+}\right) \delta \tilde{w}_{b}\left(0_{+}\right) \delta \tilde{w}_{c}\left(0_{+}\right)}=0$ gives

$$
\begin{aligned}
\delta B^{\#}\left(g, g^{+}, 0\right)= & \sum_{p}\left(B\left(g, g^{+}, 0\right) \frac{\overleftarrow{\partial}}{\partial g_{p}}\right) C^{p}\left(g_{q}\right) \delta t \\
& +\frac{1}{2} \sum_{p q}\left(B\left(g, g^{+}, 0\right) \frac{\overleftarrow{\partial}}{\partial g_{q}} \frac{\overleftarrow{\partial}}{\partial g_{p}}\right)\left(B\left(g_{q}\right) B^{T}\left(g_{q}\right)\right)_{p q} \delta t \\
& -\sum_{p q}\left(B\left(g, g^{+}, 0\right) \frac{\overleftarrow{\partial}}{\partial g_{q}}\right)\left(\sum_{a} K_{p, a}^{p} B_{a}^{q}\left(g_{q}\right)\right) \delta t-B\left(g, g^{+}, 0\right) \sum_{p} L_{p}^{p} \delta t
\end{aligned}
$$

correct to the first order in $\delta t$. The derivation of the first two and the last term in (113) is straight-forward. Details for the other contributions are given in Appendix 17.

The expression (112) for the change in the distribution function based on the Plimak et al [23] ansatz may be compared to that in (108) arising from the Fokker-Planck equation. The expressions are similar, but as we will see they differ in detail. By comparing the terms involving $B\left(g, g^{+}, 0\right)$ and its first and second order Grassmann derivatives, we see that if the 
two expressions are to be the same then certain relationships must be satisfied. For terms involving $B\left(g, g^{+}, 0\right) \frac{\overleftarrow{\partial}}{\partial g_{p}}, B\left(g, g^{+}, 0\right) \frac{\overleftarrow{\partial}}{\partial g_{q}} \frac{\overleftarrow{\partial}}{\partial g_{p}}$ and $B\left(g, g^{+}, 0\right)$ to agree, we require

$$
\begin{aligned}
C^{p}\left(g_{q}\right)-\sum_{q} \sum_{a} K_{q, a}^{q} B_{a}^{p}\left(g_{q}\right) & =+A_{p}+\sum_{q}\left(D_{q p} \frac{\overleftarrow{\partial}}{\partial g_{q}}\right) \\
\left(B\left(g_{q}\right) B^{T}\left(g_{q}\right)\right)_{p q} & =D_{p q} \\
-\sum_{p} L_{p}^{p} & =-\sum_{p}\left(A_{p} \frac{\overleftarrow{\partial}}{\partial g_{p}}\right)+\frac{1}{2} \sum_{p q}\left(D_{p q} \frac{\overleftarrow{\partial}}{\partial g_{q}} \frac{\overleftarrow{\partial}}{\partial g_{p}}\right)
\end{aligned}
$$

The condition (114) is quite different to our previous result (69) that $C^{p}\left(g_{q}\right)=-A_{p}$. However, we find that (see Appendix 17)

$$
\sum_{q} D_{q p} \frac{\overleftarrow{\partial}}{\partial g_{q}}=\sum_{q a} B_{a}^{q} K_{q, a}^{p}-\sum_{q a} K_{q, a}^{q} B_{a}^{p}
$$

so (114) now becomes $C^{p}\left(g_{q}\right)=+A_{p}+\sum_{q a} B_{a}^{q} K_{q, a}^{p}$. Because the $B_{a}^{q}$ and $K_{q, a}^{p}$ arise from the diffusion term this implies that the first term in the Ito stochastic equations would not be wholly determined from the drift term in the Fokker-Planck equation - a feature also present in (83) of Ref [23]. The second condition (115) on the other hand, is exactly the same as in our previous requirement (70). The third condition (116) matches up least well. We find that (see Appendix 17)

$$
\begin{aligned}
\sum_{p q} D_{p q} \frac{\overleftarrow{\partial}}{\partial g_{q}} \frac{\overleftarrow{\partial}}{\partial g_{p}} & =-\sum_{p q a} K_{p, a}^{q} K_{q, a}^{p}+\sum_{p q a} K_{q, a}^{q} K_{p, a}^{p} \\
-\sum_{p}\left(A_{p} \frac{\overleftarrow{\partial}}{\partial g_{p}}\right) & =+\sum_{p} L_{p}^{p}
\end{aligned}
$$

so the two sides of (116) do not agree at all, since (116) now becomes $-\sum_{p} L_{p}^{p}=+\sum_{p} L_{p}^{p}+$ $\frac{1}{2}\left(\sum_{p q a}\left(K_{q, a}^{q} K_{p, a}^{p}-K_{p, a}^{q} K_{q, a}^{p}\right)\right)$. This equation is not valid for the drift vector and diffusion matrix given in (50). Thus with our Ito equations applying, the Plimak et al ansatz does not give the actual change $\delta B\left(g, g^{+}, 0\right)$ in the distribution function over the interval $(0, \delta t)$. Again we conclude that the approach of Plimak et al must be associated with Ito equations for the $\tilde{g}(t)$ that are different to ours.

Thus, we may conclude that our approach to determining the Ito stochastic equations for the stochastic Grassmann variables $\tilde{g}_{p}(t)$ and the Plimak et al [23] ansatz involving stochastic equations for the transformation matrix $\beta$ are not equivalent and cannot be interconverted in such a way that the distribution function at time $\delta t$ would be the same. The approach of Plimak et al must be associated with Ito equations for the $\tilde{g}(t)$ that are different to ours. As Plimak et al do not present the detailed forms for their matrices $m$ and $\delta Q$ we can go no further in discovering what these may be. The two results being inequivalent is not surprising since the basic approaches are different - in our case based on requiring the stochastic average of an arbitrary function of the Grassmann stochastic phase space variables to coincide with the phase space average of the same function using the time dependent distribution function, but in the Plimak et al case based on requiring the distribution function itself to be determined stochastically 
from the initial distribution function with the Grassmann phase space variables being replaced by stochastic phase space variables multiplied by the determinent of the transformation matrix. There is no reason why the results should be the same.

To summarise - by starting from the basic equation (53) we ensure that stochastic expressions such as (59) for the quantum correlation functions or (57) and (58) for Fock state populations and coherences provide the same result as the related phase space average using the distribution function. Furthermore, we obtain explicit expressions (69) and (70) for the classical $C^{p}$ and noise $B_{a}^{p}$ terms in the Ito equations for the stochastic Grassmann phase space variables in terms of the drift $A_{p}$ and diffusion $D_{p q}$ terms in the Fokker-Planck equation, demonstrating by construction (75) how the $B_{a}^{p}$ are obtained. We have also shown how the Ito stochastic equations for the $\tilde{g}(t)$ can be used in numerical calculations of quantum correlation functions etc. We claim that our approach is logical and useful. It is a different approach to that of Plimak et al [23], even though some features (such as using the $B$ distribution) are in common and the final transformations of the stochastic variables $\tilde{g}(t)$ are also linear and involve a c-number stochastic matrix $\beta$. However, as we have seen, our Ito stochastic equations for the $\tilde{g}(t)$ are not equivalent to the Plimak et al stochastic equation for the matrix $\beta$ as they do not lead to the same distribution function at time $\delta t$. As the two approaches are therefore different, it will be of interest to apply both to the same problem and compare the results. This would be especially useful if a problem for which exact solutions could be found.

\section{Summary and Conclusions}

A phase space theory for fermions has been presented for fermion systems based on distribution functions which replace the density operator involving Grassmann phase space variables representing anti-commuting fermion annihilation, creation operators for each of the separate modes. The fermion dstribution function is an even Grassmann function, which we show is unique. Quantum correlation functions, Fock state populations and coherences are given as phase space Grassmann integrals involving even Grassmann distribution functions. Normalised complex $P$ and un-normalised $B$ distribution functions have been treated, though the main focus is on the latter. Fokker-Planck equations for the distribution functions have been obtained, in which the drift vector, diffusion matrix are odd, even Grassmann functions respectively, and explicit expressions for these quantities are given. The diffusion matrix is anti-symmetric, in constrast to being symmetric for bosons. Correspondence rules for the fermion annihilation, creation operators are presented. The proof that Ito stochastic differential equations for stochastic Grassmann variables that replace the phase space variables, which are equivalent to the Fokker-Planck equation can be obtained, with classical and noise contributions constructed from the drift and diffusion terms in Fokker-Planck equation. A total of $2 n^{2}$ Wiener increments are involved, contrasting $2 n$ for the boson case. Quantum correlation functions, populations, coherences are now given by stochastic averages. For the $B$ distribution function case the stochastic Grassmann variables at a later time are related linearly to those at an earlier time via non-Grassmann quantities involving Wiener increments. The stochastic average of products of stochastic Grassmann variables at time $t_{f}$ is given by sums over stochastic averages of products of c-number stochastic quantities, times stochastic averages of products of stochastic Grassmann variables at time $t_{0}$. The stochastic averages of products of stochastic Grassmann variables at time $t_{0}$ can be obtained from the initial conditions. For $B$ distribution function this result shows that numerical calculations are possible - representing Grassmann variables on the computer is not required. The approach to applying the theory in numerical calculations is spelt out in some detail in Section 5.9. A numerical approach based on Eq. (88) is proposed for calculations 
of quantum correlation functions, Fock state populations and coherences, enabling these to be carried out using c-number processes without the need to represent the Grassmann phase space variables on the computer. An analytic application of the separate mode theory to a simple Cooper pair model is presented.

We have compared our theoretical approach to that in Ref [23] showing that our approach involves a different principle for deriving the Ito stochastic equations, resulting in different final Ito equations.

It will be of interest to apply the theory to various topics in degenerate Fermi gases. The utility of the theory could first be tested on some well-understood fermion systems that have been treated by other methods, such determining the size of a Cooper pair or treating Feshbach resonance in Fermi gases or deriving the two fluid hydrodynamuic equations for Fermi liquids. Some further development of the formalism could be worthwhile, such as obtaining formulae for two-time quantum correlation functions, as these are linked to fermionic excitations. Some work on this already exists [28]. Degenerate quantum gases involving both fermions and bosons often occur, so expanding the formalism to include both is desirable, and again some work has already been done [24], [39]. Also, extending the phase space method to include stochastic gauges could be worthwhile to facilitate numerical calculations, and here some work on this for both bosons [50] and fermions [29] using c-number phase space theory has been carried out. It is clearly desirable to carry out numerical applications on fermion systems to fully test out the Grassmann phase space theory.

\section{Acknowledgements}

This work was supported by the Australian Research Council Centre of Excellence for Quantum Atom Optics, the Royal Society and the Wolfson foundation. The authors thank J. Corney, P. Drummond, M. Olsen, B. Opanchuk and L. Plimak for helpful discussions. The authors are grateful to Dr C Gilson, Dept. of Applied Maths., University of Glasgow for the first derivation of a solution to the factorisation $D=B B^{T}$ for anti-symmetric Grassmann matrices $D$ and to a referee for directing our attention to issues involved in numerical calculations. 


\section{References}

[1] N. H. March, W. H. Young and S. Sampanthar, The Many-Body Problem in Quantum Mechanics, (Cambridge University Press, Cambridge, UK, 1967).

[2] P. A. M. Dirac, Proc. Cambridge Philos. Soc. 26, 376 (1930).

[3] J. Frenkel, Wave Mechanics (Oxford University Press, Oxford, UK, 1934).

[4] R. P. Feynman and A. R. Hibbs, Quantum Mechanics and Path Integrals, (McGraw-Hill, New York, USA, 1965).

[5] A. Montina and Y. Castin: Phys. Rev. A 73, 013618 (2006).

[6] S. S. Schweber, Introduction to Relativistic Quantum Field Theory, (Harper and Row, New York, USA, 1961).

[7] H. Risken, The Fokker-Planck Equation, 2nd Edition (Springer-Verlag, Berlin, Germany, 1989).

[8] R. J. Glauber, Phys. Rev. 131, 2766 (1963).

[9] E. C. G. Sudarshan. Phys. Rev. Lett. 10, 277 (1963).

[10] K. Husimi, Proc. Phys. Math. Soc. Jpn. 22, 264 (1940).

[11] E. P. Wigner, Phys. Rev. 40, 749 (1930).

[12] J. E. Moyal, Proc. Cambridge Philos. Soc. 45, 99 (1948), ibid 45, 545 (1949).

[13] C. W. Gardiner and P. Zoller, Quantum Noise, 3rd Edition (Springer-Verlag, Berlin, Germany, 2004).

[14] S. M. Barnett and P. M. Radmore, Methods in Theoretical Quantum Optics, (Clarendon Press, Oxford, UK, 1997).

[15] C. C. Gerry and P. L. Knight, Introductory Quantum Optics, (Cambridge University Press, Cambridge, UK, 2005).

[16] P. D. Drummond and C. W. Gardiner, J. Phys. A: Math. Gen. 13, 2353 (1980).

[17] D. F. Walls and G. J. Milburn, Quantum Optics, (SpringerVerlag, Berlin, Germany, 1994).

[18] J. F. Corney and P. D. Drummond, J. Phys. A: Math. Gen. 39, 269 (2006).

[19] J. F. Corney and P. D. Drummond, Phys. Rev. A 68, 063822 (2003).

[20] F. A. Berezin, The Method of Second Quantization, (Academic Press, New York, USA, 1966).

[21] J. Zinn-Justin, Quantum Field Theory and Critical Phenomena, (Clarendon Press, Oxford, UK, 2002).

[22] K. E. Cahill and R. J. Glauber, Phys. Rev. A 59, 1538 (1999).

[23] L. Plimak, M. J. Collett and M. K. Olsen, Phys. Rev. A 64, 063409 (2001). 
[24] B. J. Dalton, B. M. Garraway, J. Jeffers and S. M. Barnett,. Annals of Physics, 334, 100 (2013).

[25] V. V. Shcherbakov, Theor. Math. Phys. 96, 1323 (1993).

[26] C. Anastopoulos and B. L. Hu, Phys. Rev. A 62, 033801 (2000).

[27] S. Shresta, C. Anastopoulos, A. Dragulescu and B. L. Hu, Phys. Rev. A 71, 022109 (2005).

[28] L. Plimak and S. Stenholm, Annals of Physics, 324, 600 (2009).

[29] J. F. Corney and P. D. Drummond, Phys. Rev. B 73, 125112 (2006).

[30] A. Gatti, H. Wiedemann, L.Lugiato, I. Marzoli, G-L. Oppo and S. M. Barnett, Phys. Rev. A 56, 877 (1997).

[31] M.J. Steel, M.K. Olsen, L.I. Plimak, P.D. Drummond, S.M. Tan, M.J. Collett, D.F. Walls, R. Graham, Phys. Rev. A 58, 4824 (1998).

[32] P. Deuar and P. D. Drummond, Phys. Rev. A 66, 033812 (2002).

[33] B. J. Dalton, J. Phys. C: Conf. Ser. 67 , 012059 (2007).

[34] S. E. Hoffmann, J. F. Corney and P. D. Drummond, Phys. Rev. A 78, 013622 (2008).

[35] B. J. Dalton. Annals of Physics, 326, 668 (2011).

[36] B. J. Dalton. Annals of Physics, 327, 2432 (2012).

[37] R. Graham and H. Haken, Z Phys 235, 166 (1970).

[38] B. J. Dalton, J. Jeffers and S. M. Barnett,.Grassmann Phase Space Theory for Fermions. II. Field Theory. In preparation. (2016).

[39] B. J. Dalton, J. Jeffers and S. M. Barnett,.Phase Space Methods for Degenerate Quantum Gases (Oxford University Press, Oxford, UK, 2015).

[40] G. C. Wick, A. S. Wightman and E. P. Wigner, Phys. Rev. 88, 101 (1952).

[41] B. J. Dalton, L. Heaney, J. Goold, B. M. Garraway and Th. Busch, New. J. Phys. 16, 013026 (2014).

[42] A. J. Leggett, Rev. Mod. Phys. 73, 307 (2001).

[43] R. Bach and K. Rzazewskii, Phys. Rev. A 70, 063622 (2004).

[44] B. J. Dalton and S. Ghanbari, J. Mod. Opt. 59, 287 (2012), ibid 60, 602 (2013).

[45] M. Kaku, Quantum Field Theory (Oxford University Press, Oxford, UK, 1993), p52.

[46] T. Matsubara, Prog. Theor. Phys. 14 , 351 (1955).

[47] C. W. Gardiner, Handbook of Stochastic Methods for Physics, Chemistry and the Natural Sciences (Springer-Verlag, Berlin, Germany, 1983).

[48] T. Takagi, Japan J. Math. 1, 83 (1925). 
[49] R. A. Horn and C. R. Johnson, Matrix Analysis, (Cambridge University Press, Cambridge, UK, 1985).

[50] P. Deuar and P. D. Drummond, J. Phys. A 39, 269 (2006).

[51] T. Tyc, B. Hamilton, B. C. Sanders and W. D. Oliver, Fdns. Phys. 37, 1519 (2007). 


\section{Appendix A - Grassmann Algebra and Calculus}

\subsection{Algebra}

Grassmann variables are mathematical quantities that satisfy all the rules of algebra applying for complex numbers, except for the rules for multiplication and complex conjugation. A full account of the properties of Grassmann variables is given in [20] (see also [24]).

In the case of multiplication anti-commutation rules apply both for pairs of Grassmann variables $h_{i}, h_{j}$ and for Grassmann variables and fermion mode annihilation, creation operators.

$$
\left\{h_{i}, h_{j}\right\}=0 \quad\left\{h_{i}, \hat{c}_{j}\right\}=\left\{h_{i}, \hat{c}_{j}^{\dagger}\right\}=0
$$

It follows then that the square and higher powers of Grassmann variables are zero $h_{i}^{2}=h_{i}^{3}=. .=$ 0 . As a consequence it is straight-forward to show that no inverse exists for Grassmann variables. However, Grassmann variables commute with c-numbers and boson mode annihilation, creation operators and with the vacuum state $|0\rangle$. It then follows that

$$
h_{i}\left|\nu_{1}, \cdots, \nu_{n}\right\rangle=(-1)^{\eta}\left|\nu_{1}, \cdots, \nu_{n}\right\rangle h_{i}
$$

where $\eta=\sum_{i} \nu_{i}$ is the total occupancy, showing that Grassmann numbers commute or anticommute with Fock states (4) depending on whether the number of fermions is even or odd.

The rules for complex conjugation of products of Grassmann variables and for products of Grassmann variables and fermion mode annihilation, creation operators are

$$
\left(h_{i} h_{j}\right)^{*}=h_{j}^{*} h_{i}^{*} \quad\left(h_{i} \hat{c}_{j}\right)^{\dagger}=\hat{c}_{j}^{\dagger} h_{i}^{*} \quad\left(h_{i} \hat{c}_{j}^{\dagger}\right)^{\dagger}=\hat{c}_{j} h_{i}^{*}
$$

These are similar to the rules for the Hermitian adjoints of operator products.

\subsection{Grassmann Functions}

A general Grassmann function of Grassmann variables $h_{1}, h_{2}, \cdots h_{n}$ has the following form

$$
\begin{aligned}
f\left(h_{1}, h_{2}, \cdots, h_{n}\right)= & f_{0}+\sum_{i} f_{i} h_{i}+\sum_{i<j} f_{i j} h_{i} h_{j}+\sum_{i<j<k} f_{i j k} h_{i} h_{j} h_{k}+\cdots \\
& +f_{123 . . n} h_{1} h_{2} . . h_{n}
\end{aligned}
$$

where $f_{0}, f_{i}, f_{i j}, \cdots, f_{12 . . n}$ are c-numbers. In any term an $h_{i}$ never appears twice, since by applying the anti-commutation rule repeated variables could be placed together to give zero.

The dependence of a Grassmann function dependence on any particular variable $h_{i}$ is linear

$$
\begin{aligned}
& f\left(h_{1}, h_{2}, \cdots, h_{n}\right) \\
= & a_{i}\left(h_{1}, \cdots, h_{i-1}, h_{i+1}, \cdots h_{n}\right)+b_{i}^{L}\left(h_{1}, \cdots, h_{i-1}, h_{i+1}, \cdots h_{n}\right) h_{i} \\
= & a_{i}\left(h_{1}, \cdots, h_{i-1}, h_{i+1}, \cdots h_{n}\right)+h_{i} b_{i}^{R}\left(h_{1}, \cdots, h_{i-1}, h_{i+1}, \cdots h_{n}\right)
\end{aligned}
$$

where $a_{i}\left(h_{1}, \cdots, h_{i-1}, h_{i+1}, \cdots h_{n}\right)$ and $b_{i}^{L, R}\left(h_{1}, \cdots, h_{i-1}, h_{i+1}, \cdots h_{n}\right)$ are independent of $h_{i}$, and in general two alternative forms are possible depending on whether $h_{i}$ is place on the left or right side in the second term.

New Grassmann functions can be defined as functions of an original Grassmann function $f\left(h_{1}, \cdots, h_{i}, \cdots, h_{n}\right)$. Important examples include powers and exponentials which are defined in the same way as for c-numbers:

$$
\begin{aligned}
f(h)^{n} & \equiv f f \cdots f \quad n \text { times } \\
\exp [f(h)] & =1+f+f^{2} / 2 !+f^{3} / 3 !+\cdots .
\end{aligned}
$$


Given that the original Grassmann function terminates after the $n$th order, it is not surprising that even if a function is defined by an infinite series, the series will often terminate. A simple example is $\exp (h)=1+h$, as all the higher order terms are zero.

Grassmann functions are even (odd) if the Grassmann function only contains terms with even (odd) numbers of variables $f_{i}=f_{i j k}=. .=0\left(f_{0}=f_{i j}=. .=0\right)$. Note that in Eq.(123) for even Grassmann functions $a_{i}$ is even and $b_{i}^{L}=-b_{i}^{R}$, whereas for odd functions $a_{i}$ is odd and $b_{i}^{L}=+b_{i}^{R}$. By grouping the terms in Eq.(122) any Grassmann function can be expressed as the sum of and even and an odd Grassmann function:

$$
f=f_{E}+f_{O} .
$$

For an arbitary pair of functions $f, h$ as in (125) we can then show that the commuatator is

$$
[f, g]=2 f_{O} h_{O}
$$

indicating that a even Grassmann function will commute with any other Grassmann fuction but odd Grassmann function do not commute. In fact, they anti-commute

$$
\begin{aligned}
\left\{f_{O}, h_{O}\right\} & =f_{O} h_{O}+h_{O} f_{O}=0 \\
f_{O}^{2} & =f_{O}^{3}=\cdots=0
\end{aligned}
$$

and clearly, the square and any higher power of any odd Grassmann function is zero. As $2 f_{O} h_{O}$.is even, it follows that the commutator of $[f, h]$ with either $f$ or $h$, or indeed any other Grassmann function, will be zero

$$
[f,[f, h]]=[h,[f, h]]=[g,[f, h]]=0 .
$$

Although Grassmann variables have no inverse, Grassmann functions with a non-zero constant term $f_{0}$ are invertible. Any Grassmann function with $f_{0}=1$ can be written as $\left(1+h_{1} f\left(h_{2}, \cdots, h_{n}\right)\right)$, where $f\left(h_{2}, \cdots, h_{n}\right)$ is a Grassmann function of the other g-numbers. As

$$
\begin{aligned}
& \left(1+h_{1} f\left(h_{2}, \cdots, h_{n}\right)\right)\left(1-h_{1} f\left(h_{2}, \cdots, h_{n}\right)\right)=1 \\
& \quad=\left(1-h_{1} f\left(h_{2}, \cdots, h_{n}\right)\right)\left(1+h_{1} f\left(h_{2}, \cdots, h_{n}\right)\right) .
\end{aligned}
$$

Thus the left and right inverse of $\left(1+h_{1} f\left(h_{2}, \cdots, h_{n}\right)\right)$ is $\left(1-h_{1} f\left(h_{2}, \cdots, h_{n}\right)\right)$. More generally, the inverse of a function with $f_{0} \neq 0$ can be found by multiplying appropriately by $f_{0}$.

The Baker-Hausdorff theorem applies to Grassmann functions:

$$
\exp (f) \times \exp (h)=\exp (f+h) \times \exp \left(\frac{1}{2}[f, h]\right) .
$$

If either $f$ or $g$ (or both) is even then the normal c-number rule $\exp (f) \times \exp (h)=\exp (f+h)$ applies.

The exponential of a Grassmann function has an inverse given as the exponential of minus the Grassmann function

$$
\exp (f) \times \exp (-f)=\exp (-f) \times \exp (f)=1
$$

as can readily be obtained from (130). The exponential transformation law for general noncommuting operators has the general form

$$
\exp (A) B \exp (-A)=B+[A, B]+\frac{1}{2 !}[A,[A, B]]+\frac{1}{3 !}[A,[A,[A, B]]]+\cdots .
$$


and as Grassmann functions have the same algebraic properties, it then follows that for a pair a Grassmann functions

$$
\exp (f) \times h \times \exp (-f)=g+[f, h] .
$$

The series in (132) terminates after the second term because $[f,[f, h]]=0$.

The complex conjugate of the product of two Grassmann functions is the product of the two conjugates in reverse order

$$
\left(f_{1}(g) f_{2}(g)\right)^{*}=f_{2}(g)^{*} f_{1}(g)^{*} .
$$

This follows from the rules for complex conjugation of Grassmann numbers.

Finally, in manipulating products of Grassmann variables there are several useful rules available for: (a) re-ordering the terms in the opposite order (see (135)), (b) separating products of pairs of different types of g-numbers into the product of terms of the same type (see (136)) and (c) transfering products of two different types of g-numbers into the opposite order (see (137)).

(a) Reordering

$$
h_{1} h_{2} \cdots h_{n}=h_{n} \cdots h_{2} h_{1}(-1)^{n(n-1) / 2}
$$

(b) Separating

$$
g_{1} h_{1} g_{2} h_{2} \cdots g_{n} h_{n}=g_{1} g_{2} \cdots g_{n} h_{1} h_{2} \cdots h_{n}(-1)^{n(n-1) / 2}
$$

(c) Transfering

$$
h_{1} h_{2} \cdots h_{n} g_{1} g_{2} \cdots g_{n}=g_{1} g_{2} \cdots g_{n} h_{1} h_{2} \cdots h_{n}(-1)^{n^{2}}
$$

\subsection{Calculus}

Unlike the calculus involving c-number functions, Grassmann calculus is not based on defining differentiation and integration via limiting processes involving small changes of the variable being differentiated or integrated, Instead, Grassmann differentiation and integration are introduced in a formal way, based on formal rules for differentiating or integrating the fundamental quantities 1 and $h_{i}$. Unlike c-number calculus both left and right differentiation and integration processes are defined. These rules are then extended to apply to more general Grassmann functions via writing the Grassmann function in one of the two forms in Eq.(123), and then applying the differentiation or integration process to each of the two terms in an obvious way. To consider integration we need to introduce the concept of Grassmann differentials $d h_{1}, d h_{2}, \cdots, d h_{n}$ which satisfy all the standard rules for g-numbers:

$$
\begin{aligned}
d h_{i} d h_{j}+d h_{j} d h_{i} & =0 & & (i, j=1, \cdots, n) \\
h_{i} d h_{j}+d h_{j} h_{i} & =0 & & (i, j=1, \cdots, n)
\end{aligned}
$$

and which also anti-commute with fermion annihilation, creation operators, and commute with c-numbers and boson operators.

Left and right differentiation of the Grassmann function $h_{j}$ with respect to $h_{i}$ is defined by

$$
\frac{\vec{\partial}}{\partial h_{i}} h_{j}=h_{j} \frac{\overleftarrow{\partial}}{\partial h_{i}}=\delta_{i j}
$$

Left and right integration of the Grassmann function $h_{j}$ or 1 with respect to $h_{i}$ is defined by 


$$
\int d h_{i} 1=0 \quad \int d h_{i} h_{j}=\delta_{i j} \quad \int 1 d h_{i}=0 \quad \int h_{j} d h_{i}=-\delta_{i j}
$$

For general Grassmann functions, we proceed by moving the Grassmann variable being differentiated or integrated either to the left or right of all other Grassmann variables (depending on whether left or right differentiation or integration applies) and then apply the above rules. For example, the left differentiation and integration of the function in Eq.(123) gives

$$
\frac{\vec{\partial}}{\partial h_{i}} f\left(h_{1}, \cdots, h_{n}\right)=b_{i}^{R}\left(h_{1}, \cdots, h_{i-1}, h_{i+1}, \cdots h_{n}\right)=\int d h_{i} f\left(h_{1}, \cdots, h_{n}\right)
$$

showing that left differentiation and integration give the same result - a feature quite different to that in c-number calculus. For right differentiation and intgration there is a sign difference

$$
f\left(h_{1}, \cdots, h_{n}\right) \frac{\overleftarrow{\partial}}{\partial h_{i}}=b_{i}^{L}\left(h_{1}, \cdots, h_{i-1}, h_{i+1}, \cdots h_{n}\right)=-\int f\left(h_{1}, \cdots, h_{n}\right) d h_{i}
$$

It then follows that for even and odd Grassmann functions there are simple relations between the left and right derivatives

$$
\frac{\vec{\partial}}{\partial h_{i}} f_{E}\left(h_{1}, \cdots, h_{n}\right)=-f_{E}\left(h_{1}, \cdots, h_{n}\right) \frac{\overleftarrow{\partial}}{\partial h_{i}} \quad \frac{\vec{\partial}}{\partial h_{i}} f_{O}\left(h_{1}, \cdots, h_{n}\right)=+f_{O}\left(h_{1}, \cdots, h_{n}\right) \frac{\overleftarrow{\partial}}{\partial h_{i}}
$$

Multiple differentiation and integration processes are defined by applying the single processes in succession. Thus

$$
\begin{aligned}
\frac{\vec{\partial}}{\partial h_{n}} \cdots \frac{\vec{\partial}}{\partial h_{2}} \frac{\vec{\partial}}{\partial h_{1}} f(h)=\frac{\vec{\partial}}{\partial h_{n}}\left(\cdots\left(\frac{\vec{\partial}}{\partial h_{2}}\left(\frac{\vec{\partial}}{\partial h_{1}} f(h)\right)\right)\right) \\
f(h) \frac{\overleftarrow{\partial}}{\partial h_{1}} \frac{\overleftarrow{\partial}}{\partial h_{2}} \cdots \frac{\overleftarrow{\partial}}{\partial h_{n}}=\left(\left(\left(f(h) \frac{\overleftarrow{\partial}}{\partial h_{1}}\right) \frac{\overleftarrow{\partial}}{\partial h_{2}}\right) \cdots \cdots \frac{\overleftarrow{\partial}}{\partial h_{n}}\right)
\end{aligned}
$$

with analogous results for mixed derivatives where both left and right differentation processes occur.

In the case of multiple left integration

$$
\int \cdots \int d h_{s} \cdots d h_{j} d h_{i} f(h) \equiv \int d h_{s}\left(\cdots\left(\int d h_{j}\left(\int d h_{i} f(h)\right)\right) \cdots\right) .
$$

with an analogous result for right integration or for mixed integration. When a Grassmann function is differentiated or integrated more than once with respect to the same Grassmann variable, the result will be zero since the first process leads to a Grassmann function that is no longer dependent on that variable.

The left derivative of a Grassmann function as in Eq.(122) over all the Grassmann variables $h_{1}, \cdots, h_{n}$ is given by

$$
\frac{\vec{\partial}}{\partial h_{n}} \cdots \frac{\vec{\partial}}{\partial h_{2}} \frac{\vec{\partial}}{\partial h_{1}} f(h)=f_{123 . . n}
$$

which is a c-number. The left integral of the same Grassmann function over all the Grassmann variables $h_{1}, \cdots, h_{n}$ is given by

$$
\begin{aligned}
\int d \mathbf{h} f(h) & \equiv \int \cdots \int d h_{n} \cdots d h_{i} \cdots d h_{2} d h_{1} f\left(h_{1}, \cdots, h_{n}\right) \\
& =f_{123 . . n}
\end{aligned}
$$


which is the same. When writing $d \mathbf{h}$, of course, we mean that the product of Grassmann differentials is in a particular order: $d \mathbf{h}=d h_{n} \cdots d h_{i} \cdots d h_{2} d h_{1}=\prod_{i} d h_{i}$. An important result is that the full Grassmann integral of the derivative of a Grassmann function is zero:

$$
\begin{aligned}
\int d \mathbf{h} \frac{\vec{\partial}}{\partial h_{i}} f(h) & =0 \\
\int d \mathbf{h} f(h) \frac{\overleftarrow{\partial}}{\partial h_{i}} & =0
\end{aligned}
$$

This is because differentiation removes the $g_{i}$ variable and so the fundamental rules require that the integral over $d h_{i}$ must give zero. This is in contrast to the rule for normal calculus which would give the function itself, evaluated on the boundaries if the integral is definite. Whether the final result is zero then depends on whether the function becomes zero on the boundary.

\subsection{Formal Rules - Differentiation}

As in c-number calculus product rules for differentiation, integration by parts rules etc apply also in the case of Grassmann calculus. There are however important differences in the form of these rules to those for c-number calculus. This can be traced back to the anti-commutation features of Grassmann variables as well as to the different definitions for differentiation and integration.

Carrying out two right or left derivatives in opposite order gives results that differ by a minus sign. We note that any Grassmann function can be written in the form

$$
f(h)=F_{0}\left(\overline{h_{1,2}}\right)+h_{1} F_{1}\left(\overline{h_{1,2}}\right)+h_{2} F_{2}\left(\overline{h_{1,2}}\right)+h_{1} h_{2} F_{1,2}\left(\overline{h_{1,2}}\right)
$$

where the $F\left(\overline{h_{1,2}}\right)$ are functions of all the Grassmann variables except $h_{1}$ and $h_{2}$. For left diferentiation we have

$$
\frac{\vec{\partial}}{\partial h_{1}} \frac{\vec{\partial}}{\partial h_{2}} f(h)=F_{1,2}\left(\overline{h_{1,2}}\right)=-\frac{\vec{\partial}}{\partial h_{2}} \frac{\vec{\partial}}{\partial h_{1}} f(h) .
$$

An analogous result applies for right differentiation. The mixed left and right differentiation can be applied in either order. To see this we note that any Grassmann function can also be written in the form

$$
f(h)=A_{0}\left(\overline{h_{1,2}}\right)+h_{1} A_{1}\left(\overline{h_{1,2}}\right)+A_{2}\left(\overline{h_{1,2}}\right) g_{2}+h_{1} A_{1,2}\left(\overline{h_{1,2}}\right) h_{2}
$$

where the $A\left(\overline{h_{1,2}}\right)$ are functions of all the Grassmann variables except $h_{1}$ and $h_{2}$. It then follows that

$$
\left(\frac{\vec{\partial}}{\partial h_{1}} f(h)\right) \frac{\overleftarrow{\partial}}{\partial h_{2}}=A_{1,2}\left(\overline{h_{1,2}}\right)=\frac{\vec{\partial}}{\partial h_{1}}\left(f(h) \frac{\overleftarrow{\partial}}{\partial h_{2}}\right)=\frac{\vec{\partial}}{\partial h_{1}} f(h) \frac{\overleftarrow{\partial}}{\partial h_{2}}
$$

It may also be shown that

$$
\frac{\vec{\partial}}{\partial h_{1}} f(h) \frac{\overleftarrow{\partial}}{\partial h_{2}}=-\frac{\vec{\partial}}{\partial h_{2}} f(h) \frac{\overleftarrow{\partial}}{\partial h_{1}}
$$

for both even and odd Grassmann functions, showing that mixed left and right differentiation can also be swapped subject to a sign change. 
Product rules for differentiation can be derived. These depend on whether the factors are even or odd Grassmann functions:

$$
\begin{aligned}
& \frac{\vec{\partial}}{\partial h_{i}}\left(f_{1}^{E} f_{2}\right)=\left(\frac{\vec{\partial}}{\partial h_{i}} f_{1}^{E}\right) f_{2}+f_{1}^{E}\left(\frac{\vec{\partial}}{\partial h_{i}} f_{2}\right) \\
& \frac{\vec{\partial}}{\partial h_{i}}\left(f_{1}^{O} f_{2}\right)=\left(\frac{\vec{\partial}}{\partial h_{i}} f_{1}^{O}\right) f_{2}-f_{1}^{O}\left(\frac{\vec{\partial}}{\partial h_{i}} f_{2}\right) \\
& \left(f_{2} f_{1}^{E}\right) \frac{\overleftarrow{\partial}}{\partial h_{i}}=f_{2}\left(f_{1}^{E} \frac{\overleftarrow{\partial}}{\partial h_{i}}\right)+\left(f_{2} \frac{\overleftarrow{\partial}}{\partial h_{i}}\right) f_{1}^{E} \\
& \left(f_{2} f_{1}^{O}\right) \frac{\overleftarrow{\partial}}{\partial h_{i}}=f_{2}\left(f_{1}^{O} \frac{\overleftarrow{\partial}}{\partial h_{i}}\right)-\left(f_{2} \frac{\overleftarrow{\partial}}{\partial h_{i}}\right) f_{1}^{O}
\end{aligned}
$$

\subsection{Taylor Series}

The Grassmann numbers, unlike c-numbers, do not have a property of size or magnitude. To see this we need only note that the square of any Grassmann number is zero. It is, nevertheless, useful to introduce the counterpart of a variation of a Grassmann function by replacing each g-number, $h_{k}$, by $h_{k}+\delta h_{k}$, where $\delta h_{k}$ is a further Grassmann variable. This allows us to express a Grassmann function such as in Eq.(122) in the form of a Taylor series, with the coefficients of the powers of the $\delta h_{k}$ being given by Grassmann derivatives. These are Grassmann functions of the $h_{i}$. The Taylor series correct to second order is given by

$$
\begin{aligned}
& f(h+\delta h)-f(h) \\
= & \sum_{k} \delta h_{k}\left\{\frac{\vec{\partial}}{\partial h_{k}} f(h)\right\}+\frac{1}{2} \sum_{k l} \delta h_{k} \delta h_{l}\left\{\frac{\vec{\partial}}{\partial h_{l}} \frac{\vec{\partial}}{\partial h_{k}} f(h)\right\} \\
= & \sum_{k}\left\{f(h) \frac{\overleftarrow{\partial}}{\partial h_{k}}\right\} \delta h_{k}+\frac{1}{2} \sum_{k l}\left\{f(h) \frac{\overleftarrow{\partial}}{\partial h_{k}} \frac{\overleftarrow{\partial}}{\partial h_{l}}\right\} \delta h_{l} \delta h_{k}
\end{aligned}
$$

giving the left and right derivative forms, where there are no order restrictions on the double sums. Note in the second order terms the processes occur in the order kllk.

\subsection{Useful Rules - Integration}

We now establish a useful rule for how Grassmann multiple integrals change under linear transformations of the Grassmann variables. The original $g_{i}$ are replaced by new $h_{j}$ via a linear transformation with c-number coefficients as in (157). The differentials $d g_{i}$ are assumed to transform as in (158), where we cannot just assume that the differential transforms in the same way as the g-number. For non-singular c-number matrices $A, B$

$$
\begin{aligned}
g_{i} & =\sum_{k} A_{i k} h_{k} \\
d g_{i} & =\sum_{k} B_{i k} d h_{k}
\end{aligned}
$$

It is straightforward to show that $h_{k} h_{l}+h_{l} h_{k}=0, h_{k} d h_{l}+d h_{l} h_{k}=0$ and $d h_{k} d h_{l}+d h_{l} d h_{k}=0$ for any $A, B$, so the new quantities $h_{k}$ and $d h_{l}$ satisfy the same anti-commutation rules as the 
old. The matrices $A, B$ however are not independent because the Grassmann integrals must yield the standard results

$$
\int d h_{k} 1=0 \quad \int d h_{k} h_{l}=\delta_{k l}
$$

The first result just follows from Eq.(158). From the second we can easily show that

$$
\begin{aligned}
B & =\left(A^{T}\right)^{-1}=\left(A^{-1}\right)^{T} \\
d g_{i} & =\sum_{k}\left(A^{-1}\right)_{k i} d h_{k}
\end{aligned}
$$

so that the transformation rule for the differentials involves the transpose of the inverse of the matrix for transforming the g-numbers. For the c-number case we would have found $B=A$.

We can now use these results to transform of the product of all g-numbers and of all differentials

$$
\begin{aligned}
g_{1} \cdots g_{n} & =\sum_{k_{1} \cdots k_{n}} A_{1 k_{1}} h_{k_{1}} \cdots A_{1 k_{n}} h_{k_{n}} \\
& =\sum_{k_{1} \cdots k_{n}} A_{1 k_{1}} \cdots A_{1 k_{n}} \epsilon\left(k_{1}, \cdots, k_{n}\right) h_{1} \cdots h_{i} \cdots h_{n}
\end{aligned}
$$

where $\epsilon\left(k_{1}, \cdots, k_{n}\right)=+1,-1$ if $k_{1}, \cdots, k_{n}$ is an even, odd permutation $P\left(1 \rightarrow k_{1} ; 2 \rightarrow\right.$ $\left.k_{2} ; \cdots ; n \rightarrow k_{n}\right)$ of the numbers $1,2, \cdots, n$ and $\epsilon\left(k_{1}, \cdots, k_{n}\right)=0$ if $k_{1}, \cdots, k_{n}$ is not a permutation (and thus contains two $k_{i}$ that are the same). This result follows because if any pair of $h_{k_{i}}$ are the same, then the right side is zero and if the $h_{k_{i}}$ are all different, then we can rearrange the order to be $h_{1} h_{2} \cdots h_{n}$ multiplied by the factor $\epsilon\left(k_{1}, \cdots, k_{n}\right)$. As the multiple summation generates the determinant of the matrix $A$ this shows the following important transform results:

$$
\begin{aligned}
g_{1} \cdots g_{n} & =(\operatorname{Det} A) h_{1} h_{2} \cdots h_{i} \cdots h_{n} \\
d g_{n} \cdots d g_{i} \cdots d g_{2} d g_{1} & =(\operatorname{Det} A)^{-1} d h_{n} \cdots d h_{i} \cdots d h_{2} d h_{1} .
\end{aligned}
$$

the result for the differentials following (163) by just replacing $B$ by $\left(A^{-1}\right)^{T}$.

Consider the function $e(h) \equiv e\left(h_{1}, \cdots, h_{n}\right)$ obtained by substituting for each $g_{i}$ in the function $f(g) \equiv f\left(g_{1}, \cdots, g_{n}\right)$, thus $e(h)=f(g(h))$. We see that the transformation rule for the Grassmann integral is

$$
\int d g_{1} d g_{2} \cdots d g_{n} f(g)=(\operatorname{Det} \mathrm{A})^{-1} \int d h_{1} d h_{2} \cdots d h_{n} e(h)
$$

This differs from the usual c-number result involving the Jacobian, in which the multiplying factor would have been Det A rather than (Det A $)^{-1}$. The order of the differentials on each side can be reversed.

A useful result involves the double integral with $d^{2} h_{i}=d h_{i}^{*} d h_{i}$ of the exponential function $\exp \left(-h_{j}^{*} h_{k}\right)$

$$
\begin{aligned}
\int d^{2} h_{i} \exp \left(-h_{j}^{*} h_{k}\right) & =\int d^{*} h_{i} d h_{i}\left(1-h_{j}^{*} h_{k}\right) \\
& =-\int d^{*} h_{i} d h_{i} h_{j}^{*} h_{k} \\
& =+\int d^{*} h_{i} h_{j}^{*} \int d h_{i} h_{k} \\
& =\delta_{i j} \delta_{i k} .
\end{aligned}
$$

An analogous result applies to $\int d^{2} h_{i} \exp \left(-h_{j}^{+} h_{k}\right)$ with $d^{2} h_{i}=d h_{i}^{+} d h_{i}$, since we often employ two Grassmann variables $h_{i}, h_{i}^{+}$for each quantum mode, which are not related via complex conjugation. 
Another useful example involves the integral of a Grassmann function $F\left(h, h^{+}\right)$of two Grassmann variables $h, h^{+}$:

$$
\int d h^{+} d h F\left(h, h^{+}\right)(h-g)\left(h^{+}-g^{+}\right)=F\left(g, g^{+}\right) .
$$

Note the order of the factors.

A further useful example involves an analogy to the Fourier theorem

$$
\int d k^{+} d k F\left(k, k^{+}\right) \int d g^{+} d g \exp \left(i g\left[h^{+}-k^{+}\right]\right) \exp \left(i[h-k] g^{+}\right)=F\left(h, h^{+}\right)
$$

The previous two results can be generalised to cases where $F\left(h, h^{+}\right)$also depends on further Grassmann variables.

The full Grassmann integral of a Grassmann derivative of a Grassmann function $f\left(h, h^{+}\right)$is zero:

$$
\begin{aligned}
\int d^{2} \mathbf{h} \frac{\vec{\partial}}{\partial h_{i}} f\left(h, h^{+}\right) & =\int d^{2} \mathbf{h} \frac{\vec{\partial}}{\partial h_{i}^{+}} f\left(h, h^{+}\right)=0 \\
\int d^{2} \mathbf{h} f\left(h, h^{+}\right) \frac{\overleftarrow{\partial}}{\partial h_{i}} & =\int d^{2} \mathbf{h} f\left(h, h^{+}\right) \frac{\overleftarrow{\partial}}{\partial h_{i}^{+}}=0 .
\end{aligned}
$$

where here $d^{2} h_{i}=d h_{i}^{+} d h_{i}$. As before, this is because the differentiation from either left or right removes the $h_{i}$ or $h_{i}^{+}$variable from $f$, so the integral over each of these variables gives zero. A similar result applies when each mode is associated with a complex pair Grassmann variables $h_{i}$ and $h_{i}^{*}$ and a Grassmann function $f\left(h, h^{*}\right)$ is differentiated with respect to $h_{i}$ or $h_{i}^{*}$. In this case $d^{2} h_{i}=d h_{i}^{*} d h_{i}$.

Formulae for integration by parts results can be derived from the last equation by considering the product of two functions $e\left(g, g^{+}\right) f\left(g, g^{+}\right)$and applying the product differentiation rules. We find that, with $d^{2} g_{i}=d g_{i}^{+} d g_{i}$,

$$
\begin{aligned}
\int d^{2} \mathbf{h}\left(\frac{\vec{\partial}}{\partial h_{i}} e\left(h, h^{+}\right)\right) f\left(h, h^{+}\right) & =-\sigma(e) \int d^{2} \mathbf{h} e\left(h, h^{+}\right)\left(\frac{\vec{\partial}}{\partial h_{i}} f\left(h, h^{+}\right)\right) \\
\int d^{2} \mathbf{h}\left(f\left(h, h^{+}\right) \frac{\overleftarrow{\partial}}{\partial h_{i}}\right) e\left(h, h^{+}\right) & =-\sigma(e) \int d^{2} \mathbf{h} f\left(h, h^{+}\right)\left(e\left(h, h^{+}\right) \frac{\overleftarrow{\partial}}{\partial h_{i}}\right) \\
\int d^{2} \mathbf{h}\left(\frac{\vec{\partial}}{\partial h_{i}^{+}} e\left(h, h^{+}\right)\right) f\left(h, h^{+}\right) & =-\sigma(e) \int d^{2} \mathbf{h} e\left(h, h^{+}\right)\left(\frac{\vec{\partial}}{\partial h_{i}^{+}} f\left(h, h^{+}\right)\right) \\
\int d^{2} \mathbf{h}\left(f\left(h, h^{+}\right) \frac{\overleftarrow{\partial}}{\partial h_{i}^{+}}\right) e\left(h, h^{+}\right) & =-\sigma(e) \int d^{2} \mathbf{h} f\left(h, h^{+}\right)\left(e\left(h, h^{+}\right) \frac{\overleftarrow{\partial}}{\partial h_{i}^{+}}\right)
\end{aligned}
$$

where $\sigma(e, f)=+1,-1$ depending on whether $e, f$ is even or odd. A similar result applies when each mode is associated with a complex pair of Grassmann variables $h_{i}$ and $h_{i}^{*}$. 


\section{Appendix B - Fermion Coherent States}

\subsection{Definitions}

The fermion coherent state introduced by Cahill and Glauber [22] are Grassmann vectors defined by:

$$
\begin{aligned}
\left|g, g^{*}\right\rangle & =\exp \left(\sum_{i}\left(\hat{c}_{i}^{\dagger} g_{i}-g_{i}^{*} \hat{c}_{i}\right)\right)|0\rangle \\
& =\prod_{i=1}^{n}\left[1+\hat{c}_{i}^{\dagger} g_{i}-g_{i}^{*} \hat{c}_{i}+\left(\hat{c}_{i}^{\dagger} \hat{c}_{i}-\frac{1}{2}\right) g_{i}^{*} g_{i}\right]|0\rangle .
\end{aligned}
$$

The fermion coherent state involves all $2 n$ Grassmann numbers $\left\{g, g^{*}\right\} \equiv g_{1} \cdots g_{n}, g_{1}^{*} \cdots g_{n}^{*}$ and can be written as a product of coherent states $\left|g_{i}, g_{i}^{*}\right\rangle$ for the separate modes

$$
\left|g, g^{*}\right\rangle=\left|g_{1}, g_{1}^{*} \cdots, g_{n}, g_{n}^{*}\right\rangle=\prod_{i}\left|g_{i}, g_{i}^{*}\right\rangle
$$

where

$$
\left|g_{i}, g_{i}^{*}\right\rangle=\exp \left(\hat{c}_{i}^{\dagger} g_{i}-g_{i}^{*} \hat{c}_{i}\right)\left|0_{i}\right\rangle
$$

and $\left|0_{i}\right\rangle$ is the vacuum state for the $i$ th mode. The vacuum state is itself a fermion coherent state with $g=g^{*}=0$. The fermion coherent state is normalised to unity. Note that the fermion coherent state is an example of an even Grassmann vector, in that each term in its expansion contains the products of pairs of anti-commuting operators or Grassmann variables. For an attempt to define fermion coherent states using c-numbers, see Tyc et al [51].

The fermion Bargmann coherent states are defined for a set of Grassmann numbers $g \equiv$ $\left\{g_{1}, g_{2}, \cdots, g_{i}, \cdots, g_{n}\right\}$ by

$$
\begin{aligned}
|g\rangle_{B} & =\exp \left(\sum_{i}\left(\hat{c}_{i}^{\dagger} g_{i}\right)\right)|0\rangle=\prod_{i}\left(1+\hat{c}_{i}^{\dagger} g_{i}\right)|0\rangle=\prod_{i}\left|g_{i}\right\rangle_{B} \\
& =\exp \left(\frac{1}{2} \mathbf{g}^{*} \cdot \mathbf{g}\right)\left|g, g^{*}\right\rangle
\end{aligned}
$$

where $\mathbf{g}^{*} \cdot \mathbf{g}=\sum_{i} g_{i}^{*} g_{i}$. This is also an even Grassmann vector, but now only depends on $g_{i}$ and not on the complex conjugates $g_{i}^{*}$. For the mode $\left|\phi_{i}\right\rangle$ the Bargmann coherent state is

$$
\left|g_{i}\right\rangle_{B}=\left(1+\hat{c}_{i}^{\dagger} g_{i}\right)|0\rangle=\left|0_{i}\right\rangle-g_{i}\left|1_{i}\right\rangle
$$

which is a superposition of the vacuum state and a one-fermion state.

\subsection{Properties}

The Bargmann coherent states are un-normalised versions of the fermion coherent states (171) and satisfy the following orthonormality result.

$$
{ }_{B}\langle g \mid h\rangle_{B}=\exp \left(\mathbf{g}^{*} \cdot \mathbf{h}\right)
$$


where $\mathbf{g}^{*} \cdot \mathbf{h}=\sum_{i} g_{i}^{*} h_{i}$. To show this result we have

$$
\begin{aligned}
{ }_{B}\langle g \mid h\rangle_{B} & =\left\langle 0\left|\prod_{i}\left(1+g_{i}^{*} \hat{c}_{i}\right) \prod_{j}\left(1+\hat{c}_{j}^{\dagger} h_{j}\right)\right| 0\right\rangle \\
& =\left\langle 0\left|\prod_{i}\left(1+g_{i}^{*} \hat{c}_{i}\right)\left(1+\hat{c}_{i}^{\dagger} h_{i}\right)\right| 0\right\rangle \\
& =\left\langle 0\left|\prod_{i}\left(1+g_{i}^{*} \hat{c}_{i}+\hat{c}_{i}^{\dagger} h_{i}+g_{i}^{*}\left\{1-\hat{c}_{i}^{\dagger} \hat{c}_{i}\right\} h_{i}\right)\right| 0\right\rangle \\
& =\prod_{i}\left(1+g_{i}^{*} h_{i}\right)=\exp \left(\mathbf{g}^{*} \cdot \mathbf{h}\right)
\end{aligned}
$$

where the anti-commutation rules and (8) have been used.

Bargmann coherent states are right-eigenstates of the annihilation operators $\hat{c}_{i}$ and the corresponding bra vectors are left eigenstates of the creation operators $\hat{c}_{i}^{\dagger}$

$$
\hat{c}_{i}|g\rangle_{B}=g_{i}|g\rangle_{B} . \quad\left\langle\left. g\right|_{B} \hat{c}_{i}^{\dagger}=\left\langle\left. g\right|_{B} g_{i}^{*}\right.\right.
$$

Unlike in boson coherent states the eigenvalues here are Grassmann numbers. To show this we have

$$
\begin{aligned}
\hat{c}_{i}|g\rangle_{B} & =\hat{c}_{i} \prod_{j}\left(1+\hat{c}_{j}^{\dagger} g_{j}\right)|0\rangle=\prod_{j \neq i}\left(1+\hat{c}_{j}^{\dagger} g_{j}\right)\left(\hat{c}_{i}+\left\{1-\hat{c}_{i}^{\dagger} \hat{c}_{i}\right\} g_{i}\right)|0\rangle \\
& =\prod_{j \neq i}\left(1+\hat{c}_{j}^{\dagger} g_{j}\right)\left\{g_{i}\right\}|0\rangle=\prod_{j \neq i}\left(1+\hat{c}_{j}^{\dagger} g_{j}\right)\left\{g_{i}\left(1+\hat{c}_{i}^{\dagger} g_{i}\right)\right\}|0\rangle \\
& =g_{i}|g\rangle_{B}
\end{aligned}
$$

where (174) and (8) have been used.

The scalar product of the Bargmann coherent state $|g\rangle_{B}$ with the Fock state $|\Phi\{l\}\rangle$ is given by

$$
\langle\Phi\{l\} \mid g\rangle_{B}=\left\langle 0\left|\left(\hat{c}_{l_{p}}\right) \cdots .\left(\hat{c}_{l_{2}}\right)\left(\hat{c}_{l_{1}}\right)\right| g\right\rangle_{B}=g_{l_{p}} \cdots . g_{l_{2}} g_{l_{1}}
$$

where (177) has been used with $g \rightarrow 0, h \rightarrow g$.

\subsection{Projectors}

The Bargmann states can be used the construct projectors of the form $|g\rangle_{B B}\langle h|$. The trace of the Bargmann state projectors are

$$
\operatorname{Tr}\left(|g\rangle_{B B}\langle h|\right)=\exp \left(\mathbf{g} \cdot \mathbf{h}^{*}\right)={ }_{B}\langle-h \mid g\rangle_{B}={ }_{B}\langle h \mid-g\rangle_{B},
$$

which is not the same as ${ }_{B}\langle h \mid g\rangle_{B}$ as it would be for the boson case. To show that result we evaluate the trace using Fock states

$$
\begin{aligned}
\operatorname{Tr}\left(|g\rangle_{B B}\langle h|\right) & =\sum_{\nu_{1} . . \nu_{i} . . \nu_{i n}}\left\langle\nu_{1}, ., \nu_{i}, \cdots \nu_{n}\left|\prod_{i}\right| g_{i}\right\rangle_{B}\left\langle\left. h_{i}\right|_{B} \mid \nu_{1}, ., \nu_{i}, \cdots \nu_{n}\right\rangle \\
& =\prod_{i} \sum_{\nu_{i} .=0,1}\left\langle\nu_{i} \mid g_{i}\right\rangle_{B B}\left\langle h_{i} \mid \nu_{i}\right\rangle \\
& =\prod_{i}\left\{\left\langle 0 \mid g_{i}\right\rangle_{B B}\left\langle h_{i} \mid 0\right\rangle+\left\langle 1 \mid g_{i}\right\rangle_{B B}\left\langle h_{i} \mid 1\right\rangle\right\} \\
& =\prod_{i}\left\{1+g_{i} h_{i}^{*}\right\}=\exp \left(\mathbf{g} \cdot \mathbf{h}^{*}\right)
\end{aligned}
$$

where (174) and (8) have been used. 
Normalised forms of the Bargmann state projectors are defined by

$$
\hat{\Lambda}_{f}\left(g, h^{*}\right)=\frac{|g\rangle_{B B}\langle h|}{\operatorname{Tr}\left(|g\rangle_{B B}\langle h|\right)}
$$

where the projectors have also been expressed in terms of Bargmann states. These normalised projectors are constructed so that their trace is unity:

$$
\operatorname{Tr}\left(\hat{\Lambda}_{f}\left(g, h^{*}\right)\right)=1 \text {. }
$$

Note that $\hat{\Lambda}_{f}\left(g, h^{*}\right)$ only depends on $g_{i}$ and $h_{i}^{*}$. These features are useful in constructing canonical forms of the density operator in terms of phase space distribution functions.

\subsection{Completeness}

The Bargmann coherent states satisfy a completeness relation, analogous to the result for boson Bargmann coherent states.

$$
\int \prod_{i} d g_{i}^{*} d g_{i} \exp \left(-\mathbf{g}^{*} \cdot \mathbf{g}\right)|g\rangle_{B}\left\langle\left. g\right|_{B}=\hat{1}\right.
$$

To show this important result we evaluate the matrix element of the left side $\hat{\Xi}$ between two general states $|\Phi\rangle$ and $|\Theta\rangle$ of the form (11), where $n$ is the number of modes and $l \equiv\left\{l_{1}, l_{2} \cdots l_{q}\right\}$ and $m \equiv\left\{m_{1}, m_{2} \cdots m_{p}\right\}$ lists two sets of occupied modes associated with expanding $|\Phi\rangle$ and $|\Theta\rangle$ in terms of Fock states. We have $0 \leq p, q \leq n$, and these modes will be listed in a conventional order - denoted $l_{1}<l_{2}<\cdots<l_{q}$. and $m_{1}<m_{2}<\cdots<m_{p}$. Starting with the form (11) for expanding the states we would have for ${ }_{B}\langle g \mid \Phi\rangle$

$$
\begin{aligned}
{ }_{B}\langle g \mid \Phi\rangle & =\sum_{\nu} B\left(\nu_{1} \cdots \nu_{n}\right) \prod_{i}\left(\left\langle 0_{i}\left|-\left\langle 1_{i}\right| g_{i}^{*}\right) \mid \nu_{i}\right\rangle\right. \\
& =\sum_{m} D\left(m_{1} \cdots m_{p}\right) g_{m_{1}}^{*} \cdots g_{m_{p}}^{*}
\end{aligned}
$$

where $D\left(m_{1} \cdots m_{p}\right)$ designate new expansion coefficients for $|\Phi\rangle$ in terms of the occupied Fock states $\left|m_{1} \cdots m_{p}\right\rangle$. Using (176) we then see that the scalar product $\left(\left\langle 0_{i}\left|-\left\langle 1_{i}\right| g_{i}^{*}\right) \mid \nu_{i}\right\rangle\right.$ equals 1 if mode $\left|\phi_{i}\right\rangle$ is unoccupied and equals $+g_{i}^{*}$ if it is occupied. A related expression applies for $\langle\Theta \mid g\rangle_{B}$, the expansion coefficients for occupied modes then being denoted as $C\left(l_{1} \cdots l_{q}\right)$. Taking the c-numbers outside the integral we find that

$$
\begin{aligned}
\langle\Theta|\hat{\Xi}| \Phi\rangle= & \int d^{2} \mathbf{g} \exp \left(-\mathbf{g}^{*} \cdot \mathbf{g}\right)\langle\Theta \mid g\rangle_{B}{ }_{B}\langle g \mid \Phi\rangle \\
= & \int d^{2} \mathbf{g} \exp \left(-\mathbf{g}^{*} \cdot \mathbf{g}\right) \sum_{\{l\}} C^{*}\left(l_{1} \cdots l_{q}\right) g_{l_{q}} \cdots g_{l_{1}} \\
\quad & \quad \sum_{\{m\}} D\left(m_{1} \cdots m_{p}\right) g_{m_{1}}^{*} \cdots g_{m_{p}}^{*} \\
= & \sum_{\{l\},\{m\}} C^{*}\left(l_{1} \cdots l_{q}\right) D\left(m_{1} \cdots m_{p}\right) \times \\
& \quad \times \int d^{2} \mathbf{g} \prod_{i}\left(1-g_{i}^{*} g_{i}\right) g_{l_{q}} \cdots g_{l_{1}} g_{m_{1}}^{*} \cdots g_{m_{p}}^{*} .
\end{aligned}
$$

where $d^{2} \mathbf{g} \equiv \prod_{i} d g_{i}^{*} d g_{i}$. These modes are listed in a conventional order - denoted $m_{1}<m_{2}<$ $\cdots<m_{p}$ and $l_{1}<l_{2}<\cdots<l_{q}$. If for a particular pair of sets $l, m$ a mode is occupied, then 
the $g_{i}^{*} g_{i}$ factor for one of these occupied modes will multiply a $g$ or $g^{*}$ present in the factor $g_{l_{q}} \cdots g_{l_{1}} g_{m_{1}}^{*} \cdots g_{m_{p}}^{*}$ and give zero. Hence the product of the $\left(1-g_{i}^{*} g_{i}\right)$ can be replaced by unity for modes in the sets $l, m$ giving

$$
\begin{aligned}
\langle\Theta|\hat{\Xi}| \Phi\rangle= & \sum_{\{l\},\{m\}} C^{*}\left(l_{1} \cdots l_{q}\right) B\left(m_{1} \cdots m_{p}\right) \\
& \times \int d^{2} \mathbf{g} \prod_{i \neq\{l\},\{m\}}\left(1-g_{i}^{*} g_{i}\right) g_{l_{q}} \cdots g_{l_{1}} g_{m_{1}}^{*} \cdots g_{m_{p}}^{*} .
\end{aligned}
$$

Then for these unoccupied modes the partial Grassmann integral gives

$$
\int d^{2} g_{i}\left(1-g_{i}^{*} g_{i}\right)=-\int d g_{i}^{*} d g_{i} g_{i}^{*} g_{i}=\int d g_{i}^{*} g_{i}^{*} \int d g_{i} g_{i}=1 .
$$

Note at this point an integal over both $g_{i}^{*}$ and $g_{i}$ is involved. Hence we have

$$
\langle\Theta|\hat{\Xi}| \Phi\rangle=\sum_{l, m} C^{*}\left(l_{1} \cdots l_{q}\right) D\left(m_{1} \cdots m_{p}\right) \int \prod_{k=l, m} d g_{k}^{*} d g_{k} g_{l_{q}} \cdots g_{l_{1}} g_{m_{1}}^{*} \cdots g_{m_{p}}^{*}
$$

where now the integration is only over modes in the $l, m$ sets.

We now can see that there is no contribution unless the two sets of occupied modes $l, m$ are the same. If that is not the case then there will be no matching pairs of $g_{l_{i}}$ and $g_{l_{i}}^{*}$ in the factor

$g_{l_{q}} \cdots g_{l_{2}} g_{l_{1}} g_{m_{1}}^{*} g_{m_{2}}^{*} \cdots g_{m_{p}}^{*}$ and then an integral of the form $\int d g_{i}^{*}$ or $\int d g_{i}$ will give zero. Hence $p=q$ and $l_{1}=m_{1} \cdots l_{p}=m_{p}$ and thus

$$
\langle\Theta|\hat{\Xi}| \Phi\rangle=\sum_{\{m\}} C^{*}\left(m_{1} \cdots m_{p}\right) D\left(m_{1} \cdots m_{p}\right) \int \prod_{k=m} d g_{k}^{*} d g_{k} g_{m_{p}} \cdots g_{m_{1}} g_{m_{1}}^{*} \cdots g_{m_{p}}^{*} .
$$

Finally we carry out the integrations in succession starting with $\int d g_{m_{1}}^{*} d g_{m_{1}}$ and find

$$
\begin{aligned}
& \int \prod_{k=m} d g_{k}^{*} d g_{k} g_{m_{p}} \cdots g_{m_{1}} g_{m_{1}}^{*} \cdots g_{m_{p}}^{*} \\
= & \int \prod_{k=m, k \neq m_{1}} d g_{k}^{*} d g_{k} g_{m_{p}} \cdots g_{m_{2}} g_{m_{2}}^{*} \cdots g_{m_{p}}^{*} \times 1 \\
= & 1
\end{aligned}
$$

after all the integrals have been done. Hence we have found that

$$
\langle\Theta|\hat{\Xi}| \Phi\rangle=\sum_{\{m\}} C^{*}\left(m_{1} \cdots m_{p}\right) D\left(m_{1} \cdots m_{p}\right)=\langle\Theta \mid \Phi\rangle
$$

so that the operator $\hat{\Xi}$ must be the identity:

$$
\int d^{2} \mathbf{g} \exp \left(-\mathbf{g}^{*} \cdot \mathbf{g}\right)|g\rangle_{B}\left\langle g\left|=\int d^{2} \mathbf{g}\right| g, g^{*}\right\rangle\left\langle g, g^{*}\right|=\hat{1} .
$$

where the second expression expresses the completeness relation in terms of the Glauber fermion coherent states $\left|g, g^{*}\right\rangle$.

\section{Appendix C - Canonical Form of Density Operator}

We can write any fermionic operator as a linear combination of coherent-state projectors by pre- and post-multiplying by the identity set out in Eq.(186) 


$$
\begin{aligned}
\hat{\rho} & =\int d^{2} \mathbf{g} e^{-\mathbf{g}^{*} \cdot \mathbf{g}}|g\rangle_{B B}\left\langle g\left|\hat{\rho}_{f} \int d^{2} \mathbf{h} e^{-\mathbf{h}^{*} \cdot \mathbf{h}}\right| h\right\rangle_{B B}\langle h| \\
& =\int d^{2} \mathbf{g} d^{2} \mathbf{h}|g\rangle_{B B}\langle h| e^{-\mathbf{g}^{*} \cdot \mathbf{g}-\mathbf{h}^{*} \cdot \mathbf{h}} \rho\left(g^{*}, h\right) .
\end{aligned}
$$

where $d^{2} \mathbf{g}=\prod_{i} d g_{i}^{*} d g_{i}$ and $\mathbf{h}^{*} \cdot \mathbf{h}=\sum_{i} h_{i}^{*} h_{i}$ etc. Here we have used the facts that the differentials $d^{2} h_{i}$ commute with $\hat{\rho}$ and with the even Grassmann quantities ${ }_{B}\langle g|$ and $|g\rangle_{B}$ and also that the Grassmann function ${ }_{B}\langle g|\hat{\rho}| h\rangle_{B}=\rho\left(g^{*}, h\right)$ commutes with the even quantity ${ }_{B}\langle h|$. We arrive at the canonical form for the density operator $\hat{\rho}$ by making the change of Grassmann variable notation $h \rightarrow g^{+*}$ so that

$$
\hat{\rho}=\int d^{2} \mathbf{g} d^{2} \mathbf{g}^{+*}|g\rangle_{B B}\left\langle g^{+*}\right| e^{-\mathbf{g}^{*} \cdot \mathbf{g}-\mathbf{g}^{+} \cdot \mathbf{g}^{+*}} \rho\left(g^{*}, g^{+*}\right),
$$

where $d^{2} \mathbf{g}^{+*}=\prod_{i} d g_{i}^{+} d g_{i}^{+*}$. Although the order of the pairs of differentials in each of $d^{2} \mathbf{g}$ and $d^{2} \mathbf{g}^{+*}$ is arbitrary, we choose the order to be $1,2, \cdots, n$. Using (136) twice we can write after canceling the two $(-1)^{n(n-1) / 2}$ factors

$$
\begin{aligned}
d^{2} \mathbf{g} d^{2} \mathbf{g}^{+*} & =\prod_{i} d g_{i}^{*} \prod_{j} d g_{j} \prod_{k} d g_{k}^{+} \prod_{l} d g_{l}^{+*} \\
& =\prod_{k} d g_{k}^{+} \prod_{j} d g_{j} \prod_{l} d g_{l}^{+*} \prod_{i} d g_{i}^{*}
\end{aligned}
$$

where the second line is obtained by using (137) twice and canceling the two $(-1)^{n^{2}}$ factors. We can then reverse the order of the $d g_{j}$ and the $d g_{i}^{*}$ to now be $n, \cdots, 2,1$ using (135) twice, and then after canceling the two $(-1)^{n(n-1) / 2}$ factors we finally have

$$
d^{2} \mathbf{g} d^{2} \mathbf{g}^{+*}=d \mathbf{g}^{+} d \mathbf{g} d \mathbf{g}^{+*} d \mathbf{g}^{*}
$$

with $d \mathbf{g}^{+}=\prod_{i} d g_{i}^{+}=d g_{1}^{+} d g_{2}^{+} \cdots d g_{n}^{+}, d \mathbf{g}=\prod_{i} d g_{i}=d g_{n} \cdots d g_{2} d g_{1}, d \mathbf{g}^{+*}=\prod_{i} d g_{i}^{+*}=d g_{1}^{+*} d g_{2}^{+*} \cdots d g_{n}^{+*}$, $d \mathbf{g}^{*}=\prod_{i} d g_{i}^{*}=d g_{n}^{*} \cdots d g_{2}^{*} d g_{1}^{*}$.

Using (199) and commuting even Grassmann factors we can rewrite the density operator in terms of the normalized Bargmann state projector

$$
\Lambda_{f}\left(g \cdot g^{+}\right)=\frac{|g\rangle_{B B}\left\langle g^{+*}\right|}{\operatorname{Tr}\left(|g\rangle_{B B}\left\langle g^{+*}\right|\right)}=e^{-\mathbf{g} \cdot \mathbf{g}^{+}}|g\rangle_{B B}\left\langle g^{+*}\right|
$$

in the form

$$
\hat{\rho}=\int d \mathbf{g}^{+} d \mathbf{g} P\left(g, g^{+}\right) \hat{\Lambda}\left(g, g^{+}\right)=\int d \mathbf{g}^{+} d \mathbf{g} \hat{\Lambda}\left(g, g^{+}\right) P\left(g, g^{+}\right)
$$

where

$$
\begin{aligned}
P\left(g, g^{+}\right) & =\int d \mathbf{g}^{+*} d \mathbf{g}^{*} e^{\mathbf{g} \cdot \mathbf{g}^{*}+\mathbf{g}^{+*} \cdot \mathbf{g}^{+}+\mathbf{g} \cdot \mathbf{g}^{+}} \rho\left(g^{*}, g^{+*}\right) \\
& =\int d \mathbf{g}^{+*} d \mathbf{g}^{*} e^{\mathbf{g} \cdot \mathbf{g}^{*}+\mathbf{g}^{+*} \cdot \mathbf{g}^{+}+\mathbf{g} \cdot \mathbf{g}^{+}}{ }_{B}\left\langle g|\hat{\rho}| g^{+*}\right\rangle_{B}
\end{aligned}
$$

is the canonical representation of the fermion density operator $\hat{\rho}$. Here $d \mathbf{g}=\prod_{i} d g_{i}$ and $d \mathbf{g}^{+}=\prod_{i} d g_{i}^{+}$etc. Note that an analogous representation is valid for any fermion operator. 


\section{Appendix D - Quantum Correlation Function and Fock State Populations, Coherences}

\subsection{Quantum Correlation Function - $P$ Distribution}

The simplest derivation just involves substituting the canonical form (21) of the density operator into the definition (27). We have

$$
\begin{aligned}
& \left\langle\left(\hat{c}_{l_{1}}^{\dagger}\right)\left(\hat{c}_{l_{2}}^{\dagger}\right) . .\left(\hat{c}_{l_{p}}^{\dagger}\right)\left(\hat{c}_{m_{q}}\right) . .\left(\hat{c}_{m_{2}}\right)\left(\hat{c}_{m_{1}}\right)\right\rangle \\
= & \operatorname{Tr}\left\{\left(\hat{c}_{m_{q}}\right) . .\left(\hat{c}_{m_{2}}\right)\left(\hat{c}_{m_{1}}\right) \hat{\rho}\left(\hat{c}_{l_{1}}^{\dagger}\right)\left(\hat{c}_{l_{2}}^{\dagger}\right) . .\left(\hat{c}_{l_{p}}^{\dagger}\right)\right\} \\
= & \int \prod_{i} d g_{i}^{+} d g_{i} P\left(g, g^{+}\right) \frac{1}{\operatorname{Tr}\left(|g\rangle_{B}\left\langle\left. g^{+*}\right|_{B}\right)\right.} \\
& \times \operatorname{Tr}\left\{\left(\hat{c}_{m_{q}}\right) . .\left(\hat{c}_{m_{2}}\right)\left(\hat{c}_{m_{1}}\right)|g\rangle_{B}\left\langle\left. g^{+*}\right|_{B}\left(\hat{c}_{l_{1}}^{\dagger}\right)\left(\hat{c}_{l_{2}}^{\dagger}\right) . .\left(\hat{c}_{l_{p}}^{\dagger}\right)\right\}\right.
\end{aligned}
$$

where the even number of differentials commute with the mode annihilation operators and the even Grassmann functions $P\left(g, g^{+}\right)$and $1 / \operatorname{Tr}\left(|g\rangle_{B}\left\langle\left. g^{+*}\right|_{B}\right)\right.$, which then all can be taken outside the trace process. Using the eigenvalue equations (179) for the Bargmann coherent states we then have

$$
\begin{aligned}
& \operatorname{Tr}\left\{\left(\hat{c}_{m_{q}}\right) . .\left(\hat{c}_{m_{2}}\right)\left(\hat{c}_{m_{1}}\right)|g\rangle_{B}\left\langle\left. g^{+*}\right|_{B}\left(\hat{c}_{l_{1}}^{\dagger}\right)\left(\hat{c}_{l_{2}}^{\dagger}\right) . .\left(\hat{c}_{l_{p}}^{\dagger}\right)\right\}\right. \\
= & \operatorname{Tr}\left\{\left(g_{m_{q}}\right) . .\left(g_{m_{2}}\right)\left(g_{m_{1}}\right)|g\rangle_{B}\left\langle\left. g^{+*}\right|_{B}\left(g_{l_{1}}^{+}\right)\left(g_{l_{2}}^{+}\right) . .\left(g_{l_{p}}^{+}\right)\right\}\right.
\end{aligned}
$$

Then assuming that $p-q$ is an even number, there are an even number of Grassmann variables and these can be taken outside the trace process. The factors $\operatorname{Tr}\left(|g\rangle_{B}\left\langle\left. g^{+*}\right|_{B}\right)\right.$ then cancel giving the result

$$
\begin{aligned}
& \left\langle\left(\hat{c}_{l_{1}}^{\dagger}\right)\left(\hat{c}_{l_{2}}^{\dagger}\right) . .\left(\hat{c}_{l_{p}}^{\dagger}\right)\left(\hat{c}_{m_{q}}\right) . .\left(\hat{c}_{m_{2}}\right)\left(\hat{c}_{m_{1}}\right)\right\rangle \\
= & \int \prod_{i} d g_{i}^{+} d g_{i} \times\left(g_{m_{q}}\right) . .\left(g_{m_{2}}\right)\left(g_{m_{1}}\right) P\left(g, g^{+}\right)\left(g_{l_{1}}^{+}\right)\left(g_{l_{2}}^{+}\right) . .\left(g_{l_{p}}^{+}\right)
\end{aligned}
$$

which is valid when $p-q$ is even.

\subsection{Normalisation Integrals}

The normalisation integral for the $P\left(g, g^{+}\right)$distribution function can be obtained from $\operatorname{Tr}\{\hat{\rho}\}=1$, and from (205) with all mode operators and phase space variables absent we find that

$$
\int \prod_{i} d g_{i}^{+} d g_{i} P\left(g, g^{+}\right)=1
$$

The normalisation for the $B\left(g, g^{+}\right)$distribution function can then be obtained using $(25)$ and is

$$
\int \prod_{i} d g_{i}^{+} d g_{i} \exp \left(+\mathbf{g} \cdot \mathbf{g}^{+}\right) B\left(g, g^{+}\right)=1
$$




\subsection{Fock State Populations and Coherences - $B$ Distribution}

To determine the population we substitute for the $\hat{\rho}$ from (26) to give - using the notation $d \mathbf{g}^{+} d \mathbf{g}=\prod_{i} d g_{i}^{+} d g_{i}$

$$
\begin{aligned}
P(\Phi\{l\}) & =\operatorname{Tr}\left(\int d \mathbf{g}^{+} d \mathbf{g} B\left(g, g^{+}\right)(|\Phi\{l\}\rangle\langle\Phi\{l\}|)\left(|g\rangle_{B}\left\langle\left. g^{+*}\right|_{B}\right)\right)\right. \\
& =\operatorname{Tr}\left(\int d \mathbf{g}^{+} d \mathbf{g} B\left(g, g^{+}\right) \hat{c}_{l_{1}}^{\dagger} \cdots \hat{c}_{l_{p}}^{\dagger}|0\rangle\langle 0| g_{l_{p}} \cdots g_{l_{1}}\left(|g\rangle_{B}\left\langle\left. g^{+*}\right|_{B}\right)\right)\right. \\
& =\operatorname{Tr}\left(\int d \mathbf{g}^{+} d \mathbf{g} B\left(g, g^{+}\right)(-1)^{p} g_{l_{p}} \cdots g_{l_{1}} \hat{c}_{l_{1}}^{\dagger} \cdots \hat{c}_{l_{p}}^{\dagger}|0\rangle\left\langle\left. g^{+*}\right|_{B}\right)\right.
\end{aligned}
$$

since as $|\Phi\{l\}\rangle\langle\Phi\{l\}|$ is an even operator it commutes with the product of the Grassmann differentials and with the even function $B\left(g, g^{+}\right)$, enabling the fermion annihilation operators to act on $|g\rangle_{B}$ to give a product of eigenvalues $g_{l_{p}} \cdots g_{l_{1}}$. This product then commutes with the same number of fermion creation operators with a factor $(-1)^{p}$ and the factor $\langle 0 \mid g\rangle_{B}$ is equal to unity. Then evaluating the trace using Fock states $\left|\nu_{1} \nu_{2} \cdots \nu_{n}\right\rangle$ and noting that this Fock state is even or odd according to whether $(-1)^{\nu}=+1,-1\left(\nu=\sum_{i} \nu_{i}\right)$ we can commute the bra vector $\left\langle\nu_{1} \nu_{2} \cdots \nu_{n}\right|$ with the even product of Grassmann differentials, the even $B\left(g, g^{+}\right)$and the product $g_{l_{p}} \cdots g_{l_{1}}$ apart from a factor $(-1)^{\nu}$ if $p$ is odd. Thus

$$
\begin{aligned}
P(\Phi\{l\}) & =\int d \mathbf{g}^{+} d \mathbf{g} B\left(g, g^{+}\right)(+1) g_{l_{p}} \cdots g_{l_{1}} \\
& \times \sum_{\{\nu\}}\left\langle\nu_{1} \cdots \nu_{n}\left|\hat{c}_{l_{1}}^{\dagger} \cdots \hat{c}_{l_{p}}^{\dagger}\right| 0\right\rangle_{B}\left\langle g^{+*} \mid \nu_{1} \cdots \nu_{n}\right\rangle \quad \text { (p even) } \\
& =\int d \mathbf{g}^{+} d \mathbf{g} B\left(g, g^{+}\right)(-1) g_{l_{p}} \cdots g_{l_{1}} \\
& \times \sum_{\{\nu\}}(-1)^{\nu}\left\langle\nu_{1} \cdots \nu_{n}\left|\hat{c}_{l_{1}}^{\dagger} \cdots \hat{c}_{l_{p}}^{\dagger}\right| 0\right\rangle_{B}\left\langle\mathbf{g}^{+*} \mid \nu_{1} \cdots \nu_{n}\right\rangle \quad(\mathrm{p} \text { odd })
\end{aligned}
$$

where we have inserted the result for $(-1)^{p}$ in the two cases. In the case where $p$ is odd, we then use ${ }_{B}\left\langle g^{+*} \mid \nu_{1} \cdots \nu_{n}\right\rangle=(-1)_{B}^{\nu}\left\langle-g^{+*} \mid \nu_{1} \cdots \nu_{n}\right\rangle$ from Eq.(181) which allows the two $(-1)^{\nu}$ to cancel out. We then reverse the order of the c-number $\left\langle\nu_{1} \cdots \nu_{n}\left|\hat{c}_{l_{1}}^{\dagger} \cdots \hat{c}_{l_{p}}^{\dagger}\right| 0\right\rangle$ and the Grassmann function $\left\langle-g^{+*} \mid \nu_{1} \cdots \nu_{n}\right\rangle$ and apply the result $\sum_{\{\nu\}}\left|\nu_{1} \cdots \nu_{n}\right\rangle\left\langle\nu_{1} \cdots \nu_{n}\right|=\hat{1}$. When $p$ is even, no factor $(-1)^{\nu}$ occurs, so we just reverse the order of the c-number $\left\langle\nu_{1} \cdots \nu_{n}\left|\hat{c}_{l_{1}}^{\dagger} \cdots \hat{c}_{l_{p}}^{\dagger}\right| 0\right\rangle$ and the Grassmann function ${ }_{B}\left\langle g^{+*} \mid \nu_{1} \nu_{2} \cdots \nu_{n}\right\rangle$ and apply the completeness result. Hence we find that

$$
\begin{aligned}
& P(\Phi\{l\})=\int d \mathbf{g}^{+} d \mathbf{g} B\left(g, g^{+}\right)(+1) g_{l_{p}} \cdots g_{l_{1}}\left\langle\left. g^{+*}\right|_{B} \hat{c}_{l_{1}}^{\dagger} \cdots \hat{c}_{l_{p}}^{\dagger} \mid 0\right\rangle \quad \text { (p even) } \\
& =\int d \mathbf{g}^{+} d \mathbf{g} B\left(g, g^{+}\right)(-1) g_{l_{p}} \cdots g_{l_{1}}\left\langle-\left.g^{+*}\right|_{B} \hat{c}_{l_{1}}^{\dagger} \cdots \hat{c}_{l_{p}}^{\dagger} \mid 0\right\rangle \quad(\mathrm{p} \text { odd })
\end{aligned}
$$

If $p$ is odd, using the eigenvalue equation for $\left\langle-\left.g^{+*}\right|_{B}\right.$ leads to a further product of Grassmann variables $g_{l_{1}}^{+} \cdots g_{l_{p}}^{+}$times $(-1)$. If $p$ is even, using the eigenvalue equation for $\left\langle\left. g^{+*}\right|_{B}\right.$ leads to the same product of Grassmann variables but with a $(+1)$ factor. As ${ }_{B}\left\langle \pm g^{+*} \mid 0\right\rangle=1$ and noting that the two $(-1)$ cancel for the odd $p$ case, we finally get the result

$$
P(\Phi\{l\})=\int d \mathbf{g}^{+} d \mathbf{g} B\left(g, g^{+}\right) g_{l_{p}} \cdots g_{l_{1}} g_{l_{1}}^{+} \cdots g_{l_{p}}^{+}
$$


for the population of the fermion Fock state $|\Phi\{l\}\rangle=\hat{c}_{l_{1}}^{\dagger} \cdots \hat{c}_{l_{P}}^{\dagger}|0\rangle$ for both cases of $p$ even or odd. A similar treatment gives the result for the coherence

$$
C(\Phi\{m\} ; \Phi\{l\})=\int d \mathbf{g}^{+} d \mathbf{g} B\left(g, g^{+}\right) g_{l_{p}} \cdots g_{l_{1}} g_{m_{1}}^{+} \cdots g_{m_{p}}^{+}
$$

In both cases a phase space average with the un-normalised distribution function $B\left(g, g^{+}\right)$of a product of the Grassmann variables associated with the occupied modes is involved. The results are analogous to the quantum correlation function expressions in terms of the normalised distribution function $P\left(g, g^{+}\right)$.

\section{Appendix E - Correspondence Rules}

\section{1 $B$ Distribution Function}

We will derive two correspondence rules, one involving differentiation the other not. The other proofs are similar.

If $\hat{\rho}$ is replaced by $\hat{c}_{i} \hat{\rho}$ then substituting for the density operator from (26) we have

$$
\begin{aligned}
\hat{c}_{i} \hat{\rho} & =\int \prod_{i} d g_{i}^{+} \prod_{i} d g_{i} B\left(g, g^{+}\right)\left(\hat{c}_{i}|g\rangle_{B}\left\langle\left. g^{+*}\right|_{B}\right)\right. \\
& =\int \prod_{i} d g_{i}^{+} \prod_{i} d g_{i}\left(g_{i} B\left(g, g^{+}\right)\right)\left(|g\rangle_{B}\left\langle\left. g^{+*}\right|_{B}\right)\right.
\end{aligned}
$$

since $\hat{c}_{i}$ commutes with the product of an even number of Grassmann differentials and with the even $B$ distribution, and $\hat{c}_{i}|g\rangle_{B}=g_{i}|g\rangle_{B}$. Thus $B$ is replaced by $g_{i} B$.

If $\hat{\rho}$ is replaced by $\hat{\rho} \hat{c}_{i}$ then substituting for the density operator from (26) we have

$$
\begin{aligned}
\hat{\rho} \hat{c}_{i} & =\int \prod_{i} d g_{i}^{+} \prod_{i} d g_{i} B\left(g, g^{+}\right)\left(|g\rangle_{B}\left\langle\left. g^{+*}\right|_{B} \hat{c}_{i}\right)\right. \\
& =\int \prod_{i} d g_{i}^{+} \prod_{i} d g_{i} B\left(g, g^{+}\right)\left(|g\rangle_{B}\left\{\left\langle\left. g^{+*}\right|_{B}\left(-\frac{\overleftarrow{\partial}}{\partial g_{i}^{+}}\right)\right\}\right)\right. \\
& =-\int \prod_{i} d g_{i}^{+} \prod_{i} d g_{i} B\left(g, g^{+}\right)\left(\left\{|g\rangle_{B}\left\langle\left. g^{+*}\right|_{B}\right\}\left(\frac{\overleftarrow{\partial}}{\partial g_{i}^{+}}\right)\right)\right. \\
& =+\int \prod_{i} d g_{i}^{+} \prod_{i} d g_{i}\left\{B\left(g, g^{+}\right)\left(\frac{\overleftarrow{\partial}}{\partial g_{i}^{+}}\right)\right\}\left(|g\rangle_{B}\left\langle\left. g^{+*}\right|_{B}\right)\right.
\end{aligned}
$$

where $\left\langle\left. g^{+*}\right|_{B} \hat{c}_{i}==\left\langle\left. g^{+*}\right|_{B}\left(-\frac{\overleftarrow{\partial}}{\partial g_{i}^{+}}\right)\right.\right.$and the second integration by parts result in (170) is used noting that $|g\rangle_{B}\left\langle\left. g^{+*}\right|_{B}\right.$ is even. Thus $B$ is replaced by $B\left(+\frac{\overleftarrow{\partial}}{\partial g_{i}^{+}}\right)$.

As an illustration of applying the rules in succession consider replacing $\hat{\rho}$ by $\hat{c}_{j}^{\dagger} \hat{\rho} \hat{c}_{i}$. We have 
using the last equation and noting that $B\left(+\frac{\overleftarrow{\partial}}{\partial g_{i}^{+}}\right)$is odd

$$
\begin{aligned}
\hat{c}_{j}^{\dagger} \hat{\rho} \hat{c}_{i} & =-\int \prod_{i} d g_{i}^{+} \prod_{i} d g_{i}\left\{B\left(g, g^{+}\right)\left(\frac{\overleftarrow{\partial}}{\partial g_{i}^{+}}\right)\right\}\left(\hat{c}_{j}^{\dagger}|g\rangle_{B}\left\langle\left. g^{+*}\right|_{B}\right)\right. \\
& =-\int \prod_{i} d g_{i}^{+} \prod_{i} d g_{i}\left\{B\left(g, g^{+}\right)\left(\frac{\overleftarrow{\partial}}{\partial g_{i}^{+}}\right)\right\}\left(\left\{\left(-\frac{\partial}{\partial g_{j}}\right)|g\rangle_{B}\right\}\left\langle\left. g^{+*}\right|_{B}\right)\right. \\
& =+\int \prod_{i} d g_{i}^{+} \prod_{i} d g_{i}\left\{B\left(g, g^{+}\right)\left(\frac{\overleftarrow{\partial}}{\partial g_{i}^{+}}\right)\right\}\left(\left(\frac{\vec{\partial}}{\partial g_{j}}\right)\left\{|g\rangle_{B}\left\langle\left. g^{+*}\right|_{B}\right\}\right)\right. \\
& =-\int \prod_{i} d g_{i}^{+} \prod_{i} d g_{i}\left(\left(\frac{\partial}{\partial g_{j}}\right)\left\{|g\rangle_{B}\left\langle\left. g^{+*}\right|_{B}\right\}\right)\left\{B\left(g, g^{+}\right)\left(\frac{\overleftarrow{\partial}}{\partial g_{i}^{+}}\right)\right\}\right. \\
& =+\int \prod_{i} d g_{i}^{+} \prod_{i} d g_{i}\left(\left\{|g\rangle_{B}\left\langle\left. g^{+*}\right|_{B}\right\}\right)\left(\frac{\vec{\partial}}{\partial g_{j}}\right)\left\{B\left(g, g^{+}\right)\left(\frac{\overleftarrow{\partial}}{\partial g_{i}^{+}}\right)\right\}\right. \\
& =+\int \prod_{i} d g_{i}^{+} \prod_{i} d g_{i}\left\{\left(\frac{\vec{\partial}}{\partial g_{j}}\right) B\left(g, g^{+}\right)\left(\frac{\overleftarrow{\partial}}{\partial g_{i}^{+}}\right)\right\}\left(\left\{|g\rangle_{B}\left\langle\left. g^{+*}\right|_{B}\right\}\right)\right.
\end{aligned}
$$

where $\hat{c}_{j}^{\dagger}|g\rangle_{B}=\left(-\frac{\vec{\partial}}{\partial g_{i}}\right)|g\rangle_{B}$ and the first integration by parts result in (170) is used noting that $|g\rangle_{B}\left\langle\left. g^{+*}\right|_{B}\right.$ is even. Thus $B$ is replaced by $\left(+\frac{\vec{\partial}}{\partial g_{j}}\right) B\left(+\frac{\overleftarrow{\partial}}{\partial g_{i}^{+}}\right)$. The same result is obtained if we apply the process by first replacing $\hat{\rho}$ with $\hat{c}_{j}^{\dagger} \hat{\rho}$.

\section{2 $P$ Distribution Function}

The corresondence rules for the $P$ distribution function can be derived from those for the $B$ distribution function noting that $P\left(g, g^{+}\right)=B\left(g, g^{+}\right) \exp \left(+\mathbf{g} \cdot \mathbf{g}^{+}\right)$. We have

$$
\begin{aligned}
& \hat{\rho} \Rightarrow \hat{c}_{i} \hat{\rho} \quad P\left(g, g^{+}\right) \Rightarrow g_{i} P\left(g, g^{+}\right) \\
& \hat{\rho} \Rightarrow \hat{\rho} \hat{c}_{i} \quad P\left(g, g^{+}\right) \Rightarrow P\left(g, g^{+}\right)\left(+\frac{\overleftarrow{\partial}}{\partial g_{i}^{+}}-g_{i}\right) \\
& \hat{\rho} \Rightarrow \hat{c}_{i}^{\dagger} \hat{\rho} \quad P\left(g, g^{+}\right) \Rightarrow\left(+\frac{\vec{\partial}}{\partial g_{i}}-g_{i}^{+}\right) P\left(g, g^{+}\right) \\
& \hat{\rho} \Rightarrow \hat{\rho} \hat{c}_{i}^{\dagger} \quad P\left(g, g^{+}\right) \Rightarrow P\left(g, g^{+}\right) g_{i}^{+}
\end{aligned}
$$

\section{Appendix F - Fokker-Planck and Ito Stochastic Equations - $P$ Distribution Function}

\subsection{Fokker- Planck Equation}

This is of the same form as for the $B$ distribution

$$
\frac{\partial}{\partial t} P\left(g, g^{+}\right)=-\sum_{p=1}^{2 n}\left(A_{p} P\right) \frac{\overleftarrow{\partial}}{\partial g_{p}}+\frac{1}{2} \sum_{p, q=1}^{2 n}\left(D_{p q} P\right) \frac{\overleftarrow{\partial}}{\partial g_{q}} \frac{\overleftarrow{\partial}}{\partial g_{p}}
$$

where as before 


$$
\begin{gathered}
{[A]=\left[\begin{array}{c}
C^{-} \\
C^{+}
\end{array}\right]} \\
{[D]=\left[\begin{array}{cc}
-F^{--} & +F^{-+} \\
-\left(F^{-+}\right)^{T} & +F^{++}
\end{array}\right]}
\end{gathered}
$$

but now with

$$
\begin{aligned}
C_{i}^{-}=-\frac{1}{i \hbar} \sum_{j} h_{i j} g_{j}+\frac{1}{i \hbar} \sum_{j k l} \nu_{i j k l} g_{j}^{+} g_{l} g_{k}+\sum_{j k l}\left[\frac{\left(\Gamma_{j k i l}-\Gamma_{l i k j}\right)}{2} g_{l} g_{k}^{+}+\frac{\Gamma_{l i k j}}{2} \delta_{l k}\right] g_{j} \\
C_{i}^{+}=\frac{1}{i \hbar} \sum_{j} h_{i j}^{*} g_{j}^{+}-\frac{1}{i \hbar} \sum_{j k l} \nu_{i j k l}^{*} g_{k}^{+} g_{l}^{+} g_{j}+\sum_{j k l} g_{j}^{+}\left[\frac{\left(\Gamma_{j k i l}^{*}-\Gamma_{l i k j}^{*}\right)}{2} g_{k} g_{l}^{+}+\frac{\Gamma_{l i k j}^{*}}{2} \delta_{l k}\right] \\
F_{i j}^{--}=\frac{1}{i \hbar} \sum_{k l} \nu_{i j k l} g_{l} g_{k}+\sum_{k l} \frac{\left\{\Gamma_{l i j k}+\Gamma_{k j i l}\right\}}{2} g_{l} g_{k} \\
F_{i j}^{++}=-\frac{1}{i \hbar} \sum_{k l} \nu_{i j k l}^{*} g_{k}^{+} g_{l}^{+}+\sum_{k l} \frac{\left\{\Gamma_{l i j k}^{*}+\Gamma_{k j i l}^{*}\right\}}{2} g_{k}^{+} g_{l}^{+} \\
F_{i j}^{-+}=\sum_{k l}\left\{\Gamma_{j k i l}\right\} g_{l} g_{k}^{+} \quad F_{i j}^{+-}=\sum_{k l}\left\{\Gamma_{j k i l}^{*}\right\} g_{l}^{+} g_{k}
\end{aligned}
$$

Once again the drift vector is an odd Grassmann function and the diffusion matrix is even. However, whilst the diffusion matrix still depends quadratically on the Grassmann variables, the drift vector now has cubic terms. In fact the drift vector and diffusion matrix is the same as for the $B$ distribution case except for the addtional cubic terms in the drift vector - which depend on both two body interaction and relaxation terms. The diffusion matrix is anti-symmetric $D_{q p}=-D_{p q}$, as in the $B$ distribution case.

\subsection{Ito Stochastic Equations}

These are of the same form as for the $B$ distribution and are

$$
\frac{d}{d t} \tilde{g}_{p}(t)=C^{p}(\tilde{g}(t))+\sum_{a} B_{a}^{p}(\tilde{g}(t)) \Gamma_{a}\left(t_{+}\right)
$$

and the equations linking the classical and noise terms to the drift and diffusion terms in the Fokker-Planck equation are as before:

$$
\begin{aligned}
C^{p}\left(g, g^{+}\right) & =-A_{p}\left(g, g^{+}\right) \\
{\left[B\left(g, g^{+}\right) B^{T}\left(g, g^{+}\right)\right]_{q p} } & =D_{q p}\left(g, g^{+}\right)
\end{aligned}
$$

\section{Appendix G - Stochastic Average Time Derivative}

\subsection{Properties of Stochastic Averages}

Stochastic averages of sums and products can also be defined and these have important properties needed for evaluating expressions. For the sums of functions $F_{a}\left(\tilde{g}(t), \tilde{g}^{+}(t)\right)$ we have

$$
\overline{\sum_{a} F_{a}\left(\tilde{g}(t), \tilde{g}^{+}(t)\right)}=\sum_{a} \overline{F_{a}\left(\tilde{g}(t), \tilde{g}^{+}(t)\right)},
$$


so the average of the sum is the sum of the averages. The average of a product $\left.\left.F\left(\tilde{g}(t), \tilde{g}^{+}(t)\right)\right) E\left(\tilde{g}(t), \tilde{g}^{+}(t)\right)\right)$ is also important and is given by

$$
\overline{F\left(\tilde{g}(t), \tilde{g}^{+}(t)\right) E\left(\tilde{g}(t), \tilde{g}^{+}(t)\right)}=\frac{1}{m} \sum_{k=1}^{m} f\left(\tilde{g}_{p k}(t)\right) e\left(\tilde{g}_{p k}(t)\right) .
$$

Combining this result with that for a sum we find that the average of the sum of products is the sum of the average of each product

$$
\overline{\sum_{a}\left(F_{a}\left(\tilde{g}(t), \tilde{g}^{+}(t)\right) E_{a}\left(\tilde{g}(t), \tilde{g}^{+}(t)\right)\right)}=\sum_{a} \overline{\left(F_{a}\left(\tilde{g}(t), \tilde{g}^{+}(t)\right) E_{a}\left(\tilde{g}(t), \tilde{g}^{+}(t)\right)\right.} .
$$

The case where the quantities $F, E$ are uncorrelated is when the average of the product equals the product of the averages

$$
\overline{F\left(\tilde{g}(t), \tilde{g}^{+}(t)\right) E\left(\tilde{g}(t), \tilde{g}^{+}(t)\right)}=\overline{F\left(\tilde{g}(t), \tilde{g}^{+}(t)\right)} \overline{E\left(\tilde{g}(t), \tilde{g}^{+}(t)\right)}
$$

and obviously some special feature of the quasi-distribution function and the functions is required for this to occur.

\subsection{Time Derivative Derivation}

To derive the time derivative of the stochastic average of $F\left(\tilde{g}(t), \tilde{g}^{+}(t)\right)$ we consider the difference in the stochastic average from times $t$ to $t+\delta t$ which is given by

$$
\begin{aligned}
&= \overline{F\left(\tilde{g}(t+\delta t), \tilde{g}^{+}(t+\delta t)\right)}-\overline{F\left(\tilde{g}(t), \tilde{g}^{+}(t)\right)} \\
&= \frac{\left\{f\left(\tilde{g}(t+\delta t), \tilde{g}^{+}(t+\delta t)\right)-F\left(\tilde{g}(t), \tilde{g}^{+}(t)\right)\right\}}{\left.\left\{\tilde{g}_{p i}(t+\delta t)\right)-f\left(\tilde{g}_{p i}(t)\right)\right\}} \\
&=\left\{\sum_{p}\left[F\left(g, g^{+}\right) \frac{\overleftarrow{\partial}}{\partial g_{p}}\right] \delta \tilde{g}_{p}(t)+\frac{1}{2} \sum_{p} \sum_{q}\left[F\left(g, g^{+}\right) \frac{\overleftarrow{\partial}}{\partial g_{p}} \frac{\overleftarrow{\partial}}{\partial g_{q}}\right] \delta \tilde{g}_{q}(t) \delta \tilde{g}_{p}(t)+\cdots\right\}
\end{aligned}
$$

where we have used the Taylor expansion for $f\left(\tilde{g}_{p}(t+\delta t)\right)$ as in (156) with the notation as in $(65)$

$$
\delta \tilde{g}_{p}(t)=\tilde{g}_{p}(t+\delta t)-\tilde{g}_{p}(t)
$$

Note here that the $\delta \tilde{g}_{p}(t)$ can be any odd Grassmann functions and the Taylor expansion will still apply. If the $\delta \tilde{g}_{p}(t)$ are odd Grassmann functions then $\tilde{g}_{p}(t+\delta t)$ will behave as a Grassmann variable, as would be required. In obtaining the last result theorems in the previous Subsection relating the stochastic average of a sum to the sum of the stochastic averages have also been used. The quantities in the brackets $[\cdots]$ are functions of the $\tilde{g}_{p}(t)$ and do not depend on the $\delta \tilde{g}_{p}(t)$.

From the Ito stochastic equation in (63) the stochastic increment is given by

$$
\delta \tilde{g}_{p}(t)=C^{p}\left(\tilde{g}_{q}(t)\right) \delta t+\sum_{a} B_{a}^{p}\left(\tilde{g}_{q}(t)\right) \int_{t}^{t+\delta t} d t_{1} \Gamma_{a}\left(t_{1}\right)
$$

where the properties of the c-number Gaussian-Markoff random noise terms $\Gamma_{a}(t)(a=$ $1,2, \cdots, i(n))$ are set out in Eqs.(61) and (62). Overall then, the $\delta \tilde{g}_{p}(t)$ will be odd Grassmann functions, as required. At this stage the required number of Gaussian-Markoff random noise terms $\Gamma_{a}(t)$ is unspecified, but this will be some integer function $i(n)$ of the number of modes. 
With these results we can now obtain expressions for the stochastic averages. For the first order derivative terms we have

$$
\begin{aligned}
& \overline{\left\{\sum_{p}\left[F\left(g, g^{+}\right) \frac{\overleftarrow{\partial}}{\partial g_{p}}\right] \delta \tilde{g}_{p}(t)\right\}} \\
= & \frac{\left\{\sum_{p}\left[F \frac{\overleftarrow{\partial}}{\partial g_{p}}\right]\left[C^{p}\left(\tilde{g}_{q}(t)\right) \delta t+\sum_{a} B_{a}^{p}\left(\tilde{g}_{q}(t)\right) \int_{t}^{t+\delta t} d t_{1} \Gamma_{a}\left(t_{1}\right)\right]\right\}}{\overline{\sum_{p}\left[F \frac{\overleftarrow{\partial}}{\partial g_{p}}\right] \mathcal{C}^{p}\left(\tilde{g}_{q}(t)\right)} \delta t}+\overline{\sum_{P}\left[F \frac{\overleftarrow{\partial}}{\partial g_{p}}\right] \sum_{a} B_{a}^{p}\left(\tilde{g}_{q}(t)\right)} \overline{\int_{t}^{t+\delta t} d t_{1} \Gamma_{a}\left(t_{1}\right)} \\
= & \frac{\sum_{p}\left[F \frac{\overleftarrow{\partial}}{\partial g_{p}}\right] C^{p}\left(\tilde{g}_{q}(t)\right) \delta t}{}
\end{aligned}
$$

where the stochastic average rules for sums and products have been used, the non-correlation between the averages of functions of $\tilde{g}_{p}(t)$ at time $t$ and the $\Gamma$ are later times between $t$ to $t+\delta t$ is applied and the term involving $\overline{\Gamma_{a}\left(t_{1}\right)}$ is equal to zero from (61). Note that this term is proportional to $\delta t$.

For the second order derivative terms

$$
\begin{aligned}
& \overline{\left\{\frac{1}{2} \sum_{p, q}\left[F\left(g, g^{+}\right) \frac{\overleftarrow{\partial}}{\partial g_{p}} \frac{\overleftarrow{\partial}}{\partial g_{q}}\right] \delta \tilde{g}_{q}(t) \delta \tilde{g}_{p}(t)\right\}} \\
& =\left\{\begin{array}{c}
\frac{1}{2} \sum_{p, q}\left[F \frac{\overleftarrow{\partial}}{\partial g_{P}} \frac{\overleftarrow{\partial}}{\partial g_{q}}\right]\left[C^{q}\left(\tilde{g}_{r}(t)\right) \delta t+\sum_{a} B_{a}^{q}\left(\tilde{g}_{r}(t)\right) \int_{t}^{t+\delta t} d t_{1} \Gamma_{a}\left(t_{1}\right)\right] \\
\times\left[C^{p}\left(\tilde{g}_{r}(t)\right) \delta t+\sum_{b} B_{b}^{p}\left(\tilde{g}_{r}(t)\right) \int_{t}^{t+\delta t} d t_{2} \Gamma_{b}\left(t_{2}\right)\right]
\end{array}\right\} \\
& =\overline{\frac{1}{2} \sum_{p, q}\left[F \frac{\overleftarrow{\partial}}{\partial g_{p}} \frac{\overleftarrow{\partial}}{\partial g_{q}}\right]\left[C^{q}\left(\tilde{g}_{r}(t)\right) \delta t C^{p}\left(\tilde{g}_{r}(t)\right) \delta t\right]} \\
& +\frac{1}{2} \sum_{p, q}\left[F \frac{\overleftarrow{\partial}}{\partial g_{p}} \frac{\overleftarrow{\partial}}{\partial g_{q}}\right]\left[C^{q}\left(\tilde{g}_{r}(t)\right) \delta t \sum_{b} B_{b}^{p}\left(\tilde{g}_{r}(t)\right) \int_{t}^{t+\delta t} d t_{2} \Gamma_{b}\left(t_{2}\right)\right] \\
& +\overline{\frac{1}{2} \sum_{p, q}\left[F\left(g, g^{+}\right) \frac{\overleftarrow{\partial}}{\partial g_{p}} \frac{\overleftarrow{\partial}}{\partial g_{q}}\right]\left[\sum_{a} B_{a}^{q}\left(\tilde{g}_{r}(t)\right) \int_{t}^{t+\delta t} d t_{1} \Gamma_{a}\left(t_{1}\right) C^{p}\left(\tilde{g}_{r}(t)\right) \delta t\right]} \\
& +\overline{\frac{1}{2} \sum_{p, q}\left[F \frac{\overleftarrow{\partial}}{\partial g_{p}} \frac{\overleftarrow{\partial}}{\partial g_{q}}\right]\left[\sum_{a} B_{a}^{q}\left(\tilde{g}_{r}(t)\right) \int_{t}^{t+\delta t} d t_{1} \Gamma_{a}\left(t_{1}\right) \sum_{b} B_{b}^{p}\left(\tilde{g}_{r}(t)\right) \int_{t}^{t+\delta t} d t_{2} \Gamma_{b}\left(t_{2}\right)\right]}
\end{aligned}
$$


Using the result that the stochastic averages for the $\tilde{g}_{p}$ and the $\Gamma_{a}$ are uncorrelated we find that

$$
\begin{aligned}
& \overline{\left\{\frac{1}{2} \sum_{p, q}\left[F\left(g, g^{+}\right) \frac{\overleftarrow{\partial}}{\partial g_{p}} \frac{\overleftarrow{\partial}}{\partial g_{q}}\right] \delta \tilde{g}_{q}(t) \delta \tilde{g}_{p}(t)\right\}} \\
& =\overline{\frac{1}{2} \sum_{p, q}\left[F \frac{\overleftarrow{\partial}}{\partial g_{p}} \frac{\overleftarrow{\partial}}{\partial g_{q}}\right]\left[\mathcal{A}^{q}\left(\tilde{g}_{r}(t)\right) \mathcal{A}^{p}\left(\tilde{g}_{r}(t)\right)\right]} \delta t^{2} \\
& +\overline{\frac{1}{2} \sum_{p, q}\left[F \frac{\overleftarrow{\partial}}{\partial g_{p}} \frac{\overleftarrow{\partial}}{\partial g_{q}}\right]\left[\mathcal{A}^{q}\left(\tilde{g}_{r}(t)\right) \delta t \sum_{b} \mathcal{B}_{b}^{p}\left(\tilde{g}_{r}(t)\right)\right]} \overline{\int_{t}^{t+\delta t} d t_{2} \Gamma_{b}\left(t_{2}\right)} \\
& +\overline{\frac{1}{2} \sum_{p, q}\left[F \frac{\overleftarrow{\partial}}{\partial g_{p}} \frac{\overleftarrow{\partial}}{\partial g_{q}}\right]\left[\sum_{a} \mathcal{B}_{a}^{q}\left(\tilde{g}_{r}(t)\right) \mathcal{A}^{p}\left(\tilde{g}_{r}(t)\right) \delta t\right]} \overline{\int_{t}^{t+\delta t} d t_{1} \Gamma_{a}\left(t_{1}\right)} \\
& +\overline{\frac{1}{2} \sum_{p, q}\left[F \frac{\overleftarrow{\partial}}{\partial g_{p}} \frac{\overleftarrow{\partial}}{\partial g_{q}}\right]\left[\sum_{a} \mathcal{B}_{a}^{q}\left(\tilde{g}_{r}(t)\right) \sum_{b} \mathcal{B}_{b}^{p}\left(\tilde{g}_{r}(t)\right)\right]} \overline{\int_{t}^{t+\delta t} d t_{1} \Gamma_{a}\left(t_{1}\right) \int_{t}^{t+\delta t} d t_{2} \Gamma_{b}\left(t_{2}\right)}
\end{aligned}
$$

Now the terms involving a single $\Gamma$ have a zero stochastic average, whilst the terms with two $\Gamma$ give a stochastic average proportional to $\delta t$

$$
\begin{aligned}
\overline{\int_{t}^{t+\delta t} d t_{1} \Gamma_{a}\left(t_{1}\right) \int_{t}^{t+\delta t} d t_{2} \Gamma_{b}\left(t_{2}\right)} & =\int_{t}^{t+\delta t} d t_{1} \int_{t}^{t+\delta t} d t_{2} \overline{\Gamma_{a}\left(t_{1}\right) \Gamma_{b}\left(t_{2}\right)} \\
& =\int_{t}^{t+\delta t} d t_{1} \int_{t}^{t+\delta t} d t_{2} \delta_{a b} \delta\left(t_{1}-t_{2}\right) \\
& =\delta_{a b} \delta t
\end{aligned}
$$

so that correct to order $\delta t$ the second order derivative term is

$$
\begin{aligned}
& \left\{\frac{1}{2} \sum_{p, q}\left[F\left(g, g^{+}\right) \frac{\overleftarrow{\partial}}{\partial g_{p}} \frac{\overleftarrow{\partial}}{\partial g_{q}}\right] \delta \tilde{g}_{q}(t) \delta \tilde{g}_{p}(t)\right\} \\
& =\overline{\frac{1}{2} \sum_{p, q}\left[F\left(g, g^{+}\right) \frac{\overleftarrow{\partial}}{\partial g_{p}} \frac{\overleftarrow{\partial}}{\partial g_{q}}\right]\left[\sum_{a} B_{a}^{q}\left(g_{r}^{s}(t)\right) B_{a}^{p}\left(g_{r}^{s}(t)\right)\right]} \delta t \\
& =\overline{\frac{1}{2} \sum_{p, q}\left[F\left(g, g^{+}\right) \frac{\overleftarrow{\partial}}{\partial g_{p}} \frac{\overleftarrow{\partial}}{\partial g_{q}}\right]\left[\left[B\left(g_{r}^{s}(t)\right) B^{T}\left(g_{r}^{s}(t)\right)\right]_{q p}\right] \delta t} \text {. }
\end{aligned}
$$

The remaining terms give stochastic averages correct to order $\delta t^{2}$ or higher so that we have correct to first order in $\delta t$

$$
\begin{aligned}
& \overline{F\left(\tilde{g}(t+\delta t), \tilde{g}^{+}(t+\delta t)\right)}-\overline{F\left(\tilde{g}(t), \tilde{g}^{+}(t)\right)}=\overline{\sum_{p}\left[F\left(g, g^{+}\right) \frac{\overleftarrow{\partial}}{\partial g_{p}}\right] C^{p}\left(\tilde{g}_{q}(t)\right)} \delta t \\
& +\frac{1}{2} \sum_{p} \sum_{q}\left[F\left(g, g^{+}\right) \frac{\overleftarrow{\partial}}{\partial g_{p}} \frac{\overleftarrow{\partial}}{\partial g_{q}}\right]\left[B\left(\tilde{g}_{r}(t)\right) B^{T}\left(\tilde{g}_{r}(t)\right)\right]_{q p} \delta t
\end{aligned}
$$

or

$$
\frac{\frac{d}{d t} \overline{F\left(\tilde{g}(t), \tilde{g}^{+}(t)\right)}=\overline{\sum_{p}\left[F\left(g, g^{+}\right) \frac{\overleftarrow{\partial}}{\partial g_{p}}\right] C^{p}\left(\tilde{g}_{q}(t)\right)}}{+\frac{1}{2} \sum_{p} \sum_{q}\left[F\left(g, g^{+}\right) \frac{\overleftarrow{\partial}}{\partial g_{p}} \frac{\overleftarrow{\partial}}{\partial g_{q}}\right]\left[B\left(\tilde{g}_{r}(t)\right) B^{T}\left(\tilde{g}_{r}(t)\right)\right]_{q p}} .
$$




\section{Appendix H - Plimak et al Distribution Function}

In this Appendix the details involved in determining the $B$ distribution function at time $\delta t$ using the Plimak et al [23] ansatz are presented.

\subsubsection{Det $\beta(\delta t)$}

The determinent is given by

$$
\operatorname{Det} \beta=\sum_{k_{1}, k_{2}, \cdots, k_{2 n}} \beta_{1 k_{1}} \beta_{2 k_{2}} . \beta_{p k_{p}} . . \beta_{2 n k_{2 n}} \epsilon\left(k_{1}, k_{2}, \cdots, k_{p}, \cdots k_{2 n}\right)
$$

where $\epsilon\left(k_{1}, k_{2}, \cdots, k_{p}, \cdots k_{2 n}\right)=+1,-1$ if $k_{1}, k_{2}, \cdots, k_{p}, \cdots k_{2 n}$ is an even, odd permutation of $1,2, \cdots, 2 n$ and zero otherwise. Note that any particular matrix element $\beta_{p q}$ only appears once.

As

$$
\begin{aligned}
\left(\frac{\partial(\operatorname{Det} \beta)}{\partial \beta_{p q}}\right) & =\sum_{k_{1}, k_{2}, \cdots, k_{2 n}} \beta_{1 k_{1}} \beta_{2 k_{2}} . . \delta_{k_{p} q} . . \beta_{2 n k_{2 n}} \epsilon\left(k_{1}, k_{2}, \cdots, k_{p}, \cdots k_{2 n}\right) \\
& =\sum_{k_{1}, k_{2}, \cdots, k_{(p-1)} k_{(p+1)}, k_{2 n}} \beta_{1 k_{1}} \beta_{2 k_{2} . .} \beta_{(p-1) k_{(p-1)}} \beta_{(p+1) k_{(p+1)} .} . \beta_{2 n k_{2 n}} \epsilon\left(k_{1}, k_{2}, \cdots, q, \cdots k_{2 n}\right) \\
\left(\frac{\partial(\operatorname{Det} \beta)}{\partial \beta_{p q}}\right)_{0} & =\sum_{k_{1}, k_{2}, \cdots, k_{(p-1)} k_{(p+1)}, k_{2 n}} \delta_{1 k_{1}} \delta_{2 k_{2} .} . \delta_{(p-1) k_{(p-1)}} \delta_{(p+1) k_{(p+1)}} . . \delta_{2 n k_{2 n}} \epsilon\left(k_{1}, k_{2}, \cdots, q, \cdots k_{2 n}\right) \\
& =\delta_{p q}
\end{aligned}
$$

using $\beta(0)=E_{2 n}$.

Then with $\delta \beta(0)_{p q}=\beta(\delta t)_{p q}-\beta(0)_{p q}$

$$
\begin{aligned}
\operatorname{Det} \beta(\delta t) & =\operatorname{Det} \beta(0)+\sum_{p q}\left(\frac{\partial(\operatorname{Det} \beta)}{\partial \beta_{p q}}\right)_{0} \delta \beta(0)_{p q} \\
& =1+\sum_{p} \delta \beta(0)_{p p}
\end{aligned}
$$

\subsubsection{Stochastic Averages}

The determination of the various stochastic averages in Eq. (112) are as follows.

$$
\begin{aligned}
& \frac{\sum_{p} L_{p}^{p} \delta t \sum_{q}\left(B\left(g, g^{+}, 0\right) \frac{\overleftarrow{\partial}}{\partial g_{q}}\right) \delta \tilde{g}_{q}(0)}{=} \\
= & \sum_{p} L_{p}^{p} \delta t \sum_{q}\left(B\left(g, g^{+}, 0\right) \frac{\overleftarrow{\partial}}{\partial g_{q}}\right)\left(C^{q}\left(g_{q}\right) \delta t+\sum_{b} B_{b}^{q}\left(g_{q}\right) \delta \tilde{w}_{b}\left(0_{+}\right)\right) \\
= & 0+O(\delta t)^{2}
\end{aligned}
$$

and

$$
\begin{aligned}
& \overline{\sum_{p} L_{p}^{p} \delta t \frac{1}{2} \sum_{r q}\left(B\left(g, g^{+}, 0\right) \frac{\overleftarrow{\partial}}{\partial g_{q}} \frac{\overleftarrow{\partial}}{\partial g_{r}}\right) \delta \tilde{g}_{r}(0) \delta \tilde{g}_{q}(0)} \\
= & \sum_{p} L_{p}^{p} \delta t \frac{1}{2} \sum_{r q}\left(B\left(g, g^{+}, 0\right) \frac{\overleftarrow{\partial}}{\partial g_{q}} \frac{\overleftarrow{\partial}}{\partial g_{r}}\right)\left(C^{r}\left(g_{q}\right) \delta t+\sum_{a} B_{a}^{r}\left(g_{q}\right) \delta \tilde{w}_{a}\left(0_{+}\right)\right)\left(C^{q}\left(g_{q}\right) \delta t+\sum_{b} B_{b}^{q}\left(g_{q}\right) \delta \tilde{w}_{b}\left(0_{+}\right)\right.
\end{aligned}
$$


and

$$
\begin{aligned}
& \overline{\sum_{p a} K_{p, a}^{p} \delta \tilde{w}_{a}\left(0_{+}\right) \sum_{q}\left(B\left(g, g^{+}, 0\right) \frac{\overleftarrow{\partial}}{\partial g_{q}}\right) \delta \tilde{g}_{q}(0)} \\
= & \overline{\sum_{p a} K_{p, a}^{p} \delta \tilde{w}_{a}\left(0_{+}\right) \sum_{q}\left(B\left(g, g^{+}, 0\right) \frac{\overleftarrow{\partial}}{\partial g_{q}}\right)\left(C^{q}\left(g_{q}\right) \delta t+\sum_{b} B_{b}^{q}\left(g_{q}\right) \delta \tilde{w}_{b}\left(0_{+}\right)\right)} \\
= & -\sum_{p a} K_{p, a}^{p} B_{a}^{q}\left(g_{q}\right) \sum_{q}\left(B\left(g, g^{+}, 0\right) \frac{\overleftarrow{\partial}}{\partial g_{q}}\right) \delta t \\
= & +\sum_{p q}\left(B\left(g, g^{+}, 0\right) \frac{\overleftarrow{\partial}}{\partial g_{q}}\right)\left(\sum_{a} K_{p, a}^{p} B_{a}^{q}\left(g_{q}\right)\right) \delta t
\end{aligned}
$$

and

$$
\begin{aligned}
& \overline{\sum_{p a} K_{p, a}^{p} \delta \tilde{w}_{a}\left(0_{+}\right) \frac{1}{2} \sum_{r q}\left(B\left(g, g^{+}, 0\right) \frac{\overleftarrow{\partial}}{\partial g_{q}} \frac{\overleftarrow{\partial}}{\partial g_{r}}\right) \delta \tilde{g}_{r}(0) \delta \tilde{g}_{q}(0)} \\
= & \sum_{p a} K_{p, a}^{p} \delta \tilde{w}_{a}\left(0_{+}\right) \frac{1}{2} \sum_{r q}\left(B\left(g, g^{+}, 0\right) \frac{\overleftarrow{\partial}}{\partial g_{q}} \frac{\overleftarrow{\partial}}{\partial g_{r}}\right)\left(C^{r}\left(g_{q}\right) \delta t+\sum_{c} B_{c}^{r}\left(g_{q}\right) \delta \tilde{w}_{c}\left(0_{+}\right)\right)\left(C^{q}\left(g_{q}\right) \delta t+\sum_{b} B_{b}^{q}\left(g_{q}\right)\right. \\
= & 0+O(\delta t)^{2}
\end{aligned}
$$

\subsubsection{Comparisons}

Here we evaluate some of the expressions in (114) and (116).

Firstly, using (76)

$$
\begin{aligned}
D_{q p} \frac{\overleftarrow{\partial}}{\partial g_{q}} & =\sum_{a} \sum_{r} K_{r, a}^{q} g_{r} \sum_{s} K_{s, a}^{p} g_{s} \frac{\overleftarrow{\partial}}{\partial g_{q}} \\
& =\sum_{a} \sum_{r, s} K_{r, a}^{q} K_{s, a}^{p}\left(g_{r} \delta_{s, q}-\delta_{r, q} g_{s}\right) \\
& =\sum_{a} \sum_{r} K_{r, a}^{q} K_{q, a}^{p} g_{r}-\sum_{a} \sum_{s} K_{q, a}^{q} K_{s, a}^{p} g_{s} \\
& =\sum_{a} B_{a}^{q} K_{q, a}^{p}-\sum_{a} K_{q, a}^{q} B_{a}^{p} \\
\sum_{q} D_{q p} \frac{\overleftarrow{\partial}}{\partial g_{q}} & =\sum_{q, a} B_{a}^{q} K_{q, a}^{p}-\sum_{q, a} K_{q, a}^{q} B_{a}^{p}
\end{aligned}
$$

using (75).

Secondly,

$$
\begin{aligned}
D_{p q} \frac{\overleftarrow{\partial}}{\partial g_{q}} \frac{\overleftarrow{\partial}}{\partial g_{p}} & =-D_{q p} \frac{\overleftarrow{\partial}}{\partial g_{q}} \frac{\overleftarrow{\partial}}{\partial g_{p}} \\
& =-\sum_{a} \sum_{r} K_{r, a}^{q} K_{q, a}^{p} g_{r} \frac{\overleftarrow{\partial}}{\partial g_{p}}+\sum_{a} \sum_{s} K_{q, a}^{q} K_{s, a}^{p} g_{s} \frac{\overleftarrow{\partial}}{\partial g_{p}} \\
& =-\sum_{a} K_{p, a}^{q} K_{q, a}^{p}+\sum_{a} K_{q, a}^{q} K_{p, a}^{p} \\
\sum_{p, q} D_{p q} \frac{\overleftarrow{\partial}}{\partial g_{q}} \frac{\overleftarrow{\partial}}{\partial g_{p}} & =-\sum_{p q a} K_{p, a}^{q} K_{q, a}^{p}+\sum_{p q a} K_{q, a}^{q} K_{p, a}^{p}
\end{aligned}
$$


Thirdly, using (78)

$$
\begin{aligned}
A_{p} \frac{\overleftarrow{\partial}}{\partial g_{p}} & =-\sum_{r} L_{r}^{p} g_{r} \frac{\overleftarrow{\partial}}{\partial g_{p}} \\
& =-L_{p}^{p} \\
-\sum_{p}\left(A_{p} \frac{\overleftarrow{\partial}}{\partial g_{p}}\right) & =\sum_{p} L_{p}^{p}
\end{aligned}
$$

